\title{
OXAPROZIN-LOADED LIPID NANOPARTICLES TOWARDS OVERCOMING NSAIDS SIDE-EFFECTS
}

JOSÉ PLÁCIDO LOPES DE ARAÚJO

DISSERTAÇ̃̃O DE MESTRADO APRESENTADA

Ã FACULDADE DE ENGENHARIA DA UNIVERSIDADE DO PORTO EM

BIOENGENHARIA - BIOTECNOLOGIA MOLECULAR 
(․ José Araújo, 2014 
Faculdade de Engenharia da Universidade do Porto Instituto de Ciências Biomédicas Abel Salazar
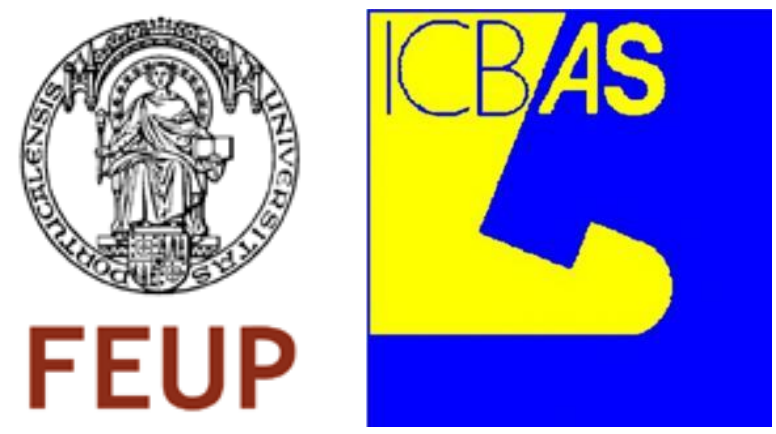

\section{Oxaprozin-loaded Lipid Nanoparticles towards overcoming NSAIDs side-effects}

José Plácido Lopes de Araújo

Master Thesis for the degree in Master of Science in BioEngineering Specialization in Molecular Biotechnology

Supervisors: Prof. Dr. Salette Reis

Dr. Cláudia Nunes

July 2014 
(․ José Araújo, 2014 


\section{Abstract}

The inflammatory process is the innate immune response for the presence of pathogens, toxic molecules, tissue injuries or any other harmful conditions. The inflammation process is characterized for redness, pain, swelling, heat and disturbance of function and comprises inducers, sensors, mediators and effectors components from cellular and humoral origin. Macrophages are one of the most important cells in the inflammatory process. Macrophages actively phagocyte particles with sizes superiors to $200 \mathrm{~nm}$ and express folate receptor making them of great interest for passive and active targeting strategies. Non-Steroidal AntiInflammatory Drugs, like oxaprozin, are one of the most used drugs prescribed for these conditions, however these drugs have adverse side effects, namely at the level of the gastric mucosa, that must be avoided and pharmacokinetic properties that need to be improved and for these purpose many delivery systems arise. Lipid Nanoparticles allow an effective drug packaging and targeted delivery, improving drug's pharmacokinetics and pharmacodynamics properties and avoiding some of their side effects. In this work, two formulations containing oxaprozin were developed: nanostructured lipid carriers with and without folate functionalization. Folate functionalization was obtained by the addition of a synthesised DSPE-PEG 2000 -FA conjugate. These formulations had low polydispersity (approximately 0.150 ) with mean diameters around $285 \mathrm{~nm}$ and zeta potential superior to $-40 \mathrm{mV}$. The elevated encapsulation efficiency of the particles (superior to 95\%) along with the high amount of used oxaprozin lead to a high loading capacity (close to 9\%). The formulations avoided the oxaprozin release in simulated gastric fluid promoting its release on simulated intestinal fluid, physiologic and inflammatory medium, remaining only less than $10 \%$ of the oxaprozin entrapped on the lipid carrier matrix. MTT and LSH assays revealed that the formulations only seemed to present cytotoxicity in Caco-2 cells for oxaprozin concentrations superiors to 100 $\mu \mathrm{M}$. Permeability studies in Caco-2 cell lines shown that oxaprozin encapsulation did not interfere with oxaprozin permeability, having the final formulations an apparent permeability around $0.8 \times 10^{-5} \mathrm{~cm} \cdot \mathrm{s}^{-1}$ in simulated intestinal fluid and about $1.45 \times 10^{-5} \mathrm{~cm} \cdot \mathrm{s}^{-1} \mathrm{PBS}$.

Keywords- Inflammation, Oxaprozin, Nanostructured Lipid Carriers, Nanoparticles Characterization, Folate Functionalization, In vitro Release Study, MTT assay, LDH assay, Caco-2 Permeability study. 
-This page was intentionally left blank- 


\section{Acknowledgments}

I express my deepest and sincere gratitude to Professor Salette Reis for once again giving me the opportunity to work with his research group at the Faculty of Pharmacy and for hall the guidance, encouragement and concern.

I am deeply grateful to Dr. Cláudia Nunes for these two years of guidance and care! I am also deeply sorry for being so annoying sometimes, but that was my "job". I want you to know that if you are the guilty for all the things that go wrong on the lab and all the "open doors" you also are the guilty for everything that had gone well, for everything that I have learn and all the doors that may have been open to my areas of knowledge and who knows my future.

I would also like to thank all the girls and couple of boys of the lab for all the good moments, help and kind words along the time and for making the job more fun and relaxed. I let a special thank for Nini and Virgínia for the special help and work on some experimental points.

To my friends "Tecos" for the moments of support, all the friendship, talks, laughs and "milkshake" moments with no sense at all but with all the sense in the world. But that is how we are...

To my parents, brother and grandmother, without you this work have been impossible. Thank you for all the support, education, values and the effort that you have made for making my dreams come true. And I want to thank Daniela for being always there with her unconditional and endless love, continuous support and encouraging smile! 
-This page was intentionally left blank- 


\section{Table of Contents}

Abstract..........................................................................

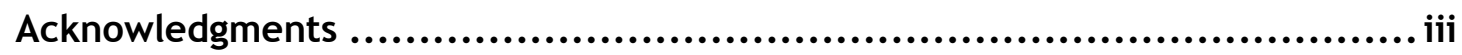

Table of Contents.............................................................v



List of Tables ............................................................. viii

Glossary ....................................................................... ix

Chapter 1 .................................................................... 1

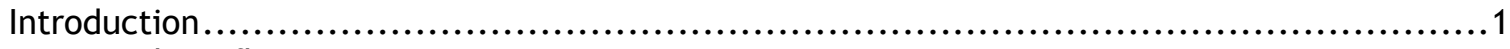

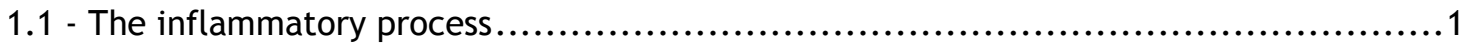

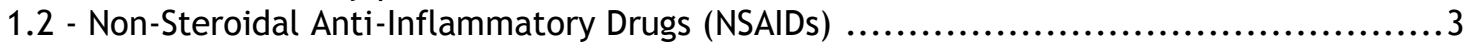

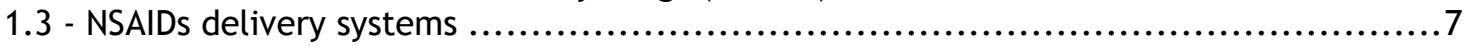

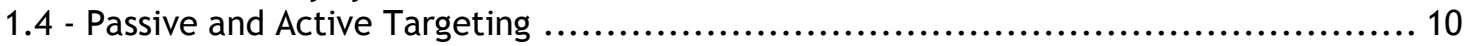

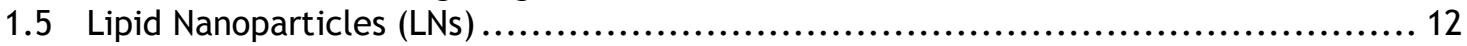

1.6 Oral Administration.................................................................... 15

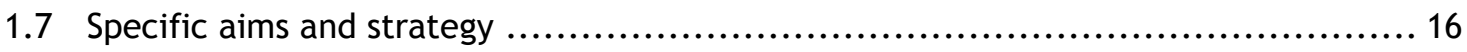

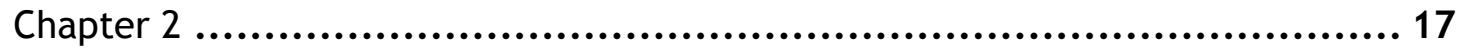



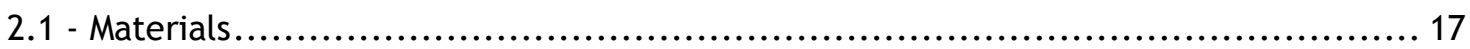

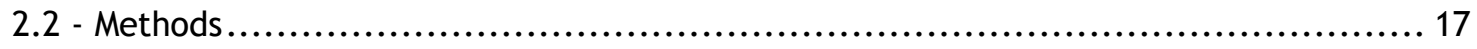

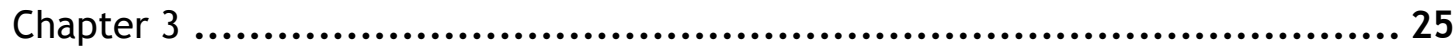

Results and Discussion ................................................................ 25

3.1 - Choice of solid lipid for improved drug loading ................................... 25

3.2 - Lipid nanoparticles synthesis and optimization ...................................... 26

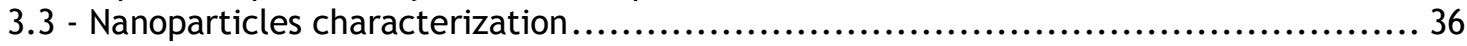

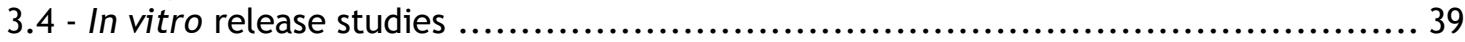

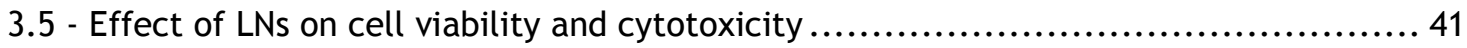

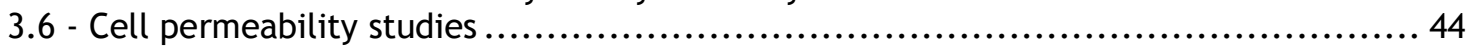

Chapter 4 .................................................................... 47

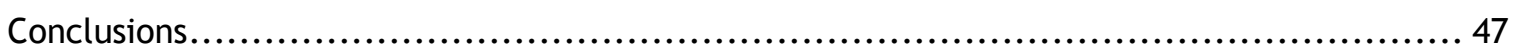




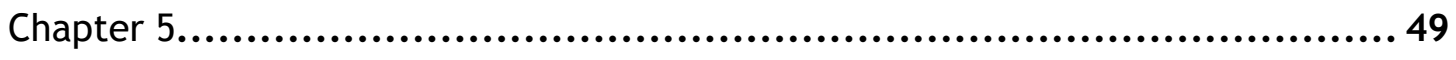



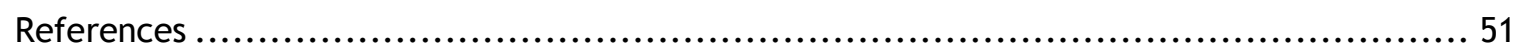




\section{List of Figures}

Figure 1: Overview of the inflammatory process and its mediators ${ }^{[15]}$

Figure 2: NSAIDs mechanism of action and non-selective (traditional) and COX-2 selective inhibited (coxibs) pathways

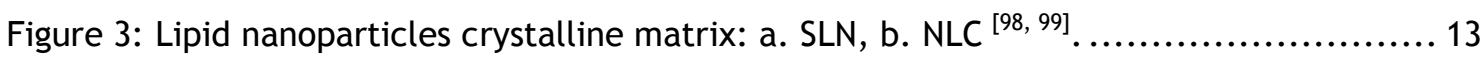

Figure 4: Lipid nanoparticles synthesised by emulsification-sonication ....................... 36

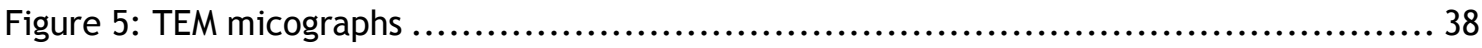

Figure 6: UV-Vis analysis of the functionalization process in Functionalized NLCs placebo.... 39

Figure 7: UV-Vis analysis of the functionalization process on Functionalized NLCs +

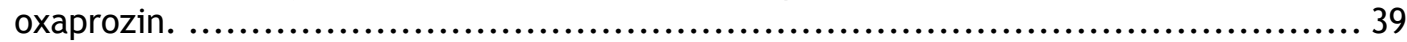

Figure 8: Oxaprozin release in gastric, intestinal and physiologic media. .................... 40

Figure 9: Oxaprozin release in gastric, intestinal and inflammatory media $\ldots . . . \ldots \ldots . . . . . .40$

Figure 10: Oxaprozin release in gastric, intestinal, physiological and inflammatory media. .. 41

Figure 11: General morphology of confluent Caco-2 cells by inverted microscope observation ..................................................................... 42

Figure 13: Caco-2 cell cytotoxicity assessed by LDH assay as a function of the different formulations and concentrations tested $(5,10,50,100,500,1000 \mu \mathrm{M})$.

Figure 12: Caco-2 cell viability assessed by MTT assay as a function of the different formulations and concentrations tested $(5,10,50,100,500,1000 \mu \mathrm{M}) . \ldots \ldots \ldots \ldots \ldots . . .43$

Figure 14: Monolayer formation on the Transwell inserts along time.

Figure 16: Oxaprozin permeability along the time and final apparent permeability (Table) for free oxaprozin and functionalized and non-functionalized oxaprozin NLCs in PBS. .. 45

Figure 15: Oxaprozin permeability along the time and final apparent permeability (Table) for free oxaprozin and functionalized and non functionalized oxaprozin NLCs in FaSSIF. 


\section{List of Tables}

Table 1: Structure and properties of NSAIDs....................................................

Table 2: Lipids phase transition temperature and qualitative oxaprozin dissolution

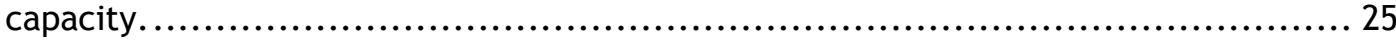

Table 3: List of the synthesised LNs during the optimization process. ....................... 27

Table 4: Physicochemical properties of the developed LNs. Mean effective diameter, polydispersity, zeta potential, encapsulation efficiency and loading capacity of placebo and oxaprozin non-functionalized and folate functionalized LNs............... 37 
-This page was intentionally left blank- 


\section{Glossary}

\begin{tabular}{|c|c|}
\hline AA & Arachidonic Acid \\
\hline ANOVA & Analysis of Variance \\
\hline APMA & $\mathrm{N}$-(3-aminopropyl) methacrylamide hydrochloride \\
\hline CLRs & C-type Lectin Receptors \\
\hline $\operatorname{cox}$ & Cyclooxygenase \\
\hline DAMPs & Danger-Associated Molecular Patterns \\
\hline DCC & Dicyclohexylcarbodiimide \\
\hline DCU & Dicylcohexylurea \\
\hline DMEM & Dulbecco's Modified Eagle Medium \\
\hline DSPE-PEG-FA & Disteroylphosphatidylethanolamine-poly(ethylene glycol)-folic \\
\hline DSPE-PEG-NH 2 & $\begin{array}{l}\text { (1,2-distearoyl-sn-glycero-3-phosphoethanolamine- } \mathrm{N} \text { - } \\
\text { [amino(polyethylene glycol)-2000] (ammonium salt)) }\end{array}$ \\
\hline DLS & Dynamic Light Scattering \\
\hline DMSO & Dimethyl Sulfoxide \\
\hline DOCA & Deoxycholic Acid \\
\hline EDTA & Ethylenediaminetetraacetic acid \\
\hline $\mathrm{EE}$ & Encapsulation Efficiency \\
\hline ELS & Electrophoretic Light Scattering \\
\hline EPR & Enhanced Permeability and Retention \\
\hline FA & Folic Acid \\
\hline FaSSGF & Fasted State Simulated Gastric Fluid \\
\hline FaSSIF & Fasted State Simulated Intestinal Fluid \\
\hline FBS & Fetal Bovine Serum \\
\hline FDA & Food and Drug Administration \\
\hline $\mathrm{FR}$ & Folate Receptor \\
\hline GI & Gastro-Intestinal \\
\hline GRAS & Generally Recognized As Safe \\
\hline HBSS & Hanks' Balanced Salt Solution \\
\hline $\mathrm{HPH}$ & High Pressure Homogenisation \\
\hline ICAM-1 & Intercellular Adhesion molecule-1 \\
\hline IL & Interleukin \\
\hline LBP & Lipopolysaccharide Binding Protein \\
\hline LC & Loading Capacity \\
\hline LDH & Lactase Dyhydrogenase \\
\hline Leu & Leucine \\
\hline LM & Lipid Microsphere \\
\hline LNs & Lipid Nanoparticles \\
\hline$M$ cells & Microfold Cells \\
\hline MBP & Mannose Binding Protein \\
\hline MTT & Methylthiazolyldiphenyl-tetratozium Bromide \\
\hline NIPAAm & $\mathrm{N}$-isopropyl acrylamide \\
\hline
\end{tabular}




$\begin{array}{ll}\text { NLCs } & \text { Nanostructured Lipid Carriers } \\ \text { NLRs } & \text { NOD-like Receptors } \\ \text { NHS } & \text { N-hydroxysuccinimide } \\ \text { NHS-FA } & \text { N-hydroxysuccinimide ester of folic acid } \\ \text { NMR } & \text { Nuclear Magnetic Resonance } \\ \text { NSAIDs } & \text { Non-Steroidal Anti-Inflammatory Drugs } \\ \text { Oxa } & \text { Oxaprozin } \\ \text { Papp } & \text { Apparent permeability coefficient } \\ \text { PAMAM } & \text { Polyamidoamine } \\ \text { PAMPs } & \text { Pathogen-Associated Molecular Patterns } \\ \text { PBS } & \text { Phosphate Buffered Saline } \\ \text { PEG } & \text { Poly(ethylene glycol) } \\ \text { Pen Strep } & \text { Penicillin-Streptomycin } \\ \text { PEO-b-PDPA } & \text { Poly(ethylene oxide)-b-poly[2-(diisopropylamino)-ethyl methacrylate] } \\ \text { PEO-PBLA } & \text { Poly(ethylene oxide)-poly(B-benzyl L-aspartate) } \\ \text { pHEMA } & \text { Poly(hydroxyethyl methacrylate) } \\ \text { PLGA } & \text { Poly(lactide-co-glycolide) } \\ \text { PLGA-PEG } & \text { Poly(lactide-co-glycolide-ethylene-glycol) } \\ \text { PGs } & \text { Prostaglandins } \\ \text { PPO } & \text { Polypropylene Oxide } \\ \text { PPRs } & \text { Pattern-Recognition Receptors } \\ \text { RGD } & \text { Arg-Gly-Asp } \\ \text { RNS } & \text { Reactive Nitrogen Species } \\ \text { ROS } & \text { Reactive Oxygen Species } \\ \text { SD } & \text { Standard Deviation } \\ \text { SLNs } & \text { Solid Lipid Nanoparticles } \\ \text { TEA } & \text { Triethylamine } \\ \text { TEER } & \text { Transepithelial Electrical Resistance } \\ \text { TEM } & \text { Transmission electron microscopy } \\ \text { TGL-B } & \text { Transforming Growth Factor B } \\ \text { TLRs } & \text { Toll-Like Receptors } \\ \text { TNF } & \text { Tumor Necrosis Factor } \\ \text { TX-100 } & \text { Triton X-100 } \\ \text { VP } & \text { W/o/w }\end{array}$





\section{Chapter 1}

\section{Introduction}

Chronic inflammation conditions are on the base of several diseases, reason why the development of effective therapies in the treatment of these conditions is of extreme importance [2-5].

Non-Steroidal Anti-Inflammatory Drugs (NSAIDs) are one of the most used drugs prescribed for these conditions however, like every drug they present adverse side effects [6-9].

In order to surpass these adverse effects, specialized delivery systems may be of extreme importance and may hold the answer for an effective anti-inflammatory treatment of such conditions.

\section{1 - The inflammatory process}

The presence of pathogens, toxic molecules, tissue injuries or any other harmful conditions in an organism will origin a defensive response in order to restore the cellular homeostasis [2]. The human body comprise three distinct defence systems that include the anatomic and physiologic barriers, the innate immunity and the adaptative immunity [10].

The innate immune response is characterized by an inflammatory process that is traditionally defined by four symptoms related to the immune system's response. These wellknown symptoms are redness, pain, swelling and heat (in Latin rubor, dolor, tumor, and calor respectively) $[2,3,5,11,12]$. Another symptom is disturbance of function (in Latin functio laesa), which was posteriorly added to the previous four $[3,5,12]$. These symptoms have origin in vascular and cellular changes promoted by inflammatory mediators that are produced by inflammatory and tissue cells or by metabolization of their extracellular precursors [12].

The inflammatory process includes cellular elements: monocytes, macrophages, neutrophils, dendritic cells, and natural killer cells (whose main functions are to phagocyte and kill the inflammatory agents and antigen-presentation), eosinophils, basophils and mast cells (responsible for the production of the inflammatory mediators) and humoral elements: complement, lysozime, lactoferrin and antimicrobial peptides [5, 10, 11, 13, 14]. The components of the inflammatory process can be characterized as inducers, sensors, mediators and effectors and this process is summarized in the Figure 1.

The origin of the inducers determines their classification. Exogenous inducers can be divided in microbial inducers: Pathogen-Associated Molecular Patterns (PAMPs) and virulence factors; and non-microbial inducers which include toxic compounds, foreign bodies, irritants and allergens. Endogenous inducers are generally signals produced by tissues in response to stress, damage or malfunctioning, designated Danger-Associated Molecular Patterns (DAMPs) $[2,14]$. 


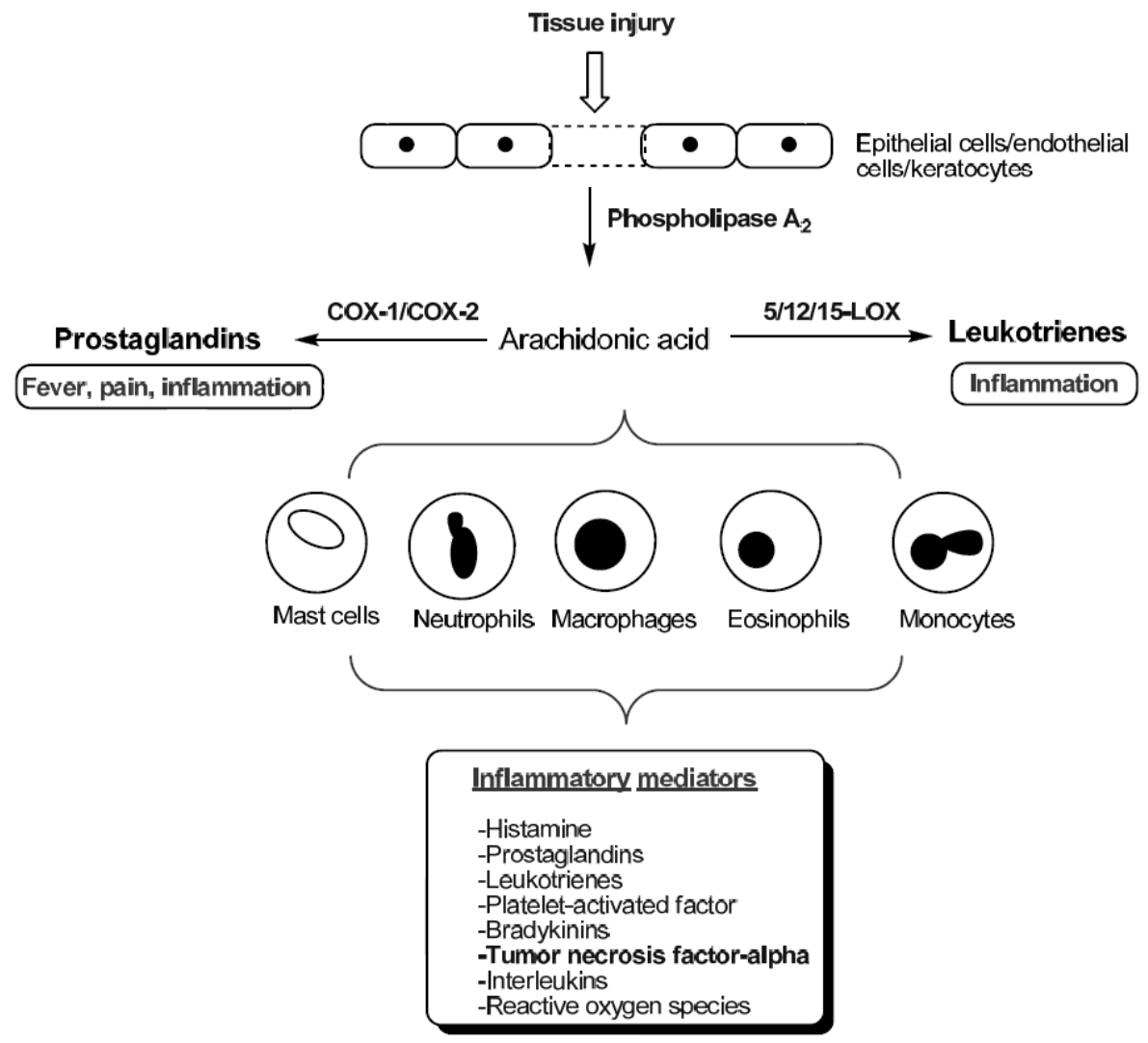

Figure 1: Overview of the inflammatory process and its mediators ${ }^{[15]}$.

Sensor components comprise specific membrane receptors like Pattern-Recognition Receptors (PPRs) that recognise PAMPs and DAMPs, Toll-Like Receptors (TLRs), C-type Lectin Receptors (CLRs), NOD-like Receptors (NLRs), Mannose Binding Protein (MBP) and Lipopolysaccharide Binding Protein (LBP) [2, 10, 13, 14].The interaction between inducers and sensors leads to the production of mediator compounds derived from plasma proteins or secreted by cells. Mediator compounds can be classified as [2-5, 11, 13-15]:

a) Vasoactive amines like histamine and serotonin that act on the vasculature leading to vasodilation or vasoconstriction, affecting also the vascular permeability;

b) Vasoactive peptides like substance $\mathrm{P}$, kinins, thrombin or plasmin that also can cause vasodilation and increased vascular permeability;

c) Complement Proteins that act by opsonising pathogens surface, i.e. by coating the surface making the pathogens more easily phagocytised. Moreover, they may also create a membrane-attack complex that generate a pore on the pathogen's lipidic bilayer membrane;

d) Proteolytic enzymes that act in order to allow leukocyte migration degrading the extra cellular matrix and the basement-membrane proteins;

e) Cytokines that induce local vasodilation, increased blood flow and vascular permeability leading also to leukocytes activation. Cytokines promote as well the influx of cell to the inflammation site; 
f) Chemokines responsible for the chemotaxis that direct the leukocyte traffic towards the inflammation site, acting as directional signals and also by the recruitment of leukocytes;

g) Reactive Oxygen Species (ROS) as superoxide, hydroxyl radicals, singlet oxygen, oxygen halides, hydrogen peroxide and Reactive Nitrogen Species (RNS) that interact with lipids, proteins, nucleic acids and other molecules of the pathogens leading to its dead;

h) Lipid mediators like eicosanoids and platelet-activating factors. Arachidonic acid (AA) metabolism by cyclooxygenases (COX-1 and COX-2) leads to the formation of eicosanoids, which in turn generate prostaglandins (PGs) that cause vasodilation and are responsible for fever, pain, fatigue, sleepiness, anorexia and social withdrawal, since they act on the central nervous system. The platelet-activating factors, also produced by this pathway, activate several process related to leukocytes activation and vascular regulation.

These inflammatory mediators induce the effectors, generally cells, creating the inflammatory response and may also take action as amplifiers of the inflammatory process acting as chemoattractants $[3,14]$.

Because all the inflammatory components possess numerous elements, different inflammatory pathways can be created so each inflammatory process is unique [5].

The inflammatory process begins within moments from the inducer exposure which explain why, for some, the innate immunity is the most important type of immunity [2, 10, 13].

All these processes are extremely beneficial for the host, however they must be highly regulated since some of them are not able to discriminate between microbial and host cells leading to tissue damages [14]. In order to control the inflammatory process, proinflammatory cytokines are produced such as Tumor Necrosis Factor (TNF) and Interleukin 1 and 6 (IL-1 and IL-6) [2, 4]. Negative feedback mechanisms include the inhibition of proinflammatory signalling cascades, loss of mediator's receptors, activation of regulatory cells and the production of anti-inflammatory cytokines such as IL-10 and IL-13, Transforming Growth Factor $B($ TGF- $B$ ) and glucocorticoids [2-4, 14]. A balance between pro-inflammatory and anti-inflammatory factors is essential to create an appropriate and effective inflammatory response.

When the pathogen or the damaged tissue has been completely removed, the inflammation process should stop allowing the tissue repair and healing. So, when the regulatory process or the acute inflammatory response fails, the process turns into a chronic inflammatory condition. These chronic inflammatory conditions, in turn, lead to host tissue lesions and are implicated in many diseases such as rheumatoid arthritis, atherosclerosis, type 2 diabetes, neurodegenerative diseases asthma, inflammatory bowel diseases, cancer and obesity [2-5].

\section{2 - Non-Steroidal Anti-Inflammatory Drugs (NSAIDs)}

Because of the major role that COX plays both in the acute and chronic inflammatory process, the use of drugs that successfully block this pathway is of extreme importance. Since Non-Steroidal Anti-Inflammatory Drugs have the capability to block COX, even at $\mathrm{mM}$ 
concentrations, they have become an indispensable choice in some chronic inflammatory diseases and in pain management $[16,17]$.

\subsubsection{Interplay between mechanism of action and therapeutic effects}

NSAIDs have analgesic, antipyretic, and anti-inflammatory effects being effective drugs in reducing swelling, redness and pain in inflammatory conditions and also general fever and headache. NSAIDs are one of the most effective and prescribed drugs used to treat pain and inflammatory conditions in patients suffering from osteoarthritis, rheumatoid arthritis, gout, low back pain, soft tissue pain and chronic pain [6-9]. NSAIDs act by blocking the cyclooxygenases in a competitive way, inhibiting therefore prostanoids synthesis $[7,12,18]$.

COX exists at least in two isoforms: COX-1 and COX-2 [12, 19, 20]. COX-1 and COX-2 are heme-containing bifunctional proteins that convert AA into PG-G2 by a cyclooxygenation reaction and this into $\mathrm{PG}-\mathrm{H} 2$ by a peroxidase reaction. The activity of tissue-specific synthases and isomerases convert PG-H2 into different prostanoids like PG-E2, PG-D2, PGF2a, prostacyclin (PG-12) and thromboxane A2 (TX-A2) [12, 19-21]. COX-1 is constitutively expressed being present in almost every tissue and leads to the production of PGs that help in the control of vascular homeostasis, platelet, gastric and renal activity and also protects the gastric mucosa. COX-2 is an inducible cyclooxygenase, active in cells that line blood vessels and that catalysis the production of pro-inflammatory PGs in response to inflammatory factors $[6,12,19]$. Thus, NSAIDs analgesic and anti-inflammatory effects are a consequence of their ability to block COX-2 activity.

NSAIDs are characterized in two distinct classes according to their ability to selectively block COX-2 and this is given by the ratio of the IC50 for COX-1 and COX-2 with in vitro studies [22-24]:

a) Non-selective COX inhibitors: block both COX-1 and COX-2 in a non-selective manner. They traditionally possess a carboxylic acid $(-\mathrm{COOH})$ as a functional group and represent the most prescribed NSAIDs for fever, pain and inflammation treatment;

b) Cox-2 selective inhibitors: selectively block COX-2. Generally they possess a diarylheterocyclic ring as template and less adverse effects.

The mechanism of action and the pathways that are blocked by both NSAIDs classes are summarized in Figure 2.

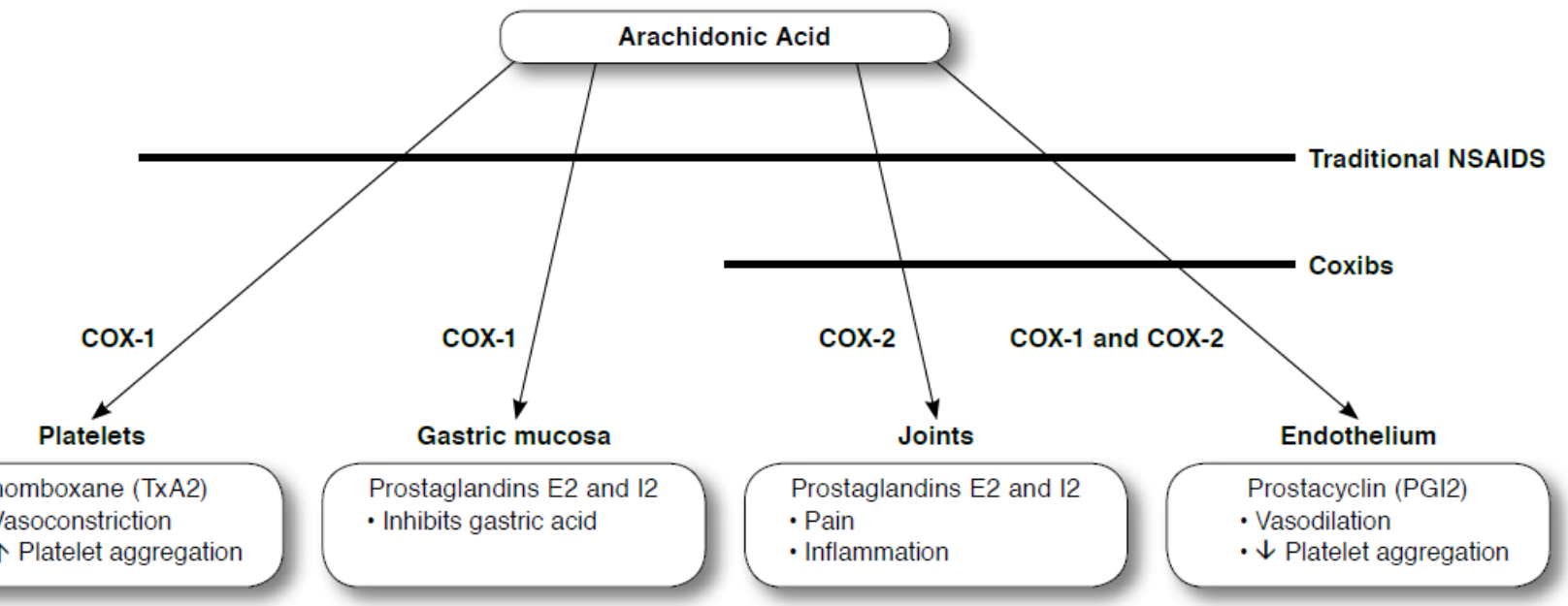

Figure 2: NSAIDs mechanism of action and non-selective (traditional) and COX-2 selective inhibited (coxibs) pathways ${ }^{[1]}$. 


\subsubsection{Interplay between mechanism of action and side effects}

Despite its beneficial effects NSAIDs, present side effects such as toxicity in the gastrointestinal tract, liver, brain, cardiovascular and renal systems, skin, platelet aggregation and blood pressure [6, 7, 16-18, 25-28].

Bloating, cramping, pain, diarrhoea, constipation, acid reflux, erosins, duodenal and gastric ulcers, perforations, obstructions and bleeding are among the NSAIDs effects on the gastrointestinal tract $[6,8,16,18,29]$. The carboxylic acid that generally makes part of the NSAIDs chemical structure leads to gastrointestinal irritation when orally administrated [16, 30]. Besides that, because non-selective COX inhibitors block COX-1 activity, they prevent mucosal cytoprotective PGs formation enhancing gastrointestinal toxicity $[8,9,19,20]$.

NSAIDs effects in renal homeostasis lead to sodium retention, peripheral oedema, weight gain, high blood pressure, congestive heart failure, hyperkaelemia, and acute renal failure. PGs produced for both COX have an important role on renal vasculature regulation and on the electrolyte dynamics, decreasing $\mathrm{Na}+$ reabsorption, so blocking its production leads to deregulation on these processes and ultimately to the effects previously mentioned $[6,16$, 19, 31].

COX-2 selective inhibitors toxicity is mostly related to cardiovascular complications like hypertension and increased risk of myocardial infarction and atrial fibrillation. These effects occur due to the absence of important PGs that control platelet-wall interactions, vasoconstriction, platelet aggregation, cholesterol accumulation and that antagonize the effects of hypertensive drugs $[6,7,16,19,23,24]$.

\subsubsection{Chemical classification}

NSAIDs are a very heterogeneous group of molecules with different molecular structures, being the majority organic acids with low water solubility [18, 30, 32-34]. NSAIDs capability to interact with COX-1 and COX-2 is directly related to its chemical properties allowing the carboxylic acid the interaction with COX-1 and having lipophilic drugs a more strong interaction with COX-2 [35]. So, non-selective COX inhibitors generally have carboxylic acids or ketoenolic acids in their constitution while COX-2 selective inhibitors have sulphonamide functional groups on a diarylheterocyclic ring. COX-2 selective inhibitors have been created in order to possess low gastrointestinal adverse effects, reason why their moieties confer them high values of pKa and avoid their interaction with COX-1 [30]. Some NSAIDs properties are summarized on Table1. 
Table 1: Structure and properties of NSAIDs ${ }^{[32,36]}$.

\begin{tabular}{|c|c|c|c|c|}
\hline Drug & Structure & pKa & Mol wt & $\log P$ \\
\hline \multicolumn{5}{|c|}{ Non-selective COX inhibitors } \\
\hline Aspirin & & 3.5 & 180.2 & -1.1 \\
\hline Diclofenac & & 4.2 & 296.1 & 4.5 \\
\hline Flurbiprofen & & 4.22 & 244.3 & 4.2 \\
\hline Ibuprofen & & 4.4 & 206.3 & 4.0 \\
\hline Indomethacin & & 4.2 & 357.8 & 4.5 \\
\hline Ketoprofen & & 4.5 & 254 & ---- \\
\hline Naproxen & & 4.2 & 230.3 & 3.2 \\
\hline Oxaprozin & & 4.3 & 293 & 4.8 \\
\hline COX-2 selecti & & & & \\
\hline Celecoxib & & 10.7 & 381.4 & 3.9 \\
\hline
\end{tabular}

\subsubsection{Oxaprozin}

Oxaprozin (4,5-diphenyl-2-oxazolepropionic acid; Wy-21,743; CAS-21256-18-8) (Table 1) is a non-selective COX inhibitor NSAID with effective anti-inflammatory, analgesic and antipyretic effects. These therapeutic activities derive from its capability to inhibit COX-2 [37-42]. Oxaprozin has an aliphatic propionic acid function attached to the oxazole as side chain, however contrarily to other propionic acid compounds this doesn't possess a chiral centre [40, 42].

Oxaprozin (Oxa) has a molecular weight of $293 \mathrm{~g} / \mathrm{mol}$ and presents as a lipophilic white powder, with low water solubility and being slightly soluble in alcohol. It's a weak acid with pKa around 4.3 with high gastric mucosa tolerance comparatively to other NSAIDs of the same class. That also leads to a slow rate of absorption on the gastric mucosa at stomach $\mathrm{pH}$. Oxaprozin highly binds to plasma proteins $(99.9 \%)$ with high affinity what leads to a long 
plasma elimination with a half-life of 50 to 60 hours. This elevated duration of action allows the administration of a single oxaprozin dose per day of 600 to $1800 \mathrm{mg}$ [32, 39, 40, 42, 43].

Oxaprozin like the other NSAIDs and every drug have possible side effects that include stomach pain, constipation, diarrhoea, gas, heartburn, nausea, vomiting and dizziness. More serious side effects include heart attack, stroke, high blood pressure, heart failure (from body swelling due to liquid retention), kidney problems (including kidney failure), stomach and intestine bleeding and ulcers, anaemia, life-threatening skin reactions, life-threatening allergic reactions, liver problems (including liver failure) and asthma attacks (in asthma patients) [44].

\section{3 - NSAIDs delivery systems}

In order to reduce the adverse side effects of NSAIDs, improve NSAIDs pharmacokinetic properties and to promote a sustained, controlled and targeted drug delivery, several delivery systems have been created in the last years. Next, we will shortly describe some relevant examples of published works in this field.

To the date, most of the developed NSAIDs delivery systems are based on polymeric micro/nanospheres. Many NSAIDs have been formulated into microspheres using biodegradable and non-biodegradable polymers and various methods for oral, ocular, parenteral and topical applications

Calvo et al. prepared three independent nanocarriers made out of poly- $\varepsilon$-caprolactone loaded with indomethacin, a nanoparticle, a nanocapsule and a nanoemulsion and also a microparticle made out of the same polymer. They verified that these nanosystems had a threefold higher corneal epithelium penetration when compared to a commercial solution, without causing membrane damages. In contrast, the microparticle wasn't able to increase indomethacin ocular bioavailability [45, 46].

Jayaprakash et al. designed a copolymeric nanoparticle composed by $\mathrm{N}$ isopropylacrylamide, vinyl pyrrolidone and acrylic acid loaded with ketorolac. These nanoparticles were produced in order to increase this NSAID bioavailability. This formulation showed, in vitro, a twofold NSAID permeation and a higher anti-inflammatory activity compared with its aqueous suspension [47].

By a quasi-emulsion solvent technique, Pignatello et al. have been able to produce a polymeric nanoparticle suspension of Eudragit RS $100 \AA$ loaded with ibuprofen. These nanoparticles allowed a controlled delivery of ibuprofen and an improvement on its ocular availability by increasing its corneal retention and a gradual and prolonged drug release due to its positively charged surface that helps corneal adhesion without ocular tissue toxicity [48].

The same authors have also produced by the same technique a polymeric nanoparticle suspension of Eudragit RS $100 \otimes$ and RL100® loaded with flurbiprofen, in order to also improve its ocular availability. Once again this cationic polymeric nanoparticle suspension indicated a controlled drug delivery and high efficiency when compared to the aqueous drug solution [49].

Eudragit RS100® NSAID loaded nanoparticle were also a matter of study for Adibkia et al.. They loaded these nanoparticles with piroxicam and used them to control inflammatory symptoms in rabbits, concluding that the nanoparticles inhibited the inflammation more successfully than the aqueous suspension of the drug [50]. 
Polylactic and polyglycolic acid have been used in order to produce nanoparticles loaded with flurbiprofen by Vega et al. and these authors concluded that these nanoparticles were a promising ocular delivery system of drugs due to its excellent ocular tolerance and protection against sodium arachidonate induced inflammation [51].

Badawi et al. loaded indomethacin into chitosan nanoparticles and chitosan nanoemulsion creating positively charged nanocarriers with good corneal affinity and a gradual and longterm (up to 24h) drug release to both external and internal ocular tissues [52].

Contreras-García et al. grafted the co-polymers of $\mathrm{N}$-isopropyl acrylamide (NIPAAm) and $\mathrm{N}$-(3-aminopropyl) methacrylamide hydrochloride (APMA) into a polypropylene film and then loaded the film with NSAIDs in order to analyse their cytocompatibility and frictional properties and as well to evaluate its capability to load and release, for long periods, therapeutic doses of NSAIDs. They concluded that this grafts cold be used in the design of products for biomedical devices in order to promote a controlled NSAIDs release [53].

Alginate microparticles can be used in order to store NSAIDs and deliver NSAIDs, however these microparticles can be destabilised in acidic environments. So in order to create gastroresistent alginate- $\mathrm{OCH}$ microparticles, Čalija et al. used an anionic copolymer, Eudragit $\circledast$ L100-55, to stabilize the alginate-OCH microparticles in acidic conditions, leading to a decreased drug release at gastric $\mathrm{pH}$ and sustained drug release at intestinal $\mathrm{pH}$. These microparticles may be used for oral NSAIDs delivery due its $\mathrm{pH}$ sensitive capacities [54].

Polymeric micelles of poly(ethylene oxide)-poly(B-benzyl L-aspartate) (PEO-PBLA) were used to encapsulate indomethacin by La et al.. The drug released from the micelles was highly $\mathrm{pH}$ dependent with rapid release profiles for $\mathrm{pH}$ around 7.4 [55].

Giacomelli et al. used poly(2-(dialkylamino)ethyl methacrylate) as micellar core of poly(ethylene oxide)-b-poly[2-(diisopropylamino)-ethyl methacrylate] (PEO-b-PDPA) or poly(glycerolmonomethacrylate)-b-PDPA (PG2MA-b-PDPA) micelles. These micelles allowed a more stable indomethacin encapsulation and prolonged drug retention profiles due to ionic and hydrophobic interactions [56].

Marques et al. formulated nanosized micelles, with different amphiphilic chitosan based polymers in their constitution, loaded with ibuprofen and functionalized with deoxycholic acid (DOCA) and leucine (Leu) moieties. These self-assembled nanoparticles successfully encapsulated the NSAID and delivered it into tumor cells. The anti-tumoral effect of this nanosystem is comparable with that of the traditionally used chemotherapeutics [57].

Roullin et al. prepared biodegradable nanospheres using poly(lactide-co-glycolide) (PLGA). These non toxic nanoparticles showed good NSAIDs encapsulation and local controlled NSAIDs release for up to $48 \mathrm{~h}$. These nanospheres were found to be suitable for oral administration and for prosthesis surface coating [58].

Bédouet et al. created a hydrogel biodegradable microspheres prepared by copolymerization of a methacrylic derivative of ibuprofen with oligo(ethyleneglycol)methacrylate and poly(lactide-co-glycolide-ethylene-glycol) (PLGA-PEG) dimethacrylate as degradable crosslinker. These microspheres were able to prolong the drug release time avoiding the initial burst release, keeping the drug anti-inflammatory activity. So they are suitable for drug delivery by intra-articular injection [59].

Koç et al. designed a class of polyamidoamine (PAMAM) dendrimers for high NSAIDs performance and solubility. For that they introduced a polypropylene oxide as core of the PAMAM (PPO@PAMAM) dendrimers and loaded the dendrimers with ketoprofen, ibuprofen and 
diflunisal. These formulations augmented NSAIDs solubility, increasing this solubility with the dendrimer concentration and generation in an almost proportional way [60].

Hasegawa et al. reported the creation of a NSAID micellar prodrug using hydrophilic poly(ethylene glycol) (PEG) and a polyacrylamide derivative of ibuprofen as amphiphilic diblock copolymers. Ibuprofen release occurs due to chemical and enzymatic hydrolysis of the micelles and takes place over several days to weeks. These micelles increased ibuprofen solubility and were able to efficiently inhibit two cancer cell lines proliferation [61].

In order to promote corneal residence of indomethacin and consequently augment its ocular availability Balasubramaniam et al. created a Gelrit gellan gum in situ gelling system for topical drop administration. This system was therapeutically effective in rabbits and allowed an in vitro release for up to 8h [62].

Bycopolymerization of poly(hydroxyethyl methacrylate) (PHEMA) with low APMA or 4vinyl-pyridine (VP) proportions Andrade-Vivero et al. created a hydrogel with high NSAIDs loading capacity. Due to this hydrogel properties such as high biocompatibility, thermal stability, acidic and alkaline hydrolysis resistance and easily adaptable mechanical properties, it can be used in oral controlled NSAIDs delivery systems, implants for drug delivery, soft contact lenses (in order to prevent bacterial colonization) or other biomedical devices [63].

In order to provide NSAIDs oral administration Corrente et al. formulated a diclofenac loaded $\mathrm{pH}$ sensitive hydrogel using Scl-CM-300 from the scleroglucan polymers class. This hydrogel revealed mucoadhesive properties, affinity to aqueous media, $\mathrm{pH}$ sensitive behaviour and biocompatible properties, providing a prolonged drug release time, avoiding the ulcerogenic effects of this NSAID. The authors suggested that this formulation could be used for oral delivery of ulcerogenic NSAIDs or for colon targeting [64].

Saxena et al. created an emulgel (gel emulsion) with Gelucire 39/01 as lipid phase and low viscosity sodium alginate aqueous solution as aqueous phase. These emulgel was loaded with piroxicam for oral administration. These gel jellifies in situ, which prevents the NSAID direct contact with the gastric mucosa avoiding the NSAID side effects while providing sustain drug release [65].

Del Gaudio et al. created almost spherical alginate-based aerogels loaded with ketoprofen with porous texture similar to the related hydrogels. The system responded rapidly to $\mathrm{pH}$ changes dissolving the drug. The interesting properties of the aerogel make it suitable for the fast delivery of NSAIDs in the upper gastrointestinal tract which could be useful for NSAID fast delivery for analgesic effects in postoperative pain, dental surgery, renal and uretal acute colic [66].

Sousa et al. produced, by an alumina template assisted synthesis, silica nanotubes with $\mathrm{Si}-\mathrm{O}-\mathrm{H}$ chemical bounds in the inner surface used to create a polycationic surface. The naproxen loaded nanotubes allowed a pH controlled sustainable drug release. The authors suggested the external functionalization of the nanotubes with folate in order to target the $\mathrm{pH}$ sensitive delivery systems for the inflamed tissue [67].

Consola et al. described the formation on a cationic vesicle assembled with an amino sugar surfactant and NSAIDs in an aqueous solution. The result was a water soluble cationic vesicle that when used for dermal administration of the NSAIDs showed an improved antiinflammatory activity and prolonged time of NSAIDs residence due to a slower skin diffusion of the system. Besides that, this formulation assembles spontaneously, use biocompatible surfactants and an aqueous vehicle [68]. 
A lipo-preparation made out of lecithin coated lipid grains with a soybean oil core allowed the formation, by Ohmukai et al., of a lipid microsphere (LM) loaded with flurbiprofen axetil dissolved on the soybean oil. This LM reduced the adverse effects of flurbiprofen prompting its analgesic effects. It can be used for intravenous administration especially on postoperative pain since it lacks central nervous system adverse reactions [69].

Joshi et al. compared a commercial alcoholic solution of valdecoxib with a Nanostructured Lipid Carriers (NLC) loaded with the same drug. In vitro studies revealed a sustained drug release from the NLC contrarily to what happens with the commercial solution, this drug release goes for up to $24 \mathrm{~h}$ in a in vivo study [70].

The same authors also compared in vivo a NLC based gel loaded with colecoxib with a micellar gel with the same composition. The NLC had a better behaviour than the micellar gel having a better penetration and sustained drug release leading to oedema inhibition for up to 24h [71].

Han et al. formulated a flurbiprofen loaded NLC and compared its physical stability and permeation with a NSAID buffer solution. They concluded that the NLC was stable for the study period and that the skin permeation was improved in relation to the NSAID solution making this a suitable system for NSAIDs dermal delivery system [72].

Attama et al. designed Solid Lipid Nanoparticles (SLN), with phospholipids in its constitution, loaded with sodium diclofenac. The technique used by the authors allowed high drug encapsulation and the SLN were able to successfully release the drug in a sustained manner and this way these nanoparticles revealed a good performance for ocular application [73].

The production of cationic liposomes loaded with diclofenac sodium to be used as a aqueous eye drop formulation was reported by Sun et al.. This nanosystem allowed the NSAID pre-corneal retention with a $211 \%$ improvement in aqueous humour concentrations [74].

Tsukamoto et al. created submicron-sized liposomes coated with chitosan loaded with sodium bromfenac, with high encapsulation efficiency, for ocular drug delivery. Varying lipid concentrations, inner water phase, initial drug liposomal concentration and surface properties the authors were able to control drug release time in vitro for up to hours [75].

Despite all these efforts to improve NSAIDs features and NSAIDs based treatments only few NSAIDs delivery systems are on the line to get Food and Drug Administration (FDA) approval [1].

\section{4 - Passive and Active Targeting}

Targeting systems are designed in order to direct delivery systems to its specific target like an organ, tissue or a cell. So this will, consequently, increase drug concentration in the site of action and improve the selectivity of the delivery systems. Targeting systems can be classified as passive and active [76].

\subsubsection{Passive targeting}

This type of targeting can be achieved without the incorporation of specific targeting moieties on the drug system surface [76].

In the presence of an inflammatory process, vasculature permeability and dilation may suffer alterations due to the expression of several mediators. So, in inflammation sites the permeability of the vasculature is increased allowing the penetration to the interstitial site of 
particles that normally wouldn't be able to pass through the vasculature [77, 78]. This process is generally known as the Enhanced Permeability and Retention (EPR) effect [77, 79]. This EPR effect can be used for passive targeting of inflammation sites. For this, the drug system size is an important factor and must be between approximately $50 \mathrm{kDa}$ and $200 \mathrm{~nm}$ in order to pass through the vessels by the EPR effect and also avoiding glomerular filtration [79]. The circulation time of these systems is also important since long circulation times will increase its accumulation on the inflammation site [79].

Taking advantage of EPR effect, Srinath et al. produced indomethacin loaded liposomes. The indomethacin was entrapped in the bilayer of the liposome due to its amphiphilic characteristics. The mean size of these liposomes was $100 \mathrm{~nm}$ what allowed them to pass the vessel walls into the inflammation site which can justify why these liposomes where able to successfully reduce joint inflammation in rats while a two times higher dose of free indomethacin showed only a limited effect [80].

Similarly, Palakurthi et al. encapsulated indomethacin in the oily core of lipid nanospheres composed by soybean oil, phosphatidylcholine and cholesterol. These nanospheres had mean sizes of $150 \mathrm{~nm}$ and some of these nanospheres were coated with PEG in order to achieve long circulation times. The in vitro clearance of the free drug was reduced when encapsulated being this reduction superior for the PEGylated nanospheres. The PEGylated nanospheres had also better drug targeting efficiency and high drug accumulation in the rat arthritic paw [81].

Particles that are too big to be cleared renally will be fagocyted in the liver and spleen, however the therapeutic consequences of this are more complex when we talk of macrophage passive targeting since particles with sizes superior to $200 \mathrm{~nm}$ are taken up by macrophages $[77,79]$.

Although the inflammatory pathways may differ with the inflammation inducer, the macrophages are an essential and common component of these processes which make them a good target [76, 77].

The $\mathrm{pH}$ of an inflammation site environment is significantly lower than the surrounding environment being around 6.4 for inflammation sites and 7.4 for healthy tissues [82]. So, this factor can be used in the creation of $\mathrm{pH}$ sensitive targeting systems that will only release the drug in low $\mathrm{pH}$.

Other factors such as particle charge, inflammation site temperature (generally hyperthermia is associated with inflammation), elevated elastase levels and high enzymatic activity may also be used for passive targeting of inflammation [76, 77]. Kim et al. created poly(ethylene oxide)-poly(propylene oxide)-poly(ethylene oxide)/poly( $\varepsilon$-caprolactone) amphiphilic block copolymeric nanospheres loaded with indomethacin. These nanospheres showed reversible size changes depending on the temperature as well the indomethacin release behaviour. Indomethacin cytotoxicity was also evaluated and the authors concluded that the nanospheres diminished indomethacin cell damages [83].

\subsubsection{Active targeting}

Active targeting is achieved by particles surface modifications with targeting moieties which leads to specific interactions between the drug system and the target site through specific ligand-receptor interactions being the drug specifically delivered to the site of action $[76,77]$. 
Activated phagocytes tend to phagocyte PEG-coated vesicles at inflammation sites. This may happen due to the presence of high levels of phospholipase and its high catalytic activity toward these PEG-coated vesicles [84]. Besides that, PEG prolongs the circulation time of the particles enhancing also its retention by passive targeting due to EPR effect [84].

Due to the vascular alterations, endothelial cells enhance the expression of numerous types of adhesions molecules like selectins, integrins and immunoglobulins that are essential in the regulation of the inflammatory process and the migration of inflammatory cell from the vessel to the inflammation site [78]. One example of these molecules is the extensively studied Intercellular Adhesion molecule-1 (ICAM-1) which participates in the trafficking of inflammatory cells and is considered one of the most important adhesion factors for leukocyte recruitment to inflamed sites. So ICAM- 1 is a prominent target for active targeting systems $[78,85]$.

In inflammatory conditions the oxygen and nutrients deficiency induces the angiogenesis, i.e. the formation of new blood vessels. This process is carried by the influence of both vascular endothelial cells and monocyte-derived cells (which includes the macrophages), so these cells are good candidates for active targeting [79, 86, 87]. In the angiogenesis process several cell-surface receptors, adhesion proteins and growth factors are evolved making of them potential targets for therapeutic intervention [78].

Although several molecules may be recognized as potential targets the most used targets for active targeting purposes are the Folate Receptor (FR) and the avB3 integrin [88-90].

FR is overexpressed in activated macrophages and tumor cells. There are two isoforms of the FR: FR- $\alpha$ (expressed in cancer cells) and FR-B (expressed in activated macrophages) [91, 92]. This way there is a potential in the targeting of activated macrophages using $F R-B$ as target. It's possible to perform this targeting conjugating folate or folic acid on the drug system surface [79, 93]. Chandrasekar et al., for example, created a folate-dendrimer conjugate loaded with indomethacin for specific drug delivery to inflammatory sites. In order to achieve it, they coupled folic acid to PAMAM dendrimers of four generation. When compared to non-conjugated polymers, its in vitro release rate decreased and the drug's halflife, residence time and targeting efficiency augmented [94].

avB3 integrin is a surface receptor expressed in several cells such as endothelial cells and macrophages and is only expressed in the presence of a neovascularisation process which makes this receptor a target for inflammation. It is possible to target avB3 integrin using a cyclic peptide composing by an Arg-Gly-Asp (RGD) sequence as ligand [90, 95, 96].

Another method that can be used for active targeting it's the guidance of the drug system through the application of an electric field. Timko et al. developed magnetite polymer nanospheres loaded with indomethacin. These particles showed good NSAID encapsulation and capability to respond to external magnetic fields showing that this system can be used for magnetic drug targeting [97].

\subsection{Lipid Nanoparticles (LNs)}

Lipid nanoparticles are nanosized particles with a solid lipidic matrix. Its constituents are excipients recognised as safe, being Generally Recognized As Safe (GRAS) substances [98, 99]. Lipid nanoparticles can be mainly divided into two types: Solid Lipid Nanoparticles (SLN) and Nanostructured Lipid Carriers (NLC) [100-103]. 


\subsubsection{Solid Lipid Nanoparticles (SLN)}

SLN are biocompatible and biodegradable particles prepared using solid lipids at room and body temperature and stabilized by surfactants. These particles sizes go from approximately 40 to up $1000 \mathrm{~nm}$ and they can be used for target-specific controlled drug delivery by several administration routes. SLN have a highly ordered crystalline structure and the drugs are loaded between the fatty acid chains, the lipid layers and crystal imperfection [98, 103].

SLN can be used to incorporate both lipophilic and hydrophilic drugs, due to its high water content, increasing the drug stability and being able to protect photosensitive, moisture sensitive and chemically labile molecules from degradation by environmental factors. The avoidance to use organic solvents and the use of GRAS elements is also of extreme importance and a great advantage concerning the no toxicity of these particles. The production methods are easy and inexpensive and can easily be scaled up to industrial production so as the sterilization method [98, 100, 102-105].

Because of their highly organized structure (Figure 3a.) these particles aren't able to bear high drug amounts, besides that during storage polymorphic transitions may occur since the crystalline structure tends to rearrange its imperfections leading to drug expulsion changing the drug release profile. During the storage may also occur gelation of the dispersion and particle growth [98, 100, 101, 105].

\subsubsection{Nanostructured Lipid Carriers (NLC)}

NLC have been introduced in order to surpass the low payload and drug expulsion of the SLN. These nanoparticles have a solid lipid matrix at room temperature and are constituted by solid lipids and liquid lipids (in small amounts) at the room and body temperature in order to create imperfections in the crystalline structure (Figure 3b.) and larger distances between the fatty acid chains of the glycerides of the different used lipids [98, 100-103, 105, 106].

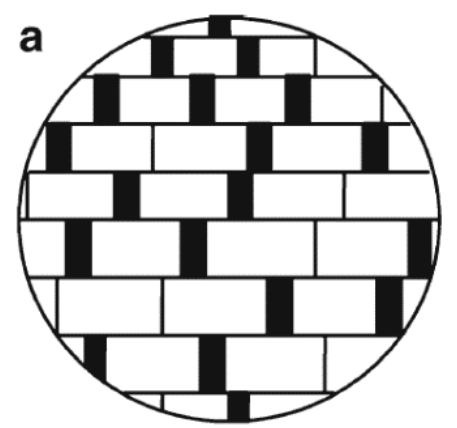

Lipid

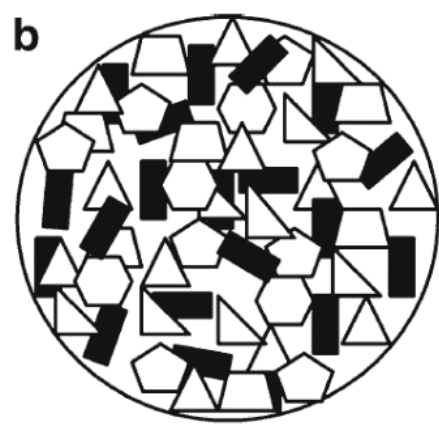

Drug molecule

Figure 3: Lipid nanoparticles crystalline matrix: a. SLN, b. NLC ${ }^{[98,99]}$.

NLC are able to increase the chemical properties of the stacked drugs and to provide a targeted and controlled drug release. Because of their imperfect crystalline structure, NLC are able to load higher amounts of drugs, besides that the water content of NLC is inferior to that of SLN. Drug expulsion during storage time and gelation of the dispersion are reduced. These nanoparticles allow the formation of final doses forms such as tablets and capsules and NLC production can also be adapted to large scale production [100-102, 106]. 


\subsubsection{LNs preparation}

Various methods can be used to produce LNs. Following, these methods will be shortly described [98, 100, 104]:

a) High Pressure Homogenisation (HPH): This method can be performed both at high (hot $\mathrm{HPH}$ ) and low (cold HPH) temperatures:

1. Hot HPH: Both lipid and drug are melted and then combined with an aqueous surfactant solution at the same temperature. By high speed stirring of this solution a hot pre-emulsion is formed that can then be processed in a temperature controlled high pressure homogeniser forming a nanoemulsion. Cooling down the nanoemulsion to room temperature leads to its recrystallization forming LNs;

2. Cold HPH: The lipid and the drug are also melted together and then are rapidly placed in liquid nitrogen forming solid lipid microparticles. A presuspension is then formed by high speed stirring of the particles in a cold aqueous surfactant solution. The pre-suspension is then homogenised at room temperature leading to LNs formation;

b) Microemulsion: The solid lipids are melted and then the drug is dissolved in them. A surfactant-cosurfactant solution at a temperature above the melting temperature of the solid lipids is then added with mild agitation in order to obtain a microemulsion. This microemulsion is then dispersed in cold water with mild agitation forming ultrafine nanoemulsion droplets that immediately crystallize to form LNs. The excess water can then be removed;

c) Solvent emulsification-evaporation: The lipids are dissolved in a water-immiscible organic solvent and then, under continuous stirring, emulsified in an aqueous phase containing surfactants. During the emulsification the organic solvent evaporates and the lipid precipitates forming LNs;

d) Solvent diffusion: This technique uses water-miscible organic solvents that are saturated with water in order to ensure initial thermodynamic equilibrium of both liquids. The transient oil-in-water emulsion is passed into water under continuous stirring, leading to solidification of dispersed phase and LNs formation due to diffusion of the organic solvent;

e) Solvent injection: Lipids are dissolved in a water-miscible solvent or water-miscible solvent mixture and then, using an injection needle, the solution is quickly injected into an aqueous surfactant solution;

f) Double emulsion: w/o/w (water in oil in water) double emulsion method is based on solvent emulsion-evaporation method. This method uses a stabilizer during hydrophilic drug encapsulation in the internal water phase of a w/o/w double emulsion, avoiding drug partitioning to the external water phase during solvent evaporation.

g) High speed stirring and/or ultra-sonication: Lipid microparticles are produced by spray congealing, these particles are then used to produce lipid nanopellets by high speed stirring or sonication.

h) Emulsification-Sonification: The lipids are melted at temperatures slightly above their melting point and then the drug is dissolved in the lipids. An aqueous surfactant solution at the same temperature is then added and the solution is homogeneously 
dispersed by a high shear homogenization device and the obtained emulsion is then subjected to ultrasonication forming LNs.

\subsection{Oral Administration}

Oral administration is the most used route of administration, being more than $60 \%$ of the drugs in the market, administered via oral administration [107]. Oral administration convenience and patient compliance are two major factors that lead to an increased therapeutic efficacy of orally administered drugs and that help to explain its importance and preference [108, 109]. Moreover, oral formulations production is cheaper because they do not need to be manufactured under sterile conditions [110].

Molecules and particles absorption in the gastro-intestinal (GI) tract occur mainly by four mechanisms that enable their passage by the cell membrane namely: paracellular, trancelullar, carrier-mediated and receptor-mediated transports. Absorption through these pathways vary with different physical characteristics like pH stability, ionization constants, hydrophobicity and molecular weight and also with site characteristics as enzyme activity, $\mathrm{pH}$, mucosa constitution, residence time and surface area [108, 111]. Paracellular transport comprises substance passage through the existing intercellular spaces between epithelial cells, primarily the tight junctions due a passive transport by diffusion [108]. Transcellular transport, in which the substance is taken by intestinal epithelial cells at the cell apical membrane by endocytosis transport through the cell and released at the basolateral cell membrane [112]. Carrier-mediated transport, an active transport process that requires energy. Substance transport across the cell requires its specific binding to the carriers on the membrane. [108, 113]. Receptor-mediated transport by cell invagination after a ligandreceptor bound leading to vesicle formation and endocytosis [108].

Since one of the $\mathrm{Gl}$ tract main functions is to break-down and destroy substances, it is to expect that oral formulations must deal with numerous hurdles [109].

Throughout the $\mathrm{Gl}$ tract one can observe a high $\mathrm{pH}$ range that goes from the extremely acidic gastric environment with $\mathrm{pH}$ from 1 to 3 , to duodenal $\mathrm{pH}$ from 6.0 to 6.5 , and large intestine pH from 5.5 to 7 [114]. Throughout the Gl tract different enzymes are synthesised and released being the $\mathrm{Gl}$ tract filled with numerous proteolytic enzymes that are able to degrade all kind of substances [115].

Despite the mucus layer protective effect on the epithelial cells against this $\mathrm{Gl}$ harsh environment, mucus layer can also have a major role on substances absorption in the GI tract. GI tract mucus layer entrap particles leading to its rapid clearance both due to quick mucus cell turnover and through an immune system attack [108, 109].

$\mathrm{Gl}$ epithelium is highly impermeable having tight junctions an important role since they only allow the passage of small slightly charged particles with sizes inferior to $2 \mathrm{~nm}$ [116118].

Despite all these barriers, oral administration encloses several advantages like a large surface augmenting substances uptake; the mucus, despites its barrier effect, also helps to enlarge substances retention time and its trancellular migration and the presence of microfold cells ( $M$ cells) that are generally less protected by mucus and that actively phagocytise bigger substances into systemic circulation [109, 118]. 
Nanoparticles drug encapsulation may help to protect the drugs against the harsh Gl environment ( $\mathrm{pH}$ and enzymes) and also increase mucoadhesion and retention in the $\mathrm{Gl}$ tract leading to an improved drug uptake [109].

\subsection{Specific aims and strategy}

In this context the main objective of this work, is to develop an innovative oxaprozin delivery system, based on lipid nanoparticles, with the main purpose of overcoming the most deleterious side effects of this drug - gastric disorders. Thereby, the main steps included during the first semester, literary research, state of the art and work plan elaboration. The second part, the research work, was fulfilled during the second semester at the Department of Chemical Sciences at the Faculty of Pharmacy, University of Porto.

Research work consisted first on the production of oxaprozin loaded lipid that embodies the selection of an appropriate lipid mixture according to the solubility of the drug, the optimization of the production method and of the lipid phase constitution according to the encapsulation efficiency, loading capacity, particles diameter and zeta potential. The second step was surface functionalization with Folic Acid (FA), this step involved the synthesis of a DSPE-PEG $2000-F A$ conjugate using DSPE-PEG $2000-\mathrm{NH}_{2}$ and folic acid. Third step was the characterization of the developed drug delivery systems by experiments of particle size and zeta potential using dynamic light scattering (DLS) and electrophoretic light scattering (ELS) respectively, encapsulation efficiency, loading capacity and functionalization analysis using UV-Vis spectroscopy and in vitro release studies at physiological conditions. The final step was the validation of the developed drug delivery systems by cell viability and cytotoxicity studies in Caco-2 cell line using Methylthiazolyldiphenyl-tetratozium Bromide (MTT) and Lactase Dyhydrogenase (LDH) and permeability studies in Caco-2 cell lines by UV-Vis spectroscopy quantification.

This approach could improve the NSAIDs use by reducing its serious side-effects, promoting its controlled release and drug action in inflamed regions. 


\section{Chapter 2}

\section{Materials and Methods}

\section{1 - Materials}

The lipids Apifil, Cetyl Palmitate, Compritol HD5 A, Gelucire 33/01 Lipocire CM, Precirol Ato 5 and Superpolystrate were a kind gift from Gattefossé (Gattefossé, France) and the lipids Imwitor 308, Softisan 100, Witepsol E76, Witepsol E85, Witepsol H32, Witepsol S51 and Witepsol 558 were gently offered by Sasol (Sasol, Germany). Miglyol 812 was purchased from

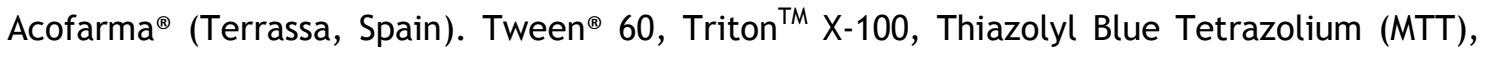
Trypan Blue powder, dimethyl sulfoxide $\geq 99.9 \%$, oxaprozin (4,5-diphenyl-2-oxazolepropanoic acid; Daypro), acetic acid $\geq 99.8 \%$, potassium phosphate monobasic, folic acid, triethylamine, dicyclohexylcarbodiimide, N-hydroxysuccinimide and Dulbecco's Phosphate Buffered Saline pH 7.4 (PBS) were obtained from Sigma-Aldrich ${ }^{\circledast}$. Oleic acid was from May \& Baker Ltd (England). Sodium phosphate monobasic monohydrated was acquired from Fluka (Germany). Sodium chloride was purchased from Panreac (Spain). Sodium acetate was from Fluka Analytical (Japan). Sodium hydroxide was obtained from VWR International (Belgium). Acetonitrile (Lichrosolv $®$ ) were obtained from MERK (Germany). DSPE-PEG.NH2 (1,2distearoyl-sn-glycero-3-phosphoethanolamine- $\mathrm{N}$-[amino(polyethylene glycol)-2000] (ammonium salt)) was purchased from (Avanti® Polar Lipids Inc.; Alabaster, Alabama, USA). SIF Powder was obtained from Biorelevant.com (Croydon, Surrey, UK). LDH Cytotoxicity Detection Kit was from Takara Bio Inc. (Shiga, Japan). Hanks' Balanced Salt Solution [-] CaCl2, [-] MgCl2 (HBSS), Dulbecco's Modified Eagle's Medium (DMEM) + GlutaMAXTM-I, 0.25\% Trypsin-EDTA (1X), Penicillin-Streptomycin (Pen Strep) and Heat Inactivated Fetal Bovine Serum (FBS) (origin: South America) were purchased from Gibco ${ }^{\circledR}$ by Life Technologies ${ }^{\top M}$ (UK).

All the weighting measurements performed using a Kern ACS-80-4 digital analytical balance (Kern \& Sohn; Balingen, Germany). pH measurement were obtained using a Crison pH meter GLP22 with a Crison 52-02 tip (Crison; Barcelona, Spain).

Ultrapure water was purified by an Ultra-pure water system (Milli-Q, Sartorius, Arium ${ }^{\circledR}$ pro, Sartorius Weighing Technology; Gettingen, Germany, Filters: Sartorius Arium ${ }^{\circledR}$ Cartige 1 and 2, Sartorius Stedin Biotech; Gettingen, Germany) by a reverse osmosis process.

\section{2 - Methods}

\subsubsection{Choice of solid lipid for improved drug loading}

Drug incorporation on a nanosystem is influenced by its capacity to dissolve on the system components [98, 103]. Thus, LNs drug solubility plays a major role, making the choice of lipids an importance step, in order to have a lipid or lipids mixture where the drug exhibit

high solubility. 
The analysis of oxaprozin qualitative solubility in the lipids was performed by melting an equal amount of different commercial lipids: Apifil, Cetyl Palmitate, Compritol HD5 A, Gelucire 33/01, Imwitor 308, Lipocire CM, Precirol Ato 5, Softisan 100, Superpolystrate, Witepsol E76, Witepsol E85, Witepsol H32, Witepsol S51 and Witepsol S58. The melting process was realized by slowly augmenting the temperature from $50^{\circ} \mathrm{C}$ to $70^{\circ} \mathrm{C}$ in order to assess the approximated phase transition temperature of the lipids. After the melting process, an amount of oxaprozin (always the same) was added to the melted lipids in order to qualitatively infer oxaprozin's solubility on the different lipids.

\subsubsection{Lipid nanoparticles synthesis}

Lipid nanoparticles were prepared by a modified free organic-solvent emulsification/sonication method combining high shear homogenization in an ultra-turrax, where a "pre-emulsion" with particles in the micrometer range are produced, followed by an ultra-sonication that will reduce the microparticles into nanoparticles resulting in a nanoemulsion. Cooling of the nanoemulsion allows lipid crystallization and the formation of the lipid nanosuspension [119].

Briefly, the lipid phase containing the desired lipid or lipids combination and the stabilizer Tween 60 was melted at a temperature above the phase transition temperature with or without stirring for better oxaprozin dissolution. Heating without stirring was performed in a water-bath (Medingen E5 Bath-Thermostat) and heating under stirring was performed in a top stirring hotplate (Barnstead International Thermolyne Cimarec $^{\circledR}$ 2; lowa, USA). For the synthesis of oxaprozin loaded lipid nanoparticles, oxaprozin was added to the lipid phase before the melting process. The molten lipids are dispersed in a specific amount of an aqueous phase at the same temperature (ultrapure water) by high-speed stirring in an ultra-turrax (Ystral X10/20 E3; Ballrechten-Dottingen, Germany) followed by sonication (Sonics and Material Vibra-Cell ${ }^{\mathrm{TM}}$ with a CV-18 probe; Newtown CT, USA). The solutions are cooled at room temperature and in some cases an extra amount of the aqueous phase at room temperature is added. The formulations are then stored at room temperature.

During the optimization process several lipids conjugations, aqueous phase quantities, stirring times during heat, high speed stirring and sonication parameters (time and potency) and post-synthesis aqueous phase addition quantities were tested in order to establish the best conditions for the lipid nanoparticles production.

The optimized formulation is synthesised by melting, under 10 minutes stirring, the lipid phase constituted by the solid lipid Precirol Ato 5 (170 mg), the liquid lipid Miglyol 182 (90 $\mathrm{mg}$ ) and the surfactant Tween $60(60 \mathrm{mg})$. For the production of oxaprozin loaded LNs, $30 \mathrm{mg}$ of oxaprozin are added to the lipid phase and this amount is deducted from the total amount of solid lipid. $4.4 \mathrm{~mL}$ of heated ultrapure water are added to the lipid phase and the emulsion mixed by ultra-turrax ( 30 seconds at 7000 RPM) followed by ultrasonication ( 5 minutes at $70 \%$ intensity). $4.4 \mathrm{~mL}$ of room temperature ultrapure water is then added and the emulsion cooled at room temperature. 


\subsubsection{Synthesis of disteroylphosphatidylethanolamine-poly(ethylene glycol)-folic acid (DSPE-PEG-FA) conjugate}

DSPE-PEG $2000-F A$ conjugate synthesis can be summarized in four main steps: activation of the folic acid, coupling to DSPE-PEG ${ }_{2000}-\mathrm{NH}_{2}$, purification and lyophilisation.

$\mathrm{N}$-Hydroxysuccinimide ester of folic acid (NHS-FA) was prepared by dissolving, under anhydrous conditions through overnight stirring in the dark, $1.0 \mathrm{~g}$ of folic acid (FA) into a mixture of $40 \mathrm{~mL}$ of anhydrous dimethyl sulfoxide (DMSO) and $0.5 \mathrm{~mL}$ of triethylamine (TEA). FA was then mixed with $0.5 \mathrm{~g}$ of dicyclohexylcarbodiimide (DCC) and $0.52 \mathrm{~g}$ of $\mathrm{N}$ hydroxysuccinimide (NHS) and stirred for further 18 hours in the dark. The resulting solution was then filtered with a $0.45 \mu \mathrm{M}$ filter in order to remove the precipitated side product (dicylcohexylurea (DCU)), DMSO and TEA were evaporated under vacuum [120, 121].

Coupling of the FA-NHS stock solution with DSPE-PEG ${ }_{2000}-\mathrm{NH}_{2}$ was accomplished by overnight stirring in the dark under anhydrous conditions of $2 \mathrm{~mL}$ of the resulting solution of activated folate with $50 \mathrm{mg}$ of DSPE-PEG $2000-\mathrm{NH}_{2}$ dissolved in $1 \mathrm{ml}$ of DMSO. After the process DMSO of removed by evaporation under vacuum and addition of $6 \mathrm{~mL}$ of water [121].

DSPE-PEG 2000 -FA conjugate was then dialyzed against $500 \mathrm{~mL}$ of ultrapure water using a dialysis membrane (Cellu.Sep ${ }^{\circledR}$ T1, 3500 NMWCO, Membrane Filtration Products, Inc.; Seguin, TX, USA) for 48 hours in order to remove the unconjugated FA [120].

The resulting solution was then lyophilized (Virtis Sentry 2.0 lyophilizator, SP Scientific) forming a yellow dry powder, the final DSPE-PEG ${ }_{2000}-\mathrm{FA}$ conjugate, stored at $4^{\circ} \mathrm{C}$.

\subsubsection{Synthesis of DSPE-PEG-FA functionalized LNs}

Functionalization of the synthesised LNs with DSPE-PEG-FA was obtained by adding the DSPE-PEG $2000-F A$ on the lipid phase in order for it to be encapsulated in the LNs matrix. The encapsulated DSPE-PEG ${ }_{2000}$-FA will be randomly placed among the nanoparticle so that despite a part of the encapsulated DSPE-PEG $2000-\mathrm{FA}$ may be placed on the interior of the particle a part of this DSPE-PEG $2000-F A$ will also be placed on the surface of the particle allowing an active macrophages targeting.

DSPE-PEG $2000-F A$ used to produce functionalized LNs was optimized and for the production of the final formulation, functionalized nanoparticles were obtained by adding $2 \%$ of the total lipid mass (lipids and surfactant) of DSPE-PEG 2000 -FA to the lipid phase.

\subsubsection{LNs characterization}

\subsubsection{Particle size measurements}

Particles in suspension present movement (Brownian movement), Dynamic Light Scattering (DLS), also known as photon correlation spectroscopy, use this movement in order to obtain the mean particles diameter and diameters distribution allowing also the identification of the different size populations present in solution.

Focusing a laser on the suspension, the movement of the particles will lead to variations on the light diffraction over time. These variations vary with the diffusion coefficient of the medium and with the particles size. Since bigger particles have slower movements than smaller particles they will diffract more the light. So analysing the movement of the particles by the light deviation and knowing the temperature and viscosity of the sample, DLS can calculate the hydrodynamic diameter of the particles on the solution [122]. 
The produced nanoparticles were characterized by its mean hydrodynamic diameter and size distribution (polydispersity index) in a Particle Size Analyzer (Brookhaven Instruments Corporation; Software: Particle Sizing v.5 Brookhaven Instruments; Holtsville, NY, USA) operating at a scattering angle of $90^{\circ}$, at $37^{\circ} \mathrm{C}$, with dust cut-off set to 30 and refractive index of the particles set to 1.33 . Measurements were performed by 6 runs of 2 minutes each and the mean size and polydispersity of the formulations were obtained. Before the measurements the formulations were diluted $(1: 800)$ and filtered with a single use syringe filter (Ministart, pore size 800 nm, Satorius Stedin Biotech; Goettingen, Germany).

\subsubsection{Zeta Potential measurements}

Zeta potential is a physical property exhibited by particles in suspension that determines its stability. Particles surface charge promotes interactions with the surrounding ions creating an unequal distribution of the solution ions in close proximity to the particle. This distribution leads to the formation of an inner zone were the solution ions are powerfully bonded to the surface and a more outer zone with a weakest connection. On this outer region the particle and the ions form a stable entity and the zeta potential is the potential associated at this theoretical limit [123].

Since particles with the same charge tend to repel themselves and since this potential is strictly connected to the particle surface charge, zeta potential is correlated with the suspension stability having particles with higher zeta potential, in modulus, higher tendency to repel themselves and consequently higher stability [124]. One can generally assume that particles have great stability for zeta potential values with absolute value superior to $30 \mathrm{mV}$ $[123,124]$.

Electrophoretic Light Scattering (ELS) is a method that calculates the zeta potential by light scattering. Two electrodes are inserted on the formulation creating an electric potential that attracts to the electrodes particles of the opposite charge. The movement created by this migration is analysed and the particles velocity measured by the light deviation, with this parameter is possible to calculate the particles zeta potential $[125,126]$.

Characterization of the particles zeta potential was performed in a Zeta Potential Analyser (ZetaPALS, Brookhaven Instruments Corporation, Software: PALS Zeta Potential Analyser v.5, Brookhaven Instruments; Holtsville, NY, USA) operating at a scattering angle of $90^{\circ}$, at $37^{\circ} \mathrm{C}$. Measurements were performed by 6 runs of 10 cycles each and the zeta potential and standard deviation of the formulations were obtained. Before the measurements the formulations were diluted (1:800) and filtered with a syringe filter $(800$ $\mathrm{nm})$.

\subsubsection{Oxaprozin Encapsulation Efficiency (EE)}

Oxaprozin encapsulation efficiency was determined by calculating the difference between the total amount of used oxaprozin to produce the formulations and the amount of free oxaprozin remaining on the aqueous phase.

Formulations where diluted in ultrapure water (1:50) and centrifuged in Amicom ${ }^{\circledR}$ Ultra Centrifugal Filter Devices, ultracel ${ }^{\circledR}$-50k (50000 NMWL) (MERK Milipore, Ltd; Cork, Ireland). Centrifugation (Heraeus Multifuge X1R centrifuge, Thermo Fisher Scientific; Germany) was performed with spin at $2664 \mathrm{~g}$ for 40 minutes at $20^{\circ} \mathrm{C}$. Free oxaprozin was present in the supernatant and was quantified by UV-Vis spectroscopy using a V-660 spectrophotometer 
(Jasco Corporation, Software: Spectra Manager v.2, Jasco Corporation; Easton, MD, USA) at $285 \mathrm{~nm}$.

Encapsulation efficiency was calculated as follows (Equation 1):

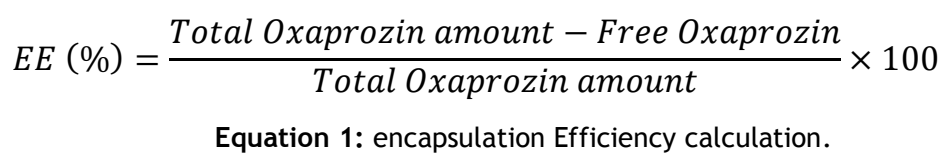

\subsubsection{Loading capacity (LC)}

LNs loading capacity was calculated using the oxaprozin encapsulation efficiency as follows (Equation 2):

$$
L C(\%)=\frac{E E \times \text { Total Oxaprozin amount }}{\text { Total lipid and surfactant amount }}
$$

\subsubsection{Transmission electron microscopy (TEM)}

In TEM thin samples are illuminated by an accelerated electrons beam and the electrons that are able to pass through the sample go into a system of electromagnetic lenses that enlarge and focus the image that is then recorded.

Samples were prepared by diluting the formulations in ultrapure water $(1: 50)$ and then 10 $\mu \mathrm{L}$ of this solution placed on a cooper-mesh grid and let to rest for 2 minutes at room temperature after which the water excess was removed. For contrasting, $10 \mu \mathrm{L}$ of $0.75 \%$ uranyl acetate solution was placed on the grid surface and let to rest for 30 seconds at room temperature. Solution excess was removed and the samples observed in a JEM-1400 Transmission Electron Microscope (JEOL Ltd., USA) with an accelerating voltage of $80 \mathrm{kV}$.

\subsubsection{In vitro release studies}

In vitro release studies were performed using a cellulose dialysis bag diffusion technique (Cellu.Sep ${ }^{\circledR}$ 11, 3500 NMWCO, Membrane Filtration Products, Inc.; Seguin, TX, USA) filled with $2 \mathrm{~mL}$ of the formulation (functionalized or non-functionalized oxaprozine loaded nanoparticles, 1:8 in ultrapure water).

In order to mimic the particles path in the body after oral administration, samples were incubated at $37^{\circ} \mathrm{C}$ under gentile stirring (IKA ${ }^{\circledR}$-Werke RT15-P Hot Stirring Plate; Germany). To simulate the transit from stomach to intestine samples were incubated first for 3 hours in 80 $\mathrm{mL}$ of fasted state simulated gastric fluid (FaSSGF: $\mathrm{NaCl} / \mathrm{HCl}$ solution, $\mathrm{pH} 1.2$ with SIF ${ }^{\oplus}$ Powder) and then placed for 4 hours in $80 \mathrm{~mL}$ of fasted state simulated intestinal fluid (FaSSIF: buffer solution containing potassium dihydrogen phosphate, $\mathrm{pH} 6.5$ with SIF ${ }^{\circledR}$ Powder). From this point some samples were placed in $80 \mathrm{ml}$ of simulated physiologic fluid (buffer solution containing monopotassium phosphate, $\mathrm{pH}$ 7.4) until the end of the experience in order to simulate the particles that never reach the inflamed regions. Other samples were placed until the end of the experience in simulated inflammatory fluid (buffer solution containing acetic acid and sodium acetate, $\mathrm{pH}$ 5.4) to simulate the release profile of the LNs in inflamed regions. Finally the rest of the samples were placed for 18 hours in the simulated physiologic fluid and then in the simulated inflammatory fluid until the end of the 
experience in order to mimic the release profile of LNs that circulate on the blood for a considerable period of time until reaching the inflamed regions.

At regular intervals, aliquots were collected for a UV-Vis microplate (UV flat bottom Microliter ${ }^{\circledR}$ plates, Thermo Electron Corporation; Vantaa, Finland) and replaced by an equal amount of fresh fluid (correspondent to the one that was being used). Oxaprozin release was analysed by UV-Vis spectroscopy using a microplate reader (BioTek Instruments Inc., Synergy HT, Software: Gen5 v1.08.4, BioTek Instruments Inc.; Winooski, USA) at 285 nm.

\subsubsection{Caco-2 cell culture}

Caco-2 cells (P.23 to P.32) were cultured at $37^{\circ} \mathrm{C}$ in an $5 \% \mathrm{CO}_{2}$ atmosphere (Unitherm $\mathrm{CO} 2$ Incubator 3503 Uniequip; Planegg, Germany) in Dulbecco's Modified Eagle Medium (DMEM) supplemented with 10\% Fetal Bovine Serum (FBS) and 1\% Penicillin-Streptomycin (Pen Strep). For every two to three days cells were washed with HBSS and the medium changed. Cells were subcultured at 80 to $90 \%$ confluence by chemical detachment with trypsin-EDTA and cells counted in a Neubauer chamber (Improved Neubauer Bright-line, Boeco; Germany) with $25 \%(\mathrm{v} / \mathrm{v})$ Trypan Blue solution $(0.4 \%(\mathrm{w} / \mathrm{v})$ in PBS) to exclude non-viable cells. Cells were then seeded at a density of 750000 cells per $75 \mathrm{~cm}^{2}$ flasks (Tissue culture flasks $250 \mathrm{~mL}$ (75 $\mathrm{cm}^{2}$ ), $0.2 \mu \mathrm{m}$ vented plug seal cap, Falcon ${ }^{\circledR}$, Becton Dickson; England) in $10 \mathrm{~mL}$ of DMEM.

\subsubsection{Cell viability assay}

The effect of the designed LNs on cell viability was measured using the methylthiazolyldiphenyl-tetratozium bromide (MTT) conversion assay. Caco-2 cells were seeded in 96-well tissue culture test microplates (Tissue Culture OrPlates, surface treated flat bottom, Orange Scientific; Belgium) at a density of 10000 cells/well in $100 \mu \mathrm{L}$ supplemented DMEM medium and incubated for 22 hours at $37^{\circ} \mathrm{C}$ in an $5 \% \mathrm{CO}_{2}$ atmosphere. $100 \mu \mathrm{L}$ of different concentrations $(5 \mu \mathrm{M}, 10 \mu \mathrm{M}, 50 \mu \mathrm{M}, 100 \mu \mathrm{M}, 500 \mu \mathrm{M}$ and $1000 \mu \mathrm{M})$ of free oxaprozin, oxaprozin loaded functionalized and non-functionalized LNs and the correspondent amounts of placebo functionalized and non-functionalized LNs, as well as the positive control (culture medium) and the negative control (Triton X-100 1\% ( $/ / v)$ in PBS) were then added and let to incubate with the cells for 4 hours. Supernatant was transferred for a 96-well tissue culture test microplate and stored for the realization of the cell toxicity study. $200 \mu \mathrm{L}$ of a MTT solution ( $5 \mathrm{mg} / \mathrm{mL}$ MTT in PBS stock solution diluted to a final concentration of 0.5 $\mathrm{mg} / \mathrm{mL}$ in culture medium) were then added to each cell seeded well and incubated for 3 hours at $37^{\circ} \mathrm{C}$ in an $5 \% \mathrm{CO}_{2}$ atmosphere to allow the formation of formazan crystals. MTT was then rejected by plate inversion and $200 \mu \mathrm{L}$ of DMSO were added and incubated for 15 minutes at $37^{\circ} \mathrm{C}$ in a $5 \% \mathrm{CO}_{2}$ atmosphere protected from light in order to elute blue formazan from cells and solubilise the formazan crystals. Absorbance was read using a microplate reader at $550 \mathrm{~nm}$ and $690 \mathrm{~nm}$ for background subtraction. Cell viability was determined according as follows (Equation 3):

$$
\begin{gathered}
\text { Cell viability }(\%)=\frac{\text { Experimental value }}{\text { Mean positive control value }} \times 100 \\
\text { Equation } 3 \text { : Cell viability calculation. }
\end{gathered}
$$




\subsubsection{Cell toxicity assay}

The cell toxicity assay realized analyzes the presence of Lactase Dyhydrogenase (LDH) on the culture medium. LDH is a cytoplasmatic enzyme present in most cells and is released to the culture medium in cells with cytoplasmatic membrane damages. Using a LDH Cytotoxicity Detection Kit, which will react with the LDH forming formazan, one can quantify the formazan which can be directly correlated with the LDH present on the culture medium assessing the cell death.

The first steps of the LDH assay are equal to those performed on the cell viability assay until the end of the incubation period of the samples with the cells. From here on the LDH assay uses the recovered supernatant that resulted from the incubation. The recovered microplates were centrifuged (Sigma 3k-2 microplates centrifuge) in order to deposit the cellular remains for 10 minutes at $250 \mathrm{~g}$, at room temperature. After centrifugation, $100 \mu \mathrm{L}$ of the supernatant were carefully transferred for another 96-well tissue culture test microplate and $100 \mu \mathrm{L}$ of the LDH Cytotoxicity Detection Kit reaction mixture added to the wells and let to incubate in the dark for 15 minutes at room temperature. Absorbance was then read using a microplate reader at $490 \mathrm{~nm}$ and 690 for background subtraction and cytotoxicity calculated having into consideration that for this assay the positive control were the cells treated with $1 \%$ Triton $\mathrm{X}-100(\mathrm{v} / \mathrm{v})$ in PBS and the negative control were the cells treated with culture medium. Cell cytotoxicity was determined according as follows (Equation 4):

$$
\begin{gathered}
\text { Cell cytotoxicity }(\%)=\frac{\text { Experimental value }}{\text { Mean positive control value }} \times 100 \\
\text { Equation } 4 \text { : Cell cytotoxicity calculation. }
\end{gathered}
$$

\subsubsection{Cell permeability studies}

Caco-2 cells were seeded on Transwell diffusion cells with a density of 100000 cells per $\mathrm{cm}^{2}$ (45000 cells per insert of $4.67 \mathrm{~cm}^{2}$ polycarbonate membrane with $3.0 \mu \mathrm{m}$ porosity) in supplemented DMEM culture medium and maintained in culture medium for 21 days. After 21 days of culture, Caco-2 cells form an absorptive polarized monolayer developing an apical brush border and enzyme segregation, so that they can be used to mimic the intestinal endothelium [127]. For every three to four days cells were observed on an trinocular inverted microscope (Motic AE2000 TRI coupled with a camera Moticam 5 MP; Spain) and the transepithelial electrical resistance (TEER) measured on the polycarbonate filters of the Transwell diffusion cells using an epithelial voltammeter (Epithelial voltohmmeter EVOM ${ }^{2}$, World Precision Instruments, Inc.; USA) to evaluate thigh junctions formation. Cells were also washed twice with PBS and the culture medium substituted. At the $21^{\text {st }}$ day TEER must be over $200 \Omega . \mathrm{cm}^{2}$, the reference value which indicates that the monolayer presents characteristics similar to those of the intestinal mucosa [128].

For the permeability studies the culture medium was removed and the cells, the insert and receptor well washed twice with PBS. Fasted state simulated intestinal fluid (FaSSIF: buffer solution containing potassium dihydrogen phosphate, $\mathrm{pH} 6.5$ with SIF ${ }^{\circledR}$ Powder) and the reference medium PBS were added to the correspondent inserts and receptor wells. Free oxaprozin and functionalized and non-functionalized oxaprozin loaded LNs at $100 \mu \mathrm{M}$ concentration (determined by the cell viability and cytotoxicity assays) diluted on the correspondent medium were incubated on the inserts for 4 hours at $37^{\circ} \mathrm{C}$ in a $5 \% \mathrm{CO}_{2}$ atmosphere. At different times, $160 \mu \mathrm{L}$ aliquots were collected for a 96-well UV-Vis 
microplate from the receptor well and substituted by the same amount of the correspondent medium, along with aliquots from the insert at the initial and final time. TEER measurements were also performed at the same times in order to evaluate cell viability and the opening of the tight junctions during the 4 hours assay. At the end of the study, $40 \mu \mathrm{L}$ of a mixture of acetonitrile $(92 \%)$ and acetic acid (8\%) were added at the aliquots in order to dissolve the lipid matrix of the LNs and expose oxaprozin in the solution to be quantified. Oxaprozin quantification was performed by UV-Vis spectroscopy using a microplate reader, at wavelength of $285 \mathrm{~nm}$.

Apparent permeability coefficient $\left(P_{\text {app }}\right)$ was calculated as follows (Equation 5):

$$
P_{a p p}(\mathrm{~cm} / \mathrm{s})=\frac{Q}{A \times C_{0} \times t}
$$

Equation 5: Apparent permeability coefficient calculation.

Where $Q$ is the total amount of permeated oxaprozin $(\mu \mathrm{g}), \mathrm{A}$ is the diffusion area $\left(\mathrm{cm}^{2}\right)$, $C_{0}$ is the initial oxaprozin concentration $(\mu \mathrm{g} / \mathrm{mL})$ and $t$ is the time of the experience $(\mathrm{s})$.

\subsubsection{Statistical analysis}

Statistical analyses were performed using IBM ${ }^{\circledR}$ SPSS ${ }^{\circledR}$ Statistics software (v.22.0.0.0; IBM, Armonk, NY, USA). The measurements were repeated at least three times and data expressed as mean \pm SD. Data were analyzed using one-way analysis of variance (ANOVA) and differences between groups compared by Bonferroni and Tukey post-hoc tests with a $P$ value of $<0.05$ considered statistically significant. 


\section{Chapter 3}

\section{Results and Discussion}

\section{1 - Choice of solid lipid for improved drug loading}

Drug solubility in the lipid phase of the lipid nanoparticles plays an important role on the entrapment efficiency, drug loading and on the release profile of the lipid nanoparticles. Phase transition temperature is also an important feature since it correlated with the stability of the crystalline structure of the lipid nanoparticle. Table 2 presents the lipids that were tested, their phase transition temperature and also their relative ability to solubilise oxaprozin.

Table 2: Lipids phase transition temperature and qualitative oxaprozin dissolution capacity.

\begin{tabular}{|c|c|c|c|c|}
\hline \multirow{3}{*}{ Lipid } & \multicolumn{3}{|c|}{ Liquid at } & \multirow{3}{*}{$\begin{array}{c}\text { Oxaprozin } \\
\text { dissolution capacity }\end{array}$} \\
\hline & $50^{\circ}$ & $60^{\circ}$ & $70^{\circ}$ & \\
\hline & $\mathrm{C}$ & $C$ & $C$ & \\
\hline Apifil & $x$ & $\checkmark$ & $\checkmark$ & - \\
\hline Cetyl Palmitate & $x$ & $x$ & $\checkmark$ & - \\
\hline Compritol HD5 A & $x$ & $x$ & $\checkmark$ & + \\
\hline Gelucire 33/01 & $\checkmark$ & $\checkmark$ & $\checkmark$ & - \\
\hline Imwitor 308 & $\checkmark$ & $\checkmark$ & $\checkmark$ & +++ \\
\hline Lipocire CM & $\checkmark$ & $\checkmark$ & $\checkmark$ & ++ \\
\hline Precirol Ato 5 & $x$ & $x$ & $\checkmark$ & + \\
\hline Softisan 100 & $\checkmark$ & $\checkmark$ & $\checkmark$ & - \\
\hline Superpolystrate & $\checkmark$ & $\checkmark$ & $\checkmark$ & +++ \\
\hline Witepsol E76 & $x$ & $x$ & $\checkmark$ & - \\
\hline Witepsol E85 & $\checkmark$ & $\checkmark$ & $\checkmark$ & + \\
\hline Witepsol H32 & $\checkmark$ & $\checkmark$ & $\checkmark$ & - \\
\hline Witepsol S51 & $x$ & $x$ & $\checkmark$ & - \\
\hline Witepsol S58 & $\checkmark$ & $\checkmark$ & $\checkmark$ & ++ \\
\hline
\end{tabular}

Imwitor 308 and Superpolystrate with phase transition temperature inferior to $50^{\circ} \mathrm{C}$ were the only lipids with a very good apparent oxaprozin dissolution capacity followed by Lipocire $\mathrm{CM}$ and Witepsol S58 with phase transition temperature around $50^{\circ} \mathrm{C}$ with good oxaprozin solubilisation and Compritol HD5 A, Precirol Ato 5 with phase transition temperature around $70^{\circ} \mathrm{C}$ and Witepsol E85 with phase transition temperature close to $50^{\circ} \mathrm{C}$ shown low oxaprozin solubility. The other lipids were not able to solubilise oxaprozin. 


\section{2 - Lipid nanoparticles synthesis and optimization}

Synthesis process was optimized in order to obtain a workable liquid nanoparticle suspension with particles with a diameter larger than $200 \mathrm{~nm}$ to be taken by the macrophages and a zeta potential over $|30 \mathrm{mV}|$ so that the particles could be stable, i.e., so that they do not tend to form aggregates [123, 124]. Encapsulation efficiency and loading capacity were also analysed since they represent the amount of drug entrapped on the lipid nanoparticles. In the end of the process, the functionalization of the particles with folic acid was also optimized.

Table 3 summarizes the optimization process in which several combinations of lipids, ultra-turrax velocities and time, sonication amplitudes and time and also water contents were tested and analysed.

Briefly, the solid lipids that demonstrated an apparent capacity to dissolve oxaprozin were used to synthesize SLNs and NLCs (LN1 to LN7), however they were not able to dissolve the amount of oxaprozin used in the synthesis.

The lipids Imwitor 308 and Superpolystrate shown a good capacity to dissolve oxaprozin, however, since their phase transition temperature is beneath $50^{\circ} \mathrm{C}$ they were used simultaneously with Cetyl Palmitate, which as a phase transition temperature around $70^{\circ} \mathrm{C}$ in order to promote a higher stability of the particles at temperatures above the room temperature. Several combinations and synthesis conditions (LN9 to LN23) were tested without success to obtain a workable liquid suspension, i.e. with low viscosity, and nanoparticles with the desired diameter and zeta-potential. However, we verified that under stirring, Cetyl Palmitate was able to dissolve oxaprozin, so the synthesis of SLNs and NLCs with this lipid was optimized (LN24 to LN 29) resulting in the SLN synthesis process (LN41) that shown to be able to produce nanoparticles with the desired characteristics.

The synthesis of SLNs combining Imwitor 308 and Superpolystrate with Precirol Ato 5, shown good capacity to dissolve oxaprozin (LN30 and LN31), nevertheless it was not possible to obtain a workable liquid suspension and thereby the two formulations were discarded. However, since Precirol Ato 5 was able to dissolve oxaprozin, the synthesis of SLNs and NLCs were optimized (LN33 to LN40) resulting in the NLC synthesis process described in LN43 that shown to be able to produce nanoparticles with the desired characteristics.

Two type of workable liquid nanoparticles suspensions with particles with diameter superior to $200 \mathrm{~nm}$ and zeta-potential over |30mV| (LN41 e LN43) were produced. Both types of particles possessed encapsulation efficiency superior to $95 \%$ and loading capacity around $6 \%$. We then tried, with success, to augment the amount of oxaprozin in the particles (LN45 and LN46) maintaining the good encapsulation efficiency and increasing the loading capacity to around $9 \%$.

The next step was the production of functionalized nanoparticles from the formulations LN41 and LN43, with different amounts of DSPE-PEG ${ }_{2000}-$ FA (LN47 to LN50). Cetyl Palmitate functionalized particles tend to form a deposit of DSPE-PEG 2000 -FA even with small amounts of the compound. Besides NLCs generally have better propertied than SLNs as described above so, as a consequence the optimization process was continued with the NLCs formulations containing Precirol Ato 5 (LN51 to LN55), resulting on the production method summarized in LN55 that allowed the synthesis of functionalized NLCs with the desired characteristics and high encapsulation efficiency and loading capacity. 
Table 3: List of the synthesised LNs during the optimization process.

\begin{tabular}{|c|c|c|c|c|c|c|c|c|c|c|c|c|c|c|c|}
\hline \multirow[b]{2}{*}{$\begin{array}{l}\mathrm{LN} \\
\text { code }\end{array}$} & \multirow[b]{2}{*}{$\begin{array}{l}\mathrm{LN} \\
\text { type }\end{array}$} & \multirow[b]{2}{*}{ Constitution } & \multirow[b]{2}{*}{$\begin{array}{l}\text { Mass } \\
(\mathrm{mg})\end{array}$} & \multirow[b]{2}{*}{$\begin{array}{l}\text { Strirring } \\
\text { (min.) }\end{array}$} & \multirow[b]{2}{*}{$\begin{array}{l}\text { Water } \\
(\mathrm{mL})\end{array}$} & \multicolumn{2}{|c|}{ Ultra-Turrax } & \multicolumn{2}{|c|}{ Sonication } & \multirow[b]{2}{*}{$\begin{array}{l}\text { Post-synthesis } \\
\text { water addition } \\
(\mathrm{mL})\end{array}$} & \multirow[b]{2}{*}{$\begin{array}{l}\text { Diameter } \\
(\mathrm{nm})\end{array}$} & \multirow[b]{2}{*}{ Polydispersity } & \multirow[b]{2}{*}{$\begin{array}{l}\text { Zeta-Potential } \\
(\mathrm{mV})\end{array}$} & \multirow[b]{2}{*}{$\begin{array}{l}\text { Encapsulation } \\
\text { efficiency (\%) }\end{array}$} & \multirow[b]{2}{*}{$\begin{array}{l}\text { Loading } \\
\text { capacity (\%) }\end{array}$} \\
\hline & & & & & & $\begin{array}{l}\text { Velocity } \\
\text { (RPM) }\end{array}$ & $\begin{array}{c}\text { Time } \\
\text { (s) }\end{array}$ & $\begin{array}{c}\text { Amplitude } \\
(\%)\end{array}$ & $\begin{array}{l}\text { Time } \\
\text { (min.) }\end{array}$ & & & & & & \\
\hline \multirow{3}{*}{ LN1 } & \multirow{3}{*}{ SLN } & Witepsol S58 & 500 & & & & & & & & & & & & \\
\hline & & Tween 60 & 100 & -- & -- & -- & -- & -- & -- & -- & -- & -- & -- & -- & -- \\
\hline & & Oxaprozin & 20 & & & & & & & & & & & & \\
\hline \multirow{4}{*}{ LN2 } & \multirow{4}{*}{ NLC } & Witepsol S58 & 350 & \multirow{4}{*}{--} & \multirow{4}{*}{--} & \multirow{4}{*}{$-\cdot$} & \multirow{4}{*}{--} & \multirow{4}{*}{--} & \multirow{4}{*}{--} & \multirow{4}{*}{--} & \multirow{4}{*}{-- } & \multirow{4}{*}{--} & \multirow{4}{*}{--} & \multirow{4}{*}{--} & \multirow{4}{*}{--} \\
\hline & & Miglyol 182 & 150 & & & & & & & & & & & & \\
\hline & & Tween 60 & 100 & & & & & & & & & & & & \\
\hline & & Oxaprozin & 20 & & & & & & & & & & & & \\
\hline & & Witepsol E85 & 500 & & & & & & & & & & & & \\
\hline LN3 & SLN & Tween 60 & 100 & -- & -- & -- & -- & -- & -- & -- & -- & -- & -- & -- & -- \\
\hline & & Oxaprozin & 20 & & & & & & & & & & & & \\
\hline & & Witepsol E85 & 350 & & & & & & & & & & & & \\
\hline NA & $N=C$ & Miglyol 182 & 150 & & & & & & & & & & & & \\
\hline LN4 & INLC & Tween 60 & 100 & -- & - & - & - & - & - & - & - & -- & - & - & - \\
\hline & & Oxaprozin & 20 & & & & & & & & & & & & \\
\hline & & Lipocire CM & 500 & & & & & & & & & & & & \\
\hline LN5 & SLN & Tween 60 & 100 & -- & -- & -- & -- & -- & -- & -- & -- & -- & -- & -- & -- \\
\hline & & Oxaprozin & 20 & & & & & & & & & & & & \\
\hline & & Lipocire CM & 350 & & & & & & & & & & & & \\
\hline ING & NIC & Miglyol 182 & 150 & -. & $\ldots$ & .. & $\ldots$ & -. & $\ldots$ & $\ldots$ &. & $\ldots$ & $\ldots$ & $\ldots$ & $\ldots$ \\
\hline Livo & TVLC & Tween 60 & 100 & & & & & & & & & & & & \\
\hline & & Oxaprozin & 20 & & & & & & & & & & & & \\
\hline
\end{tabular}




\begin{tabular}{|c|c|c|c|c|c|c|c|c|c|c|c|c|c|c|c|}
\hline \multirow{3}{*}{ LN7 } & \multirow{3}{*}{ SLN } & Witepsol H32 & 500 & \multirow{3}{*}{--} & \multirow{3}{*}{--} & \multirow{3}{*}{--} & \multirow{3}{*}{--} & \multirow{3}{*}{--} & \multirow{3}{*}{-- } & \multirow{3}{*}{--} & \multirow{3}{*}{--} & \multirow{3}{*}{--} & \multirow{3}{*}{--} & \multirow{3}{*}{--} & \multirow{3}{*}{-- } \\
\hline & & Tween 60 & 100 & & & & & & & & & & & & \\
\hline & & Oxaprozin & 20 & & & & & & & & & & & & \\
\hline \multirow{3}{*}{ LN8 } & \multirow{3}{*}{ SLN } & Cetyl Palmitate & 500 & \multirow{3}{*}{--} & \multirow{3}{*}{--} & \multirow{3}{*}{--} & \multirow{3}{*}{$-\cdot$} & \multirow{3}{*}{--} & \multirow{3}{*}{--} & \multirow{3}{*}{--} & \multirow{3}{*}{--} & \multirow{3}{*}{\multicolumn{2}{|c|}{--}} & \multirow{3}{*}{--} & \multirow{3}{*}{--} \\
\hline & & Tween 60 & 100 & & & & & & & & & & & & \\
\hline & & Oxaprozin & 20 & & & & & & & & & & & & \\
\hline \multirow{4}{*}{ LN9 } & & Cetyl Palmitate & 250 & & & & & & & & & & & & \\
\hline & SLN & Imwitor 308 & 250 & & 4.4 & 12000 & 30 & 80 & 5 & .. & $1354+31$ & $0.28+0.01$ & $-24+1$ & 93.7 & 3.1 \\
\hline & & Tween 60 & 100 & & 1.7 & 12000 & $\pi 0$ & & & & זער & 0.2010 .01 & $21-1$ & . & \\
\hline & & Oxaprozin & 20 & -- & & & & & & & & & & & \\
\hline & & Cetyl Palmitate & 250 & & & & & & & & & & & & \\
\hline LN10 & SLN & Superpolystrate & 250 & -- & 4.4 & 12000 & 30 & 80 & 5 & -- & $337 \pm 4$ & $0.269 \pm 0.005$ & $-38.7 \pm 0.8$ & -- & -- \\
\hline & & Tween 60 & 100 & & & & & & & & & & & & \\
\hline & & Oxaprozin & 20 & & & & & & & & & & & & \\
\hline & & Cetyl Palmitate & 250 & & & & & & & & & & & & \\
\hline LN11 & SLN & Imwitor 308 & 250 & -.- & 4.4 & 12000 & 120 & 70 & 15 & .- & $1932 \pm 280$ & $0.07 \pm 0.03$ & $-25 \pm 1$ & 91.6 & 3.1 \\
\hline & & Tween 60 & 100 & & & & & & & & & & & & \\
\hline & & Oxaprozin & 20 & & & & & & & & & & & & \\
\hline & & Cetyl Palmitate & 250 & & & & & & & & & & & & \\
\hline IN12 & SIN & Superpolystrate & 250 & .. & 44 & 12000 & 120 & 70 & 15 & .. & $1000+45$ & $0363+0.006$ & $-46+1$ & .- & $\ldots$ \\
\hline & & Tween 60 & 100 & & T. & & & & & & & 0.0050 .000 & - & & \\
\hline & & Oxaprozin & 20 & & & & & & & & & & & & \\
\hline & & Cetyl Palmitate & 250 & & & & & & & & & & & & \\
\hline IN13 & SIN & Superpolystrate & 250 & $\ldots$ & 88 & 12000 & 120 & 70 & 15 & .. & $366+8$ & $0.32+0.01$ & $-36.4+0.8$ & 94.3 & 31 \\
\hline Livis & JLi & Tween 60 & 100 & & 0.0 & 12000 & 120 & 10 & J & - & $200 \pm 0$ & $0.32 \pm 0.01$ & $-36.4 \pm 0.8$ & 94.3 & 3.1 \\
\hline & & Oxaprozin & 20 & & & & & & & & & & & & \\
\hline
\end{tabular}




\begin{tabular}{|c|c|c|c|c|c|c|c|c|c|c|c|c|c|c|c|}
\hline \multirow{4}{*}{ LN14 } & \multirow{4}{*}{ SLN } & Cetyl Palmitate & 400 & \multirow{4}{*}{--} & \multirow{4}{*}{4.4} & \multirow{4}{*}{12000} & \multirow{4}{*}{30} & \multirow{4}{*}{80} & \multirow{4}{*}{5} & \multirow{4}{*}{--} & \multirow{4}{*}{$124 \pm 5$} & \multirow{4}{*}{$0.32 \pm 0.02$} & \multirow{4}{*}{$-10 \pm 3$} & \multirow{4}{*}{99.4} & \multirow{4}{*}{3.3} \\
\hline & & Superpolystrate & 100 & & & & & & & & & & & & \\
\hline & & Tween 60 & 100 & & & & & & & & & & & & \\
\hline & & Oxaprozin & 20 & & & & & & & & & & & & \\
\hline \multirow{4}{*}{ LN15 } & \multirow{4}{*}{ SLN } & Cetyl Palmitate & 400 & \multirow{4}{*}{-- } & \multirow{4}{*}{4.4} & \multirow{4}{*}{12000} & \multirow{4}{*}{30} & \multirow{4}{*}{80} & \multirow{4}{*}{3} & \multirow{4}{*}{--} & \multirow{4}{*}{$679 \pm 122$} & \multirow{4}{*}{$0.17 \pm 0.08$} & \multirow{4}{*}{$-16 \pm 3$} & \multirow{4}{*}{99.5} & \multirow{4}{*}{3.} \\
\hline & & Superpolystrate & 100 & & & & & & & & & & & & \\
\hline & & Tween 60 & 100 & & & & & & & & & & & & \\
\hline & & Oxaprozin & 20 & & & & & & & & & & & & \\
\hline \multirow{4}{*}{ LN16 } & & Cetyl Palmitate & 400 & & & & & & & & & & & & \\
\hline & SIN & Superpolystrate & 100 & $\pi$ & 6 & 12000 & 30 & 80 & 5 & 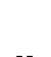 & $230+7$ & 030 & $23+1$ & 990 & $332+3$ \\
\hline & & Tween 60 & 100 & & 0 & 12000 & 30 & ov & $J$ & & $2 \angle 0 \pm 1$ & $0.29 \pm 0.03$ & $-\angle 3 \pm 1$ & 99.0 & 3.3 \\
\hline & & Oxaprozin & 20 & & & & & & & & & & & & \\
\hline & & Cetyl Palmitate & 400 & & & & & & & & & & & & \\
\hline 1017 & $\mathrm{cLS}_{\mathrm{s}}$ & Superpolystrate & 100 & & 6 & 12000 & 0 & 80 & 2 & & 221 & ב0 020 & $240+0$ & 000 & 23 \\
\hline LIVII & $\mathrm{JLN}$ & Tween 60 & 100 & $\cdot-$ & 6 & 12000 & 30 & 80 & 3 & -- & $\angle 34 \pm 6$ & $0.28 \pm 0.03$ & $-24.0 \pm 0.5$ & 99.0 & 3.3 \\
\hline & & Oxaprozin & 20 & & & & & & & & & & & & \\
\hline & & Cetyl Palmitate & 230 & & & & & & & & & & & & \\
\hline $1 \times 40$ & $\mathrm{css}$ & Superpolystrate & 250 & 20 & 0 & 12000 & 20 & 80 & & & & & & & \\
\hline LN18 & SLN & Tween 60 & 100 & 20 & 8 & 12000 & 30 & 80 & 5 & -- & -- & -- & -- & -- & -- \\
\hline & & Oxaprozin & 20 & & & & & & & & & & & & \\
\hline & & Cetyl Palmitate & 230 & & & & & & & & & & & & \\
\hline 1910 & chs & Superpolystrate & 250 & 30 & 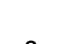 & (1) & 20 & & 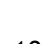 & & & & & & \\
\hline LN19 & SLN & Tween 60 & 100 & 20 & 8 & 12000 & 120 & 10 & 10 & $\cdots$ & -- & -- & -- & -- & -- \\
\hline & & Oxaprozin & 20 & & & & & & & & & & & & \\
\hline
\end{tabular}




\begin{tabular}{|c|c|c|c|c|c|c|c|c|c|c|c|c|c|c|c|}
\hline \multirow{4}{*}{ LN20 } & \multirow{4}{*}{ SLN } & Cetyl Palmitate & 230 & \multirow{4}{*}{20} & \multirow{4}{*}{8} & \multirow{4}{*}{12000} & \multirow{4}{*}{120} & \multirow{4}{*}{70} & \multirow{4}{*}{15} & \multirow{4}{*}{--} & \multirow{4}{*}{--} & \multirow{4}{*}{--} & \multirow{4}{*}{--} & \multirow{4}{*}{--} & \multirow{4}{*}{--} \\
\hline & & Superpolystrate & 250 & & & & & & & & & & & & \\
\hline & & Tween 60 & 100 & & & & & & & & & & & & \\
\hline & & Oxaprozin & 20 & & & & & & & & & & & & \\
\hline \multirow{4}{*}{ LN21 } & \multirow{4}{*}{ SLN } & Cetyl Palmitate & 230 & \multirow{4}{*}{20} & \multirow{4}{*}{8.8} & \multirow{4}{*}{12000} & \multirow{4}{*}{120} & \multirow{4}{*}{70} & \multirow{4}{*}{10} & \multirow{4}{*}{--} & \multirow{4}{*}{--} & \multirow{4}{*}{--} & \multirow{4}{*}{--} & \multirow{4}{*}{--} & \multirow{4}{*}{-- } \\
\hline & & Superpolystrate & 200 & & & & & & & & & & & & \\
\hline & & Tween 60 & 100 & & & & & & & & & & & & \\
\hline & & Oxaprozin & 20 & & & & & & & & & & & & \\
\hline & & Cetyl Palmitate & 230 & & & & & & & & & & & & \\
\hline $1 N 22$ & SIN & Superpolystrate & 200 & 20 & 88 & 12000 & 120 & 70 & 15 & & & & & & \\
\hline & JLIV & Tween 60 & 100 & 20 & 0.0 & 12000 & $1 \angle 0$ & 10 & 15 & -- & $\cdots$ & -- & -- & -- & - \\
\hline & & Oxaprozin & 20 & & & & & & & & & & & & \\
\hline & & Cetyl Palmitate & 180 & & & & & & & & & & & & \\
\hline LN23 & SLN & Superpolystrate & 90 & 20 & 8.8 & 7000 & 30 & 70 & 5 & .- & $848+22$ & $0.375+0.006$ & $-42+2$ & 98.5 & 6.0 \\
\hline & & Tween 60 & 60 & & & & & & & & & & & & \\
\hline & & Oxaprozin & 20 & & & & & & & & & & & & \\
\hline & & Cetyl Palmitate & 270 & & & & & & & & & & & & \\
\hline LN24 & SLN & Tween 60 & 60 & 20 & 8.8 & 7000 & 30 & 70 & 5 & -- & $283 \pm 5$ & $0.15 \pm 0.02$ & $-33.8 \pm 0.8$ & 98.3 & 6.0 \\
\hline & & Oxaprozin & 20 & & & & & & & & & & & & \\
\hline & & Cetyl Palmitate & 180 & & & & & & & & & & & & \\
\hline $1 N 25$ & $\mathrm{~N} / \mathrm{C}$ & Miglyol 182 & 90 & 20 & 00 & 7000 & 30 & 70 & 5 & & 25 & $016+0 ?$ & 40 & 006 & 200 \\
\hline LN25 & $\mathrm{NLC}$ & Tween 60 & 60 & 20 & 8.8 & 1000 & 30 & 10 & 5 & $\cdot-$ & $25 \pm 2$ & $0.16 \pm 0.02$ & $-40.2 \pm 0.4$ & 98.6 & 29.8 \\
\hline & & Oxaprozin & 20 & & & & & & & & & & & & \\
\hline & & Cetyl Palmitate & 180 & & & & & & & & & & & & \\
\hline $1 N 26$ & 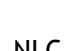 & Oleic Acid & 90 & 20 & 88 & 7000 & 30 & 70 & 5 & & $145+1$ & $011+01$ & $352+04$ & 082 & 60 \\
\hline LIN $\angle 0$ & NLLC & Tween 60 & 60 & 20 & 0.0 & 7000 & 30 & 10 & $J$ & -- & $145 \pm 1$ & $0.11 \pm 0.01$ & $-30.2 \pm 0.4$ & 90.3 & 0.0 \\
\hline & & Oxaprozin & 20 & & & & & & & & & & & & \\
\hline
\end{tabular}




\begin{tabular}{|c|c|c|c|c|c|c|c|c|c|c|c|c|c|c|c|}
\hline \multirow{3}{*}{ LN27 } & \multirow{3}{*}{ SLN } & Cetyl Palmitate & 270 & \multirow{3}{*}{10} & \multirow{3}{*}{8.8} & \multirow{3}{*}{7000} & \multirow{3}{*}{30} & \multirow{3}{*}{70} & \multirow{3}{*}{8} & \multirow{3}{*}{--} & \multirow{3}{*}{$278 \pm 4$} & \multirow{3}{*}{$0.15 \pm 0.02$} & \multirow{3}{*}{$-43 \pm 1$} & \multirow{3}{*}{--} & \multirow{3}{*}{--} \\
\hline & & Tween 60 & 60 & & & & & & & & & & & & \\
\hline & & Oxaprozin & 20 & & & & & & & & & & & & \\
\hline \multirow{3}{*}{ LN28 } & \multirow{3}{*}{ SLN } & Cetyl Palmitate & 270 & \multirow{3}{*}{10} & \multirow{3}{*}{8.8} & \multirow{3}{*}{7000} & \multirow{3}{*}{30} & \multirow{3}{*}{70} & \multirow{3}{*}{7} & \multirow{3}{*}{--} & \multirow{3}{*}{$284 \pm 3$} & \multirow{3}{*}{$0.190 \pm 0.005$} & \multirow{3}{*}{$-42.7 \pm 0.8$} & \multirow{3}{*}{--} & \multirow{3}{*}{--} \\
\hline & & Tween 60 & 60 & & & & & & & & & & & & \\
\hline & & Oxaprozin & 20 & & & & & & & & & & & & \\
\hline & & Cetyl Palmitate & 270 & & & & & & & & & & & & \\
\hline LN29 & SLN & Tween 60 & 60 & 10 & 8.8 & 7000 & 30 & 70 & 6 & -- & $283 \pm 6$ & $0.16 \pm 0.01$ & $-41.7 \pm 0.5$ & -- & -- \\
\hline & & Oxaprozin & 20 & & & & & & & & & & & & \\
\hline & & Precirol Ato 5 & 400 & & & & & & & & & & & & \\
\hline LN30 & SLN & Imwitor 308 & 100 & 30 & 4.4 & 12000 & 30 & 80 & 5 & -- & -- & -- & -- & -- & -- \\
\hline & & Tween 60 & 100 & & & & & & & & & & & & \\
\hline & & Precirol Ato 5 & 400 & & & & & & & & & & & & \\
\hline LN31 & SLN & Superpolystrate & 100 & 30 & 4.4 & 12000 & 30 & 80 & 5 & -- & -- & -- & -- & -- & -- \\
\hline & & Tween 60 & 100 & & & & & & & & & & & & \\
\hline $1 N 32$ & SIN & Precirol Ato 5 & 500 & 30 & 4 & 12000 & 30 & 80 & 5 & & & & & & \\
\hline LINJL & JLIN & Tween 60 & 100 & 30 & 4.4 & 12000 & 30 & 80 & 5 & $\cdots$ & -- & -- & -- & $-\cdot$ & -- \\
\hline & & Precirol Ato 5 & 500 & & & & & & & & & & & & \\
\hline LN33 & SLN & Tween 60 & 100 & 30 & 4.4 & 12000 & 30 & 80 & 5 & -- & $1420 \pm 164$ & $0.24 \pm 0.07$ & $-21 \pm 1$ & -- & -- \\
\hline & & Oxaprozin & 20 & & & & & & & & & & & & \\
\hline & & Precirol Ato 5 & 270 & & & & & & & & & & & & \\
\hline LN34 & SLN & Tween 60 & 60 & 10 & 8.8 & 7000 & 30 & 70 & 5 & -- & -- & -- & -- & -- & -- \\
\hline & & Oxaprozin & 20 & & & & & & & & & & & & \\
\hline & & Precirol Ato 5 & 350 & & & & & & & & & & & & \\
\hline LN35 & NLC & Oleic Acid & 150 & 30 & 4.4 & 12000 & 120 & 70 & 15 & -- & -- & -- & -- & -- & -- \\
\hline & & Tween 60 & 100 & & & & & & & & & & & & \\
\hline
\end{tabular}




\begin{tabular}{|c|c|c|c|c|c|c|c|c|c|c|c|c|c|c|c|}
\hline \multirow{3}{*}{ LN36 } & \multirow{3}{*}{ NLC } & Precirol Ato 5 & 350 & \multirow{3}{*}{30} & \multirow{3}{*}{4.4} & \multirow{3}{*}{12000} & \multirow{3}{*}{120} & \multirow{3}{*}{70} & \multirow{3}{*}{15} & \multirow{3}{*}{--} & \multirow{3}{*}{$802 \pm 54$} & \multirow{3}{*}{$0.40 \pm 0.01$} & \multirow{3}{*}{$-32.2 \pm 0.5$} & \multirow{3}{*}{--} & \multirow{3}{*}{--} \\
\hline & & Miglyol 182 & 150 & & & & & & & & & & & & \\
\hline & & Tween 60 & 100 & & & & & & & & & & & & \\
\hline \multirow{3}{*}{ LN37 } & \multirow{3}{*}{ NLC } & Precirol Ato 5 & 330 & \multirow{3}{*}{20} & \multirow{3}{*}{4.4} & \multirow{3}{*}{12000} & \multirow{3}{*}{120} & \multirow{3}{*}{70} & \multirow{3}{*}{15} & \multirow{3}{*}{4.4} & \multirow{3}{*}{$531 \pm 21$} & \multirow{3}{*}{$0.320 \pm 0.007$} & \multirow{3}{*}{$-29.4 \pm 0.8$} & \multirow{3}{*}{96.1} & \multirow{3}{*}{3.} \\
\hline & & $\begin{array}{l}\text { Miglyol } 182 \\
\text { Tween } 60\end{array}$ & $\begin{array}{l}150 \\
100\end{array}$ & & & & & & & & & & & & \\
\hline & & Oxaprozin & 20 & & & & & & & & & & & & \\
\hline \multirow{3}{*}{ LN38 } & \multirow{3}{*}{ NLC } & Precirol Ato 5 & 180 & & & & & & & & & & & & \\
\hline & & $\begin{array}{l}\text { Miglyol } 182 \\
\text { Tween } 60\end{array}$ & $\begin{array}{l}90 \\
60\end{array}$ & 10 & 8.8 & 7000 & 30 & 70 & 5 & -- & $340 \pm 8$ & $0.219 \pm 0.004$ & $-33.5 \pm 0.9$ & -- & -- \\
\hline & & Oxaprozin & 20 & & & & & & & & & & & & \\
\hline & & Precirol Ato 5 & 180 & & & & & & & & & & & & \\
\hline LN39 & NLC & $\begin{array}{l}\text { Oleic Acid } \\
\text { Tween } 60\end{array}$ & $\begin{array}{l}90 \\
60\end{array}$ & 10 & 8.8 & 7000 & 30 & 70 & 5 & -- & $425 \pm 10$ & $0.318 \pm 0.006$ & $-32.3 \pm 0.5$ & -- & -- \\
\hline & & Oxaprozin & 20 & & & & & & & & & & & & \\
\hline & & Precirol Ato 5 & 200 & & & & & & & & & & & & \\
\hline LN40 & NLC & Miglyol 182 & 90 & 10 & 4.4 & 7000 & 30 & 70 & 5 & 4.4 & $375 \pm 5$ & $0.19 \pm 0.01$ & -- & -- & -- \\
\hline & & Tween 60 & 60 & & & & & & & & & & & & \\
\hline & & Cetyl Palmitate & 270 & & & & & & & & & & & & \\
\hline LN41 & SLN & Tween 60 & 60 & 10 & 8.8 & 7000 & 30 & 70 & 5 & -- & $241 \pm 7$ & $0.121 \pm 0.005$ & $-61 \pm 3$ & 97.8 & 5.9 \\
\hline & & Oxaprozin & 20 & & & & & & & & & & & & \\
\hline LN42 & SLN & $\begin{array}{l}\text { Cetyl Palmitate } \\
\text { Tween } 60\end{array}$ & $\begin{array}{r}290 \\
60\end{array}$ & 10 & 8.8 & 7000 & 30 & 70 & 5 & -- & $264 \pm 1$ & $0.16 \pm 0.02$ & $-52.6 \pm 0.6$ & -- & -- \\
\hline & & Precirol Ato 5 & 180 & & & & & & & & & & & & \\
\hline LN43 & NLC & $\begin{array}{l}\text { Miglyol } 182 \\
\text { Tween } 60\end{array}$ & $\begin{array}{l}90 \\
60\end{array}$ & 10 & 4.4 & 7000 & 30 & 70 & 5 & 4.4 & $217 \pm 5$ & $0.120 \pm 0.004$ & $-44.7 \pm 0.9$ & 95.8 & 5.8 \\
\hline & & Oxaprozin & 20 & & & & & & & & & & & & \\
\hline
\end{tabular}




\begin{tabular}{|c|c|c|c|c|c|c|c|c|c|c|c|c|c|c|c|}
\hline \multirow{3}{*}{ LN44 } & \multirow{3}{*}{ NLC } & Precirol Ato 5 & 200 & \multirow{3}{*}{10} & \multirow{3}{*}{4.4} & \multirow{3}{*}{7000} & \multirow{3}{*}{30} & \multirow{3}{*}{70} & \multirow{3}{*}{5} & \multirow{3}{*}{4.4} & \multirow{3}{*}{$211.9 \pm 4$} & \multirow{3}{*}{$0.11 \pm 0.01$} & \multirow{3}{*}{$-55.6 \pm 0.6$} & \multirow{3}{*}{--} & \multirow{3}{*}{--} \\
\hline & & Miglyol 182 & 90 & & & & & & & & & & & & \\
\hline & & Tween 60 & 60 & & & & & & & & & & & & \\
\hline \multirow{3}{*}{ LN45 } & \multirow{3}{*}{ SLN } & Cetyl Palmitate & 260 & \multirow{3}{*}{10} & \multirow{3}{*}{8.8} & \multirow{3}{*}{7000} & \multirow{3}{*}{30} & \multirow{3}{*}{70} & \multirow{3}{*}{5} & \multirow{3}{*}{--} & \multirow{3}{*}{$244 \pm 4$} & \multirow{3}{*}{$0.07 \pm 0.01$} & \multirow{3}{*}{$-32.1 \pm 0.9$} & \multirow{3}{*}{98.9} & \multirow{3}{*}{9.} \\
\hline & & Tween 60 & 60 & & & & & & & & & & & & \\
\hline & & Oxaprozin & 30 & & & & & & & & & & & & \\
\hline \multirow{4}{*}{ LN46 } & \multirow{4}{*}{ NLC } & Precirol Ato 5 & 170 & & & & & & & & & & & & \\
\hline & & Miglyol 182 & 90 & 10 & 44 & 7000 & 30 & 70 & 5 & 44 & $215+1$ & $011+001$ & $281+0$ & 075 & 01 \\
\hline & & Tween 60 & 60 & & & & & & & & $210-1$ & $0.01-0.01$ & $20.1+0.0$ & & \\
\hline & & Oxaprozin & 30 & & & & & & & & & & & & \\
\hline & & Cetyl Palmitate & 266.7 & & & & & & & & & & & & \\
\hline IN47 & SIN & Tween 60 & 60 & 10 & 88 & 7000 & 30 & 70 & 5 & $\ldots$ & $250+3$ & $006+002$ & $-318+04$ & 952 & 58 \\
\hline & & Oxaprozin & 20 & & & & & & & & $200-1$ & & & & \\
\hline & & DSPE-PEG ${ }_{2000}-\mathrm{FA}$ & 3.3 & & & & & & & & & & & & \\
\hline & & Precirol Ato 5 & 176.7 & & & & & & & & & & & & \\
\hline & & Miglyol 182 & 90 & & & & & & & & & & & & \\
\hline LN48 & $\mathrm{NLC}$ & Tween 60 & 60 & 10 & 4.4 & 7000 & 30 & 70 & 5 & 4.4 & $211 \pm 3$ & $0.174 \pm 0.007$ & $-33 \pm 1$ & 83.6 & 5.1 \\
\hline & & Oxaprozin & 20 & & & & & & & & & & & & \\
\hline & & DSPE-PEG ${ }_{2000}-\mathrm{FA}$ & 3.3 & & & & & & & & & & & & \\
\hline & & Cetyl Palmitate & 263.4 & & & & & & & & & & & & \\
\hline $1 N 49$ & SIN & Tween 60 & 60 & 10 & 88 & 7000 & 30 & 70 & 5 & 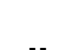 & $248+5$ & $006+00 ?$ & $-27+1$ & 040 & $57,-$ \\
\hline & & Oxaprozin & 20 & 10 & & & & & & & & & & & 0.1 \\
\hline & & DSPE-PEG ${ }_{2000}-\mathrm{FA}$ & 6.6 & & & & & & & & & & & & \\
\hline
\end{tabular}




\begin{tabular}{|c|c|c|c|c|c|c|c|c|c|c|c|c|c|c|c|}
\hline & & Precirol Ato 5 & 193.4 & & & & & & & & & & & & \\
\hline & & Miglyol 182 & 90 & & & & & & & & & & & & \\
\hline \multirow[t]{5}{*}{ LN50 } & NLC & Tween 60 & 60 & 10 & 4.4 & 7000 & 30 & 70 & 5 & 4.4 & $204 \pm 3$ & $0.17 \pm 0.02$ & $-32.5 \pm 0.7$ & 92.4 & 5.3 \\
\hline & & Oxaprozin & 20 & & & & & & & & & & & & \\
\hline & & DSPE-PEG ${ }_{2000}-\mathrm{FA}$ & 6.6 & & & & & & & & & & & & \\
\hline & & Precirol Ato 5 & 168 & & & & & & & & & & & & \\
\hline & & Miglyol 182 & 90 & & & & & & & & & & & & \\
\hline \multirow[t]{5}{*}{ LN51 } & NLC & Tween 60 & 60 & 10 & 4.4 & 7000 & 30 & 70 & 5 & 4.4 & $237 \pm 4$ & $0.185 \pm 0.007$ & $-29.3 \pm 0.7$ & 88.3 & 6.8 \\
\hline & & Oxaprozin & 25 & & & & & & & & & & & & \\
\hline & & DSPE-PEG ${ }_{2000}-\mathrm{FA}$ & 7 & & & & & & & & & & & & \\
\hline & & Precirol Ato 5 & 163 & & & & & & & & & & & & \\
\hline & & Miglyol 182 & 90 & & & & & & & & & & & & \\
\hline \multirow[t]{5}{*}{ LN52 } & NLC & Tween 60 & 60 & 10 & 4.4 & 7000 & 30 & 70 & 5 & 4.4 & $221 \pm 4$ & $0.169 \pm 0.005$ & $-35 \pm 1$ & 93.1 & 8.7 \\
\hline & & Oxaprozin & 30 & & & & & & & & & & & & \\
\hline & & DSPE-PEG ${ }_{2000}-F A$ & 7 & & & & & & & & & & & & \\
\hline & & Precirol Ato 5 & 170 & & & & & & & & & & & & \\
\hline & & Miglyol 182 & 90 & & & & & & & & & & & & \\
\hline \multirow[t]{5}{*}{ LN53 } & NLC & Tween 60 & 60 & 10 & 4.4 & 7000 & 30 & 70 & 5 & 4.4 & $251 \pm 11$ & $0.19 \pm 0.01$ & $-22.6 \pm 0.9$ & 84.1 & 7.6 \\
\hline & & Oxaprozin & 30 & & & & & & & & & & & & \\
\hline & & DSPE-PEG ${ }_{2000}-F A$ & 14 & & & & & & & & & & & & \\
\hline & & Precirol Ato 5 & 170 & & & & & & & & & & & & \\
\hline & & Miglyol 182 & 90 & & & & & & & & & & & & \\
\hline \multirow[t]{3}{*}{ LN54 } & NLC & Tween 60 & 60 & 10 & 4.4 & 7000 & 30 & 70 & 5 & 4.4 & $280 \pm 14$ & $0.16 \pm 0.01$ & $-23.1 \pm 0.5$ & 86.1 & 7.8 \\
\hline & & Oxaprozin & 30 & & & & & & & & & & & & \\
\hline & & DSPE-PEG ${ }_{2000}-F A$ & 10.5 & & & & & & & & & & & & \\
\hline
\end{tabular}




\begin{tabular}{|c|c|c|c|c|c|c|c|c|c|c|c|c|c|c|c|}
\hline \multirow{5}{*}{ LN55 } & \multirow{5}{*}{ NLC } & Precirol Ato 5 & 170 & \multirow{5}{*}{10} & \multirow{5}{*}{4.4} & \multirow{5}{*}{7000} & \multirow{5}{*}{30} & \multirow{5}{*}{70} & \multirow{5}{*}{5} & \multirow{4}{*}{4.4} & \multirow{5}{*}{$253 \pm 6$} & \multirow{5}{*}{$0.255 \pm 0.007$} & \multirow{5}{*}{$-31.3 \pm 0.6$} & \multirow{5}{*}{91.2} & \multirow{5}{*}{8.} \\
\hline & & Miglyol 182 & 90 & & & & & & & & & & & & \\
\hline & & Tween 60 & 60 & & & & & & & & & & & & \\
\hline & & Oxaprozin & 30 & & & & & & & & & & & & \\
\hline & & DSPE-PEG ${ }_{2000}-F A$ & 7 & & & & & & & & & & & & \\
\hline
\end{tabular}




\section{3 - Nanoparticles characterization}

In order to ensure that the developed formulations possessed the desired and necessary characteristics to allow a controlled and targeted oxaprozin release the lipid nanoparticle that resulted from the optimization process was then characterized along with its correspondent's placebo and non-functionalized particles.

The first indicator of a successful functionalization process and of the synthesis of a workable liquid suspension was visual confirmation. From Figure 4 we can verify the formation of a milky suspension characteristic of the ultra-sonication lipid nanoparticle synthesis method. These suspensions were of low viscosity which is essential to its use, having the formulations with oxaprozin a slightly higher viscosity. It is also possible to notice colour change in the functionalized nanoparticles when compared with the non-functionalized correspondents. This colour alteration is the first indicative of a successful functionalization of the formulations. Oxaprozin deposits were not visible however small DSPE-PEG $2000-F A$ deposits were visible tending to disappear over time and with agitation which may indicate suspension stabilization over time.

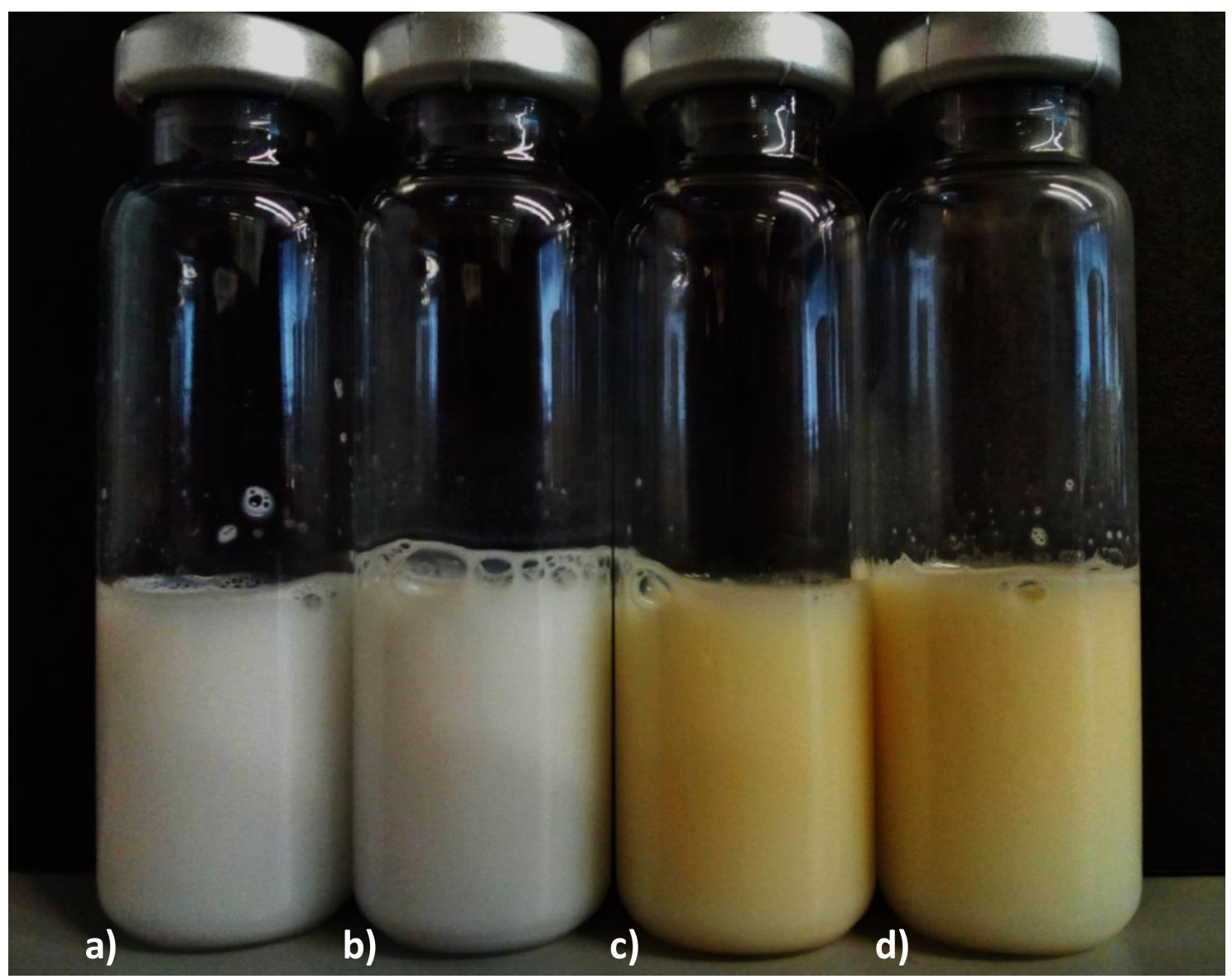

Figure 4: Lipid nanoparticles synthesised by emulsification-sonication: a) Non-functionalized NLCs placebo, b) Non-functionalized NLCs + Oxa, c) Functionalized NLCS Placebo, d) Functionalized NLCs + Oxa. 
Nanoparticles diameter, polydispersity, zeta potential, encaplsulation efficiency and loading capacity were also evaluated and it is summarized on the Table 4.

Table 4: Physicochemical properties of the developed LNs. Mean effective diameter, polydispersity, zeta potential, encapsulation efficiency and loading capacity of placebo and oxaprozin non-functionalized and folate functionalized LNs. Values represent mean $\pm S D(n=3)$; a) $P<0.05$ relatively to the correspondent NLCs placebo formulation; b) $\mathrm{P}<0.05$ relatively to the non-functionalized NLCs formulation.

\begin{tabular}{ccccc}
\cline { 2 - 4 } & $\begin{array}{c}\text { Non-funcionalized } \\
\text { NLCs Placebo }\end{array}$ & $\begin{array}{c}\text { Non-funcionalized } \\
\text { NLCs }+ \text { Oxa }\end{array}$ & $\begin{array}{c}\text { Funcionalized } \\
\text { NLCs Placebo }\end{array}$ & $\begin{array}{c}\text { Funcionalized } \\
\text { NLCs }+ \text { Oxa }\end{array}$ \\
\hline Diameter $(\mathrm{nm})$ & $216 \pm 23$ & $289 \pm 27^{\mathrm{a}}$ & $234 \pm 24$ & $281 \pm 25^{\mathrm{a}}$ \\
\hline Polydispersity & $0.28 \pm 0.04$ & $0.17 \pm 0.03$ & $0.23 \pm 0.05^{\mathrm{b}}$ & $0.14 \pm 0.03$ \\
\hline $\begin{array}{c}\text { Zeta Potential } \\
(\mathrm{mV})\end{array}$ & $-41 \pm 2$ & $-44 \pm 2$ & $-41 \pm 2$ & $-42 \pm 2$ \\
\hline $\begin{array}{c}\text { Encapsulation } \\
\text { Efficiency (\%) }\end{array}$ & - & $97.6 \pm 0.3$ & - & $95.1 \pm 0.5^{\mathrm{b}}$ \\
\hline $\begin{array}{c}\text { Loading Capacity } \\
(\%)\end{array}$ & - & $9.1 \pm 0.3$ & - & $8.7 \pm 0.5^{\mathrm{b}}$ \\
\hline \hline
\end{tabular}

Considering the size and polydispersity of our nanoparticles, it is necessary to have into consideration that they were obtained using filtered nanoparticles. The filtration process was necessary in order to reduce the deviating effect that bigger particles cause in the diameter reading process, this also allowed to ensure that even the smallest particles had the desired diameter. From the obtained data Table 4 one can conclude that the NLCs diameters were all greater than $200 \mathrm{~nm}$, meaning that our nanoparticles can be recognized be the macrophages as desired $[77,79]$. Despite the fact that the functionalization process did not interfered with the diameter of the nanoparticles, the oxaprozin encapsulation leads to the formation of nanoparticles of large diameter than the corresponding placebo which can be a prove that the oxaprozin is in fact being encapsulated and may help to stabilize the lipids aggregates during the synthesis.

The polydispersity of the formulations were around 0.2 , meaning that formulations are constituted by a well-defined and almost monodisperse population of nanoparticles with low variability typical of LNs produced using the high shear homogenization and ultrasound method [129]. These values also suggest a uniform functionalization process that leads to a small decrease on the polydispersity.

Zeta potential of the particles that constitute the formulations is greater in modulus to 40 $\mathrm{mV}$ ensuring the stability of our formulations and revealing that they do not tend to form aggregates since they are superior to the reference value of $|30| \mathrm{mV}[123,124]$. Oxaprozin encapsulation and the functionalization process did not interfered with the zeta potential values and consequently with the stability of the formulations.

The encapsulation efficiency and consequently the loading capacity are influenced by the functionalization process, leading to slightly lower values of these parameters. However the obtained values are good and very satisfactory revealing the high encapsulation efficiency and loading capacity of these nanoparticles.

Transmission electron microscopy (TEM) was also used to analyse the formulations. Transmission electron micrographs (Figure 5) revealed the spherical shape of the nanoparticles and that the encapsulation and functionalization process did not to considerably affect the particles shape. The micrographs shown also particles with sizes slightly different from those obtained by DLS. Though, most of the particles, primarily from the functionalized oxaprozin loaded NLC (the formulation of interest) demonstrated sizes 
superior to $200 \mathrm{~nm}$ which is the minimum size pretended for our particles. Diameter differences between TEM and DLS analysis can be justified by the fact that DLS analyses the hydrodynamic radius. Moreover DLS provides a mean size of the populations present in the formulation while TEM provides a direct size of the particles.
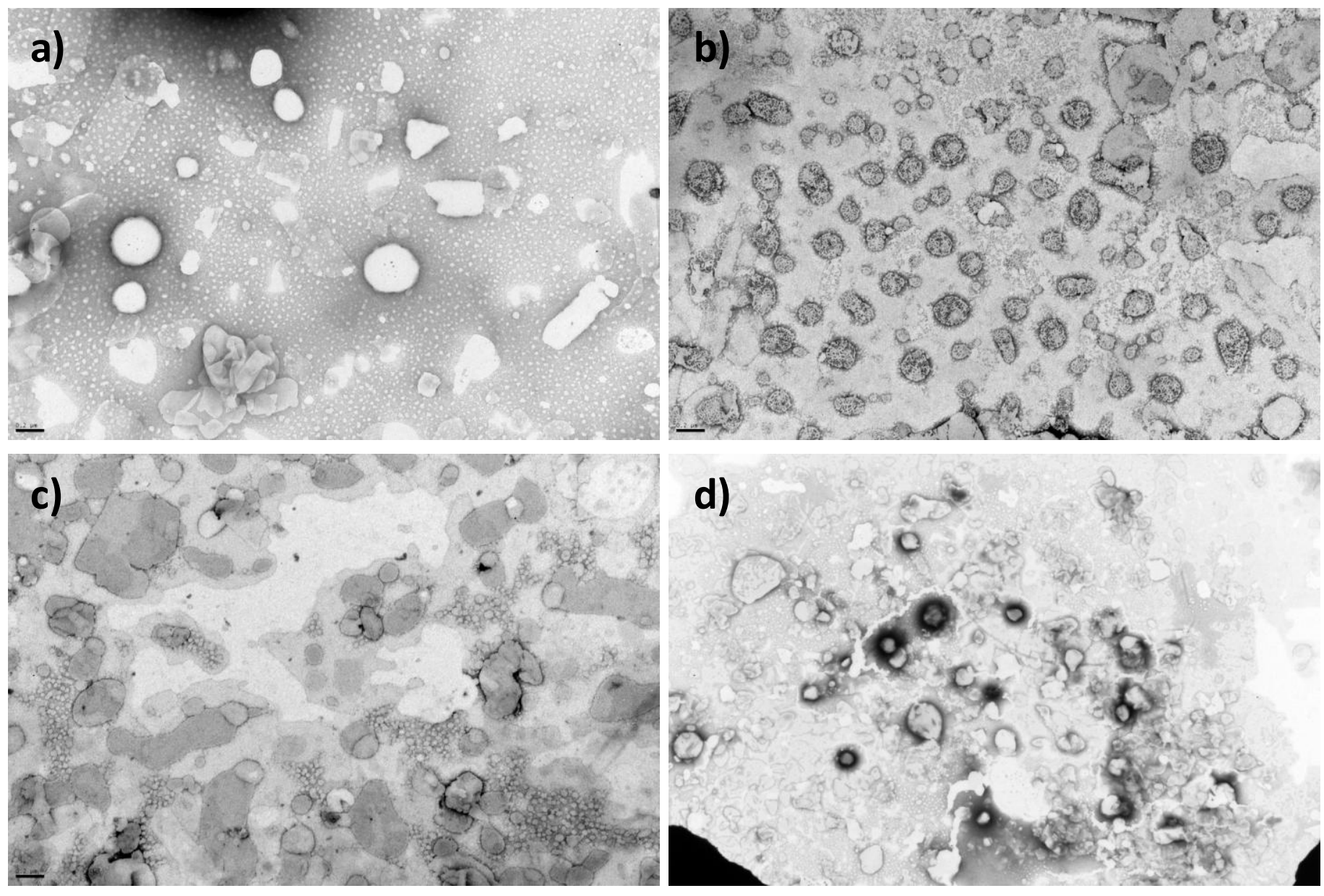

Figure 5: TEM micographs: a) Non-functionalized NLCs placebo, Magnification 40000X. b) Non-functionalized NLCs + Oxa, Magnification 40000X. c) Functionalized NLCs Placebo, Magnification 40000X. d) Functionalized NLCs + Oxa, Magnification 12000 .

UV-Vis spectophotometric analysis of the functionalized LNs after being destroyed revealed a peak on the Placebo $L N$ similar to those found on free functionalization agent, DSPE-PEG ${ }_{2000}-\mathrm{FA}$, (Figure 6) on the other hand in oxaprozin loaded LNs due to the presence of the oxaprozin with a broad peak near the functionalization agent peak one cannot clearly identify the functionalization agent peak (Figure 7). However the qualitative analysis can prove the presence of the functionalization agent on the LNs. 


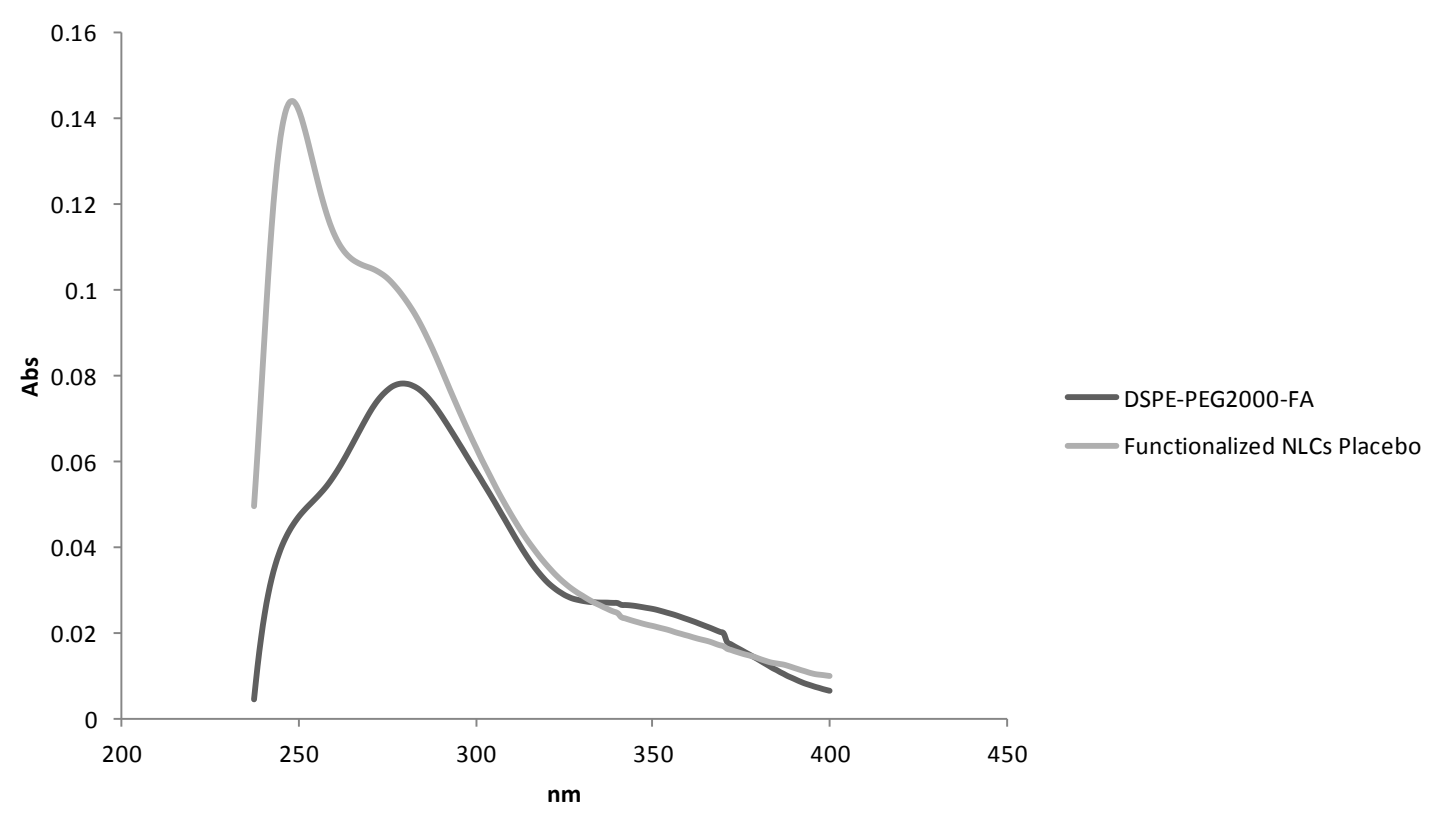

Figure 6: UV-Vis analysis of the functionalization process in Functionalized NLCs placebo.



Figure 7: UV-Vis analysis of the functionalization process on Functionalized NLCS + oxaprozin.

\section{4 - In vitro release studies}

The in vitro oxaprozin release study was designed in order to simulate the lipid nanoparticles path through the body after oral administration. Particles were placed at body temperature $\left(37^{\circ} \mathrm{C}\right)$ in gastric medium for 3 hours and then 4 hours in intestinal medium in order to simulate the gastrointestinal transit. Then the particles were placed some in physiologic medium in order to simulate particles that never reach the inflamed region and stood in circulation, some in inflammatory medium to simulate particles that immediately accumulate in inflamed regions and some $18 \mathrm{~h}$ hours in physiologic medium and then in 
inflammatory medium to reproduce the path of particles trough the blood and its later placement in inflamed regions.

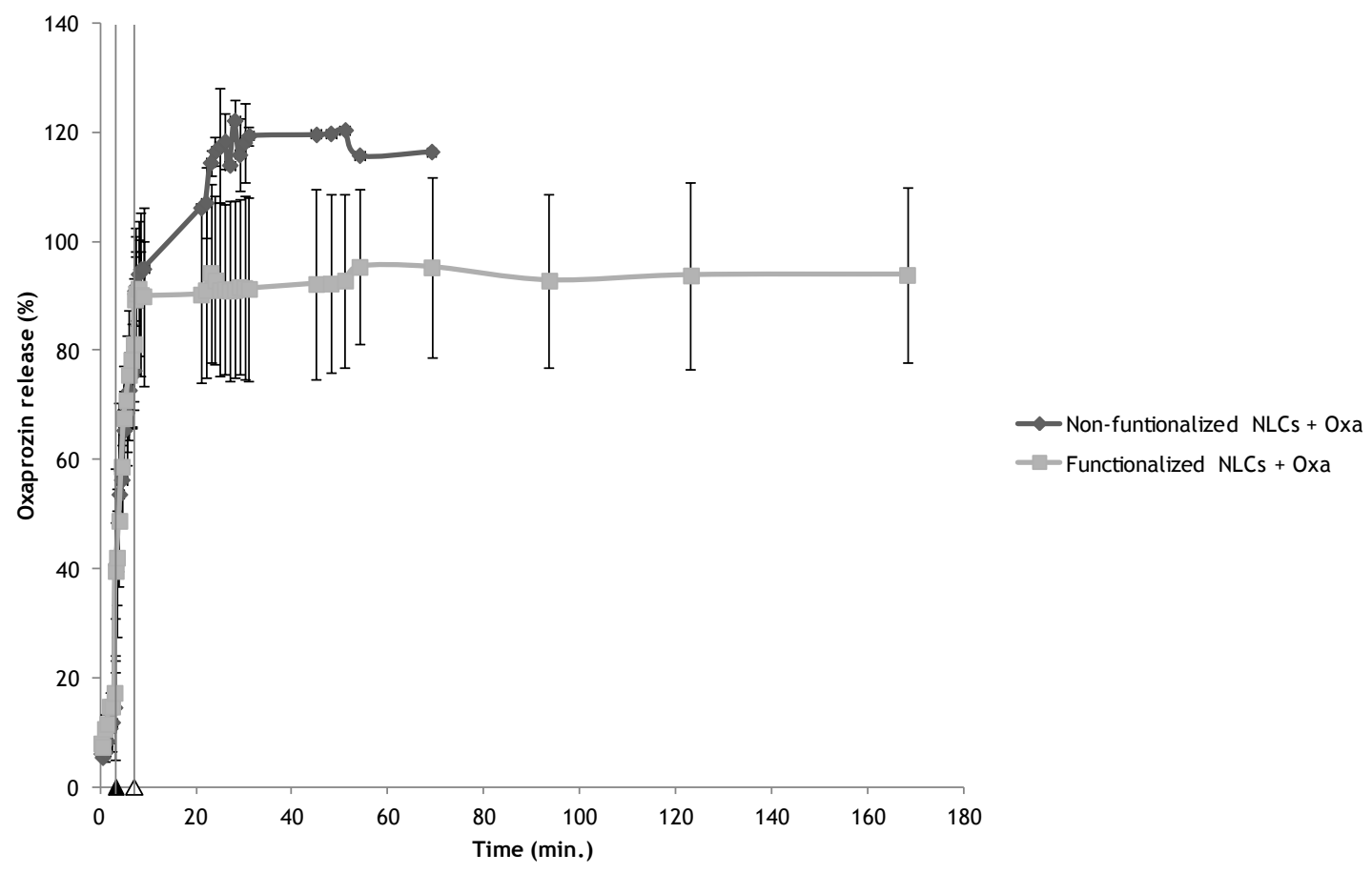

Figure 8: Oxaprozin release in gastric, intestinal and physiologic media (vertical lines represent media alterations).



Figure 9: Oxaprozin release in gastric, intestinal and inflammatory media (vertical lines represent media alterations). 


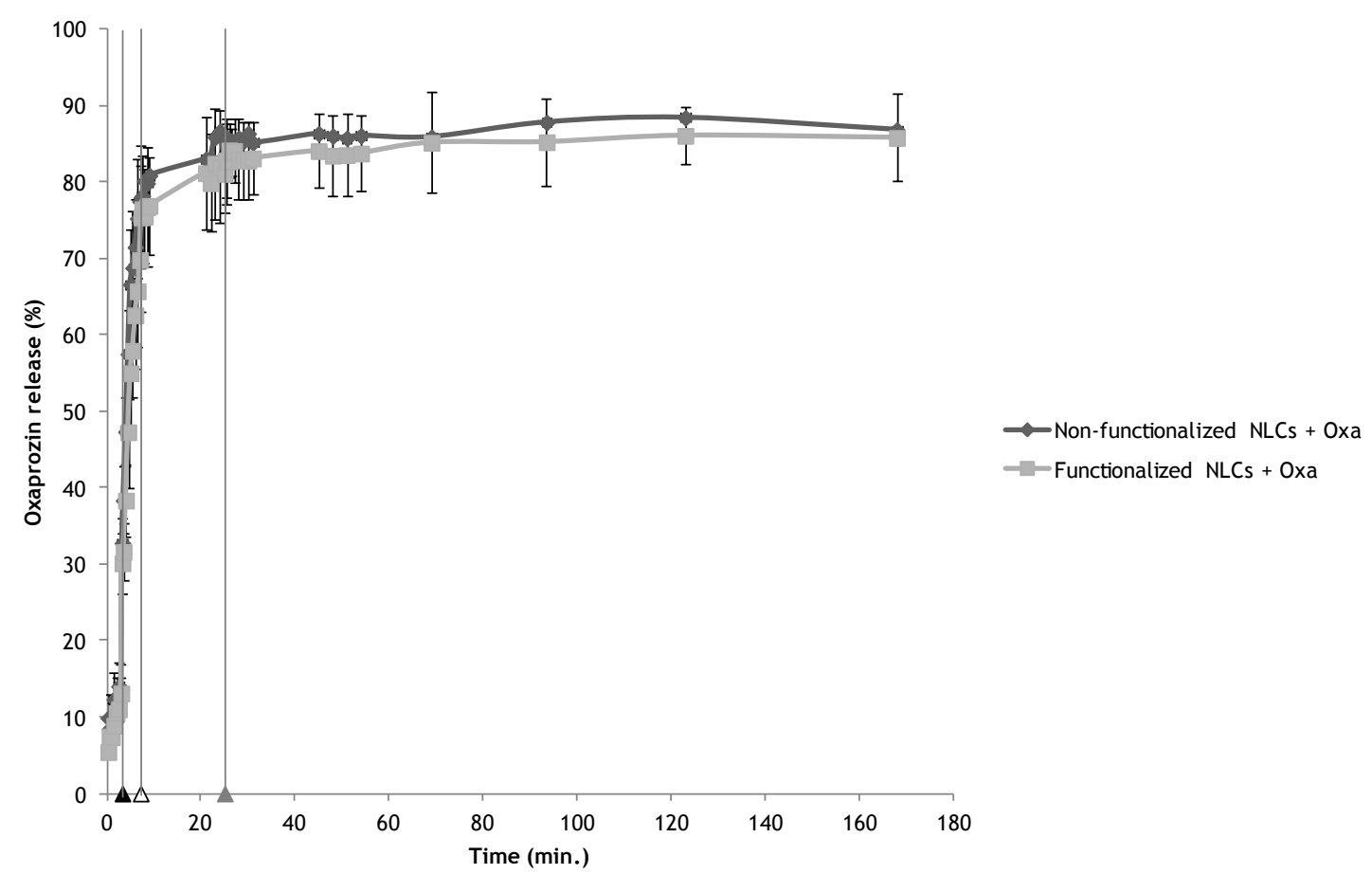

Figure 10: Oxaprozin release in gastric, intestinal, physiological and inflammatory media (vertical lines represent media alterations).

Analysing the data (Figure 8, 9 and 10) is possible to observe that for every condition the release seems to be identical for both functionalized and non-functionalized particles revealing that the functionalization process does not interfere with the in vitro release profile. Non-functionalized oxaprozin loaded NLCs in physiological medium escape this tendency, however is also evident that the profile is unstable and the values reveal amounts of released oxaprozin superiors to those used, whereupon it is necessary to repeat the experiment to take further conclusions. As desired only a small amount of oxaprozin is released in gastric medium, less than 20\%, which may help reduce oxaprozin side effects on the gastric mucosa. Most of the encapsulated oxaprozin is released on the intestinal medium leaving only about $20 \%$ of the encapsulated oxaprozin to be released on physiologic and inflammatory media. The oxaprozin released on the intestine will be absorbed by the mucosa and distributed throughout the body similarly to the free oxaprozin traditional administration. These results also indicate that the functionalization may be unnecessary or at least will play a secondary role since most of the encapsulate oxaprozin is released on the intestinal medium leaving only about $20 \%$ of the encapsulated oxaprozin to be delivery through macrophage targeting on inflamed regions. One can also verify that approximately $10 \%$ of the encapsulated oxaprozin remains entrapped on the crystalline matrix of the lipid nanoparticles.

\section{5 - Effect of LNs on cell viability and cytotoxicity}

In order to evaluate the cytotoxicity of the designed nanoparticles in the intestinal mucosa MTT and LDH assays were performed using Caco-2 cell line (Figure 11). On both tests the assayed values of cells incubated with the nanoparticles with different oxaprozin 
concentrations were compared with the mean of positive controls: culture medium and TX100 for the MTT and LDH assays, respectively.

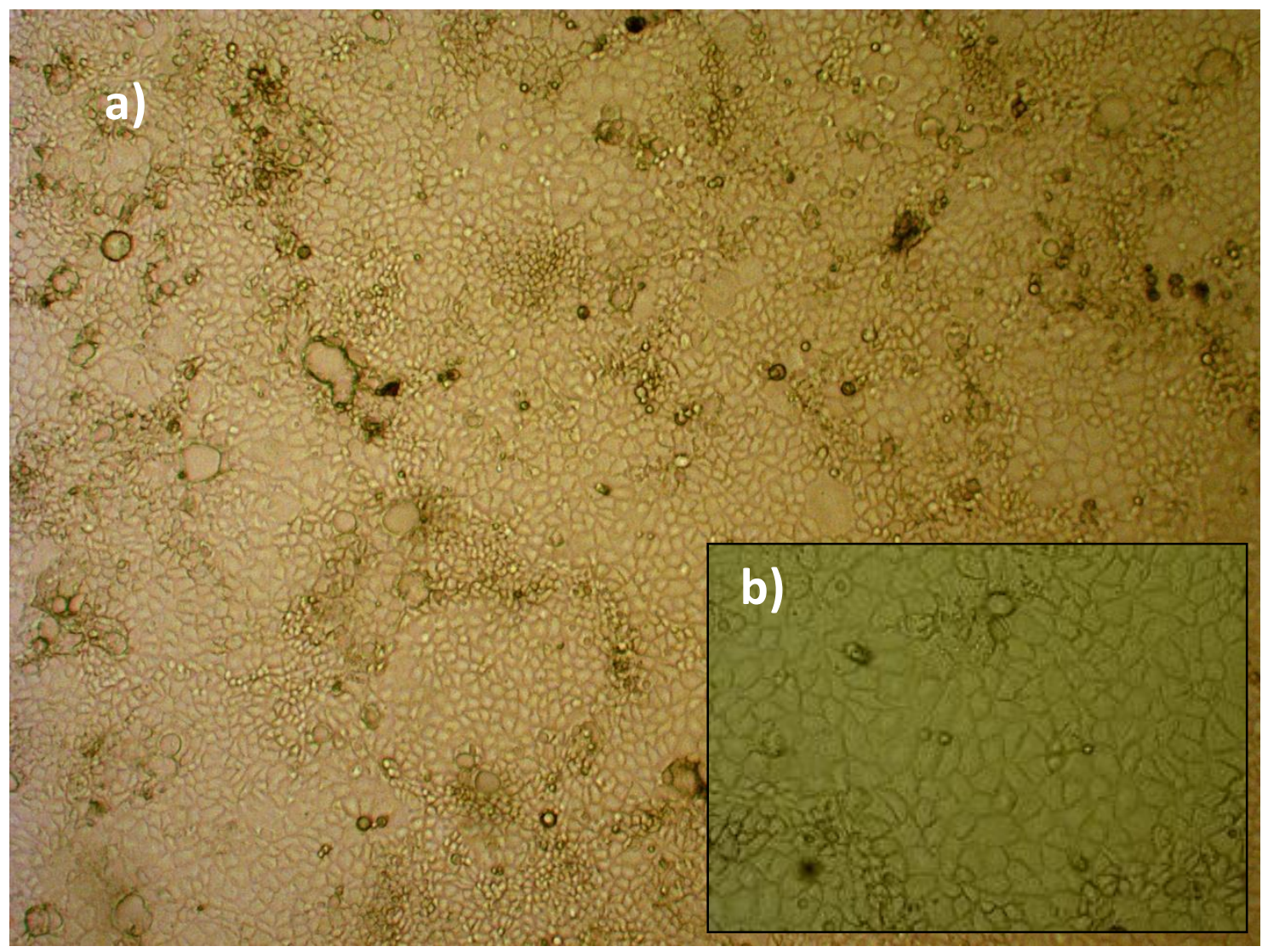

Figure 11: General morphology of confluent Caco-2 cells by inverted microscope observation: a) Magnification 40X, b) Magnification 100X.

From the MTT assay Figure 12 one can observe that free oxaprozin at these concentrations does not reveal a cytotoxic effect being the cell viability around $100 \%$ contrarily to the negative control TX-100 with close to $0 \%$ cell viability, revealing high cytotoxicity, as expected. The comparison between the different nanoparticles and the different oxaprozin concentrations reveals that only the highest concentrations tested (500 $\mu \mathrm{M}$ and $1000 \mu \mathrm{M}$ oxaprozin concentration) present, up to some extent, cytotoxicity to the tested cell line in all the LNs formulations. Nevertheless, since free oxaprozin does not present cytotoxicity at these concentrations and that the correspondent amount of placebo formulations for these concentrations reveals also cytotoxicity, one can conclude that the cytotoxic effect at these concentrations could not result from the oxaprozin but from the LNs themselves, due to their physico-chemical characteristics, the presence of high lipid contents or improved oxaprozin uptake promoted by the LNs. 


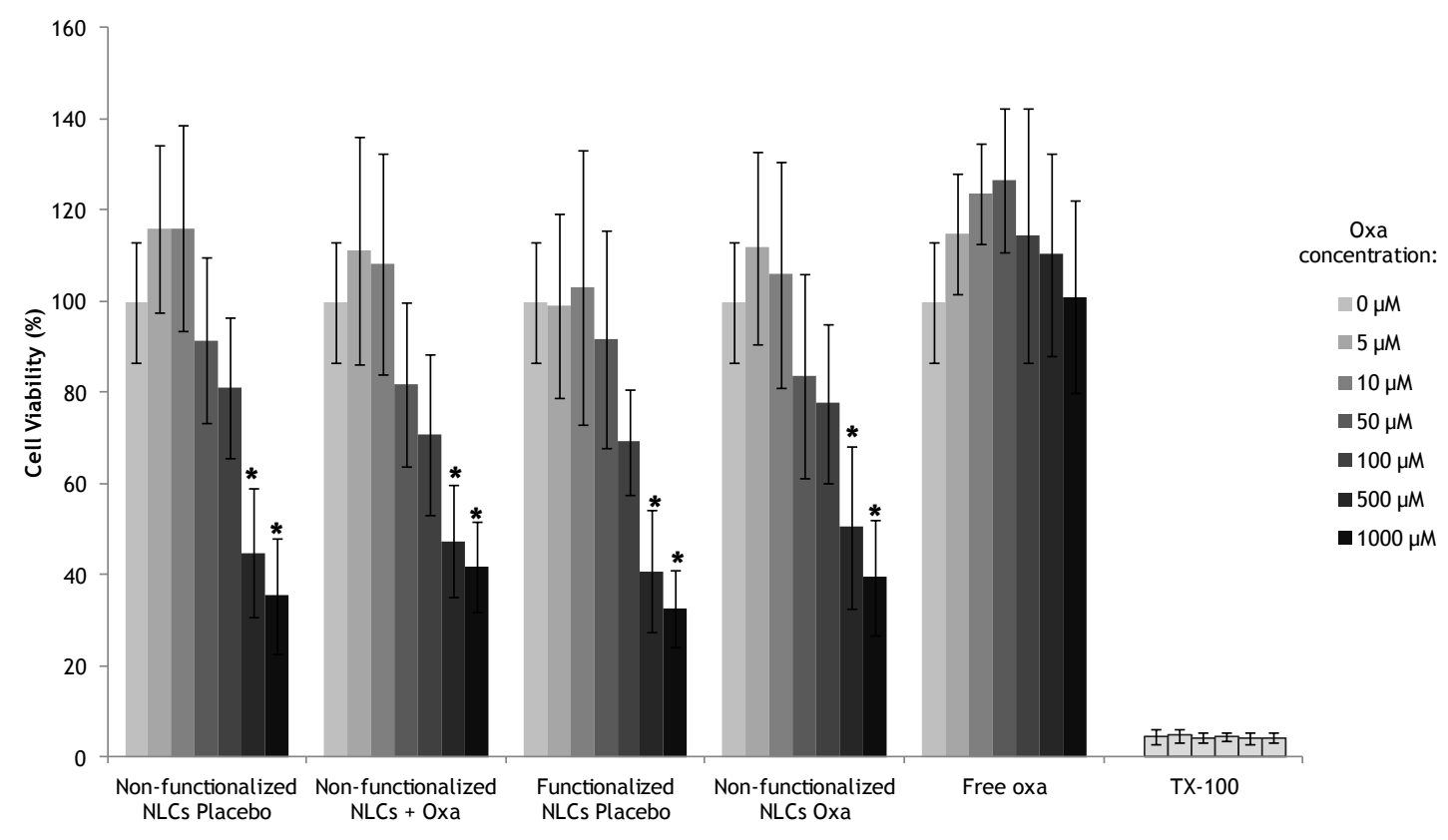

Figure 13: Caco-2 cell viability assessed by MTT assay as a function of the different formulations and concentrations tested $(5,10,50,100,500,1000 \mu M)$. Values represent mean $\pm S D\left(n=7,{ }^{*} P<0.05\right.$ relatively to the mean positive control).

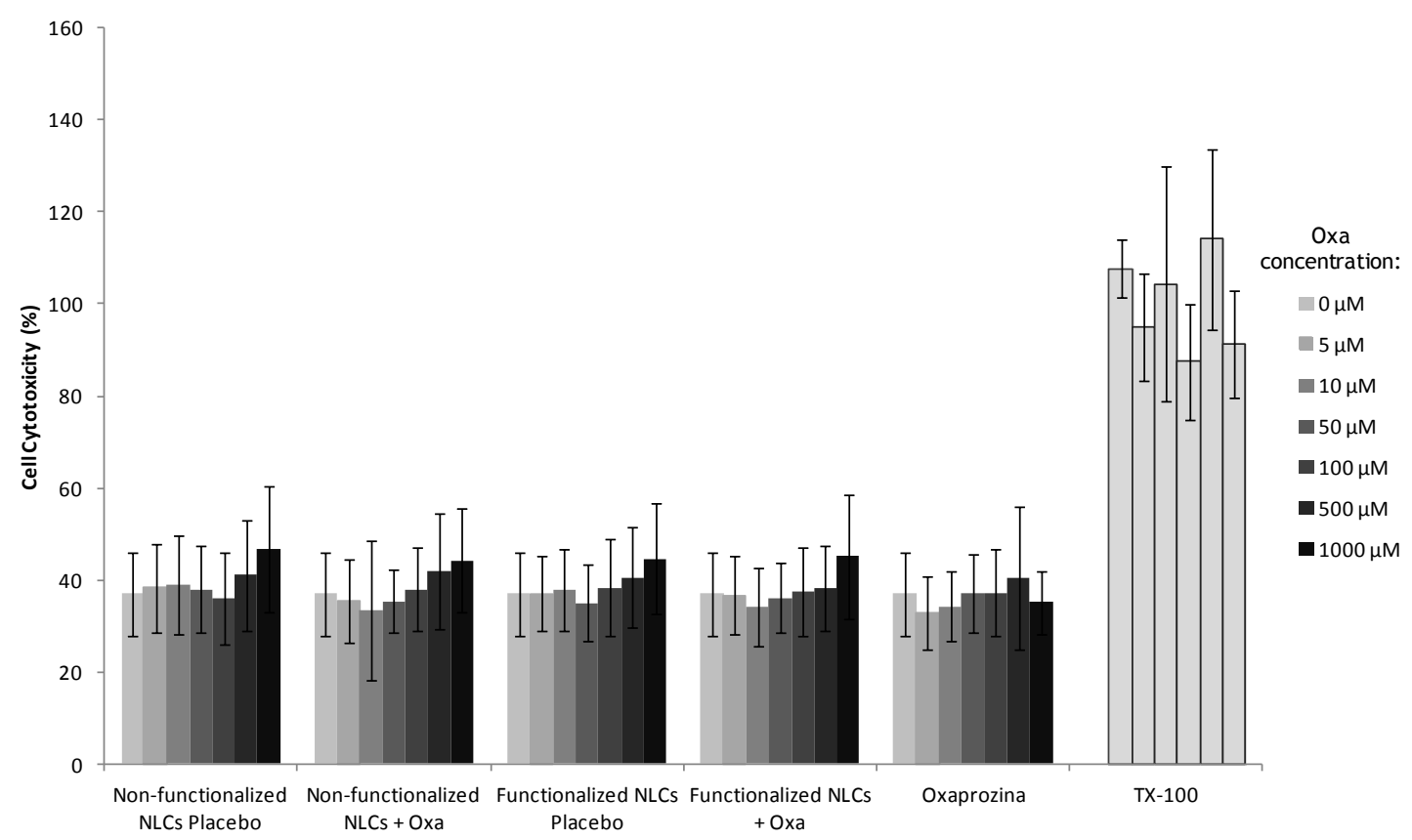

Figure 12: Caco-2 cell cytotoxicity assessed by LDH assay as a function of the different formulations and concentrations tested $(5,10,50,100,500,1000 \mu \mathrm{M})$. Values represent mean $\pm S D\left(n=7,{ }^{*} P<0.05\right.$ relatively to the mean positive control).

LDH assay (Figure 13) reveals that TX-100 cell cytotoxicity effect results primarily from membrane disruption as expected and that for cells treated only with culture medium, cell death due to membrane disruption corresponds to roughly $40 \%$. Analysing the data one can observe that this last value is equal to those obtained in the cells incubated with LNs for all the oxaprozin concentrations and correspondent placebos. Therefore, the cytotoxicity of LNs revealed at higher oxaprozin concentrations from the MTT assay does not result from 
membrane disruption which can corroborate the previous hypothesis in which cytotoxicity results from high oxaprozin uptake due to its encapsulation in LNs.

From MTT and LDH assays one can conclude that the highest concentration of loaded oxaprozin that one can use without interfere with cellular viability is $100 \mu \mathrm{M}$ for both functionalized and non-functionalized LNs and their correspondent placebos. This value goes along with a previous study in which this oxaprozin concentration was also used for oxaprozin combined with cyclodextrin, chitosan and bile components in Caco-2 cell permeability assay without cellular cytotoxicity being revealed [130].

\section{6 - Cell permeability studies}

In order to perform permeability studies, cells were seeded in the transwell inserts on the polycarbonate membranes. The monolayer development was followed by inverted microscopy and by transepithelial electrical resistance until the $21^{\text {st }}$ day. TEER values obtained at this time were superior than $200 \Omega . \mathrm{cm}^{2}$ meaning that the monolayer was mimicking the intestinal endothelium.
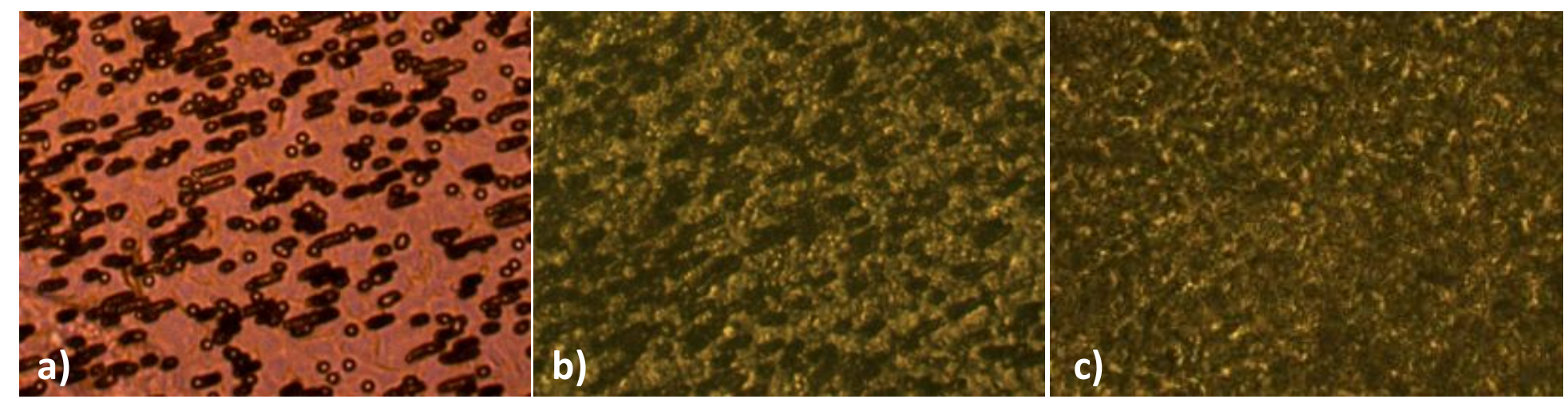

Figure 14: Monolayer formation on the Transwell inserts along time. a) Monolayer at day 0 , Magnification 400X, b) Monolayer at day 21, Magnification 400X, c) Monolayer at day 21, Magnification 200X.

From Figure 14 one can clearly see the membrane pores at day 0 (Figure 14.a)) and a decrease on its visibility at day 21 (Figure 14.b)) when compared to the initial time. On Figure 14.c) one can see the cells that form the monolayer that covers the inserts membrane.

Oxaprozin intestinal mucosa permeability was evaluated using the biorelevant medium that simulated fasted (FaSSIF) state and also PBS the reference medium.

Figure 15 illustrates oxaprozin permeability profile in FaSSIF medium and it is possible to observe that for all LNs tested there is at least $20 \%$ of permeation after 4 hours of assay. The Table (Figure 15) summarizes the apparent permeability coefficient (Papp) and for all the formulations Papp is around $10^{-5} \mathrm{~cm} \cdot \mathrm{s}^{-1}$ after 4 hours. LNs permeability profile is similar to the free oxaprozin profile revealing that oxaprozin encapsulation do not interfere so much with its permeability through the intestinal mucosa and also that the functionalization process do not affect the permeability of the LNs. 


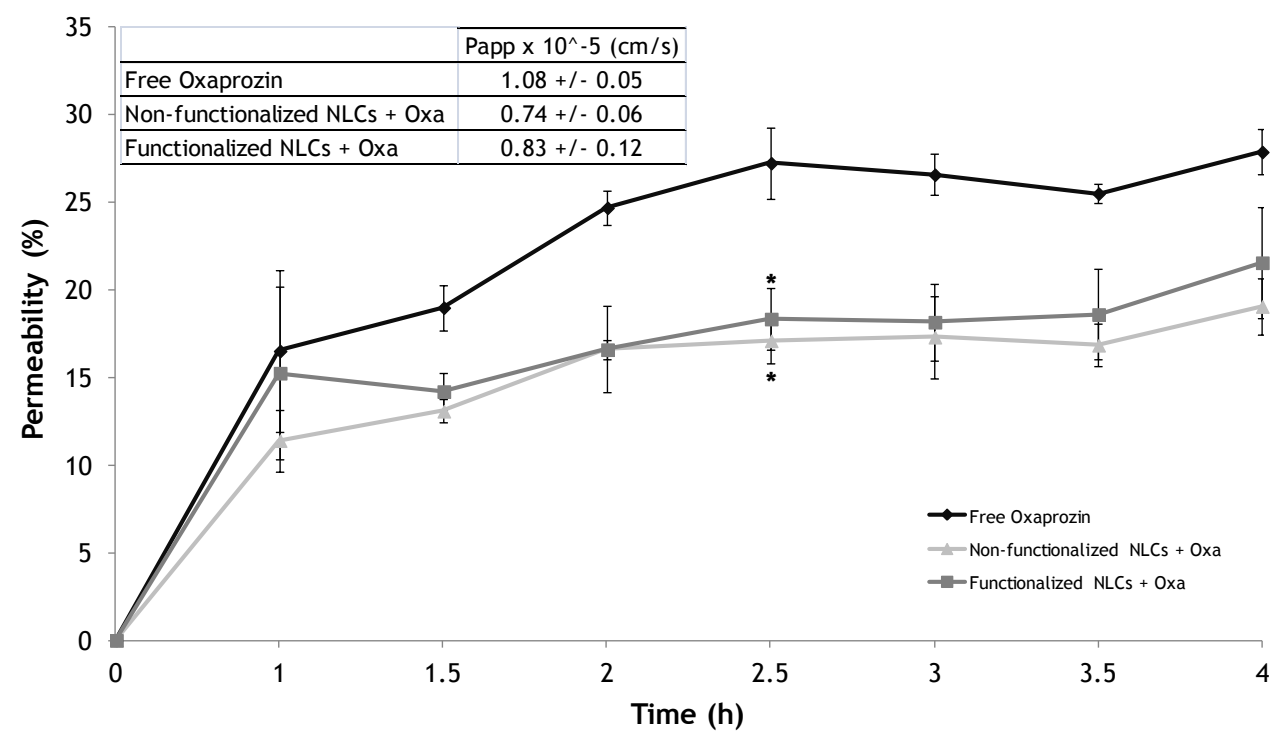

Figure 16: Oxaprozin permeability along the time and final apparent permeability (Table) for free oxaprozin and functionalized and non functionalized oxaprozin NLCS in FaSSIF. Values represent mean $\pm S D ; N \geq 2,{ }^{*} P<0.05$ comparatively to free oxaprozin.

In the reference medium (PBS) the permeability values are greater than in the FaSSIF medium reaching almost $40 \%$ of oxaprozin permeation for all samples tested (Figure16). One can notice that the functionalized formulation has a slow initial permeation profile through the monolayer compared to free oxaprozin and non-functionalized formulation. However in the end of the assay there are no significant differences between the formulations and free oxaprozin, reaching the apparent permeability coefficients values around $1.5 \times 10^{-5} \mathrm{~cm} . \mathrm{s}^{-1}$ (Table from Figure 16). Oxaprozin Papp value is of the same order of the ones presented in the literature for oxaprozin in HBSS $\left(2.3 \times 10^{-5} \mathrm{~cm} . \mathrm{s}^{-1}\right)$. The difference between these values may be justified by the small difference between media and the adjust of $\mathrm{pH}$ to 5.5 which promote an increase of permeation in relation to our value [130].



Figure 15: Oxaprozin permeability along the time and final apparent permeability (Table) for free oxaprozin and functionalized and non-functionalized oxaprozin NLCs in PBS. Values represent mean \pm SD; $N \geq 2,{ }^{*} \mathrm{P}<0.05$ comparatively to free oxaprozin. 
TEER values along the 4 hour assay were also followed and the mean medium size at each time compared to the optimal reference value of $200 \Omega . \mathrm{cm}^{2}$. For the different media used one could notice a decrease in this parameter $34 \%$ for FaSSIF and $28 \%$ for PBS, which is reasonable and is natural that occurs due to monolayer hydration by the presence of lipids and due to the LNs passage that slightly reduces the monolayer electrical resistance. 


\section{Chapter 4}

\section{Conclusions}

NSAIDs are effective anti-inflammatory, anti-pyretic and analgesic drugs and are of great importance for the treatment of chronic inflammatory conditions.

As a NSAID, oxaprozin is a non-selective COX inhibitor drug and possess strong antiinflammatory, antipyretic and analgesic effects, thus it may be used in the treatment of chronic inflammatory conditions. However, for that, its physicochemical properties should be improved in order to minimize its side effects, which can be achieved by the development of drug delivery systems. Nevertheless, the NSAIDs delivery systems developed to the date have still many drawbacks and thereby none of them is yet in the market.

Lipid Nanoparticles are widely used and allow an effective drug packaging and targeted delivery, improving drug characteristics and avoiding some of their side effects.

Oral administration is one of the most used and effective administration routes despite all the physical and chemical barriers that can degrade drugs and particles and diminish their uptake reducing drug effectiveness. However particles can be designed in order to overtake these barriers promoting the drug uptake and protection.

Therefore the creation of a folate functionalized oxaprozin loaded lipid nanoparticle may be an effective system able to deliver by oral route oxaprozin in specific locations avoiding these NSAID side effects and improving its chemical and physical characteristics.

In this work one attempted to create this nanosystem for oxaprozin delivery resulting on the creation of a DSPE-PEG 2000 -FA functionalized or non-functionalized Precirol Ato5, Miglyol 182, Tween 60 nanostuctured lipid carrier loaded with oxaprozin as final formulations. Formulation of these particles leads to the creation of size homogeneous suspensions with polydispersity around 0.150 and mean diameters around $285 \mathrm{~nm}$. Formulations zeta potential was superior to $-40 \mathrm{mV}$, suggesting a stable formulation. Oxaprozin encapsulation efficiency was high (bigger than 95\%) leading to a high loading capacity of the system (close to 9\%). Particles seemed to be spherical under TEM analysis with sizes slightly superiors to those obtained by DLS analysis.

The in vitro release study revealed that the designed nanosystem hold low oxaprozin release in simulated gastric fluid being the great majority released in simulated intestinal fluid leaving only a small amount of oxaprozin to be released on physiological and inflammatory medium (20\%). One can also verify that only a small amount of oxaprozin (less than $10 \%$ ) remains entrapped on the $L N$ matrix at the end of the study.

MTT and LDH assays on Caco-2 cells revealed LN cytotoxicity only for high oxaprozin concentrations being $100 \mu \mathrm{M}$ the highest oxaprozin concentration that can be used in order to the $L N$ do not have a cytotoxic effect.

Permeability studies in Caco-2 cell lines revealed that the final formulation does not reduced oxaprozin permeability through a monolayer of Caco- 2 cells simulating the intestinal endothelium. In fact, functionalized particles revealed permeability along time similar to the 
free oxaprozin and a similar apparent permeability both in PBS and simulated fasted intestinal fluid.

The designed particles and the obtained results suggest that this formulation enclose a huge potential for oxaprozin oral administration with potentially less significant gastric side effects, since the great majority of the entrapped oxaprozin is only released on the intestine, avoiding this way its contact with the gastric mucosa and its negative effects on it. The functionalization process seems to not interfere with the particles characteristics however since oxaprozin is mainly released in the intestine its role is secondary because only a small amount of oxaprozin remains on the particles to be released on the inflamed areas.

Despite the obtained results further tests and optimization of these particles are still needed and conducted until these nanosystems become a therapeutic reality. 


\section{Chapter 5}

\section{Future work}

For future work, lipid nanoparticles functionalization yield needs to be assessed in order to evaluate the efficiency of the functionalization process which can be achieved by nuclear Magnetic Resonance (NMR) spectroscopy.

Stability studies along the time need also to be conducted in order to define the LNs shelf-time. It is also important to define a lyophilisation protocol that does not compromise the LNs characteristics.

Cytotoxic studies must also be done in different cell lines (gastric, cardiac and macrophages) in order to verify that the LNs effectively reduce oxaprozin cytotoxicity and consequently its side effects.

Macrophages LNs uptake must also the analysed in order to verify if the functionalization process is effectively augmenting the LNs uptake by macrophages leading to an effective active targeting of the macrophages.

Ultimately, the efficacy of the designed nanosytem must be tested in vivo in healthy and disease animal model. 
-This page was intentionally left blank- 


\section{References}

1. Atchison, J.W., C.M. Herndon, and E. Rusie, NSAIDs for musculoskeletal pain management:current perspectives and novel strategies to improve safety. J Manag Care Pharm, 2013. 19(9 Suppl A): p. S3-19.

2. Ahmed, A., An overview of inflammation: mechanism and consequences. Frontiers in Biology, 2011. 6(4): p. 274-281.

3. Calder, P.C., et al., Inflammatory disease processes and interactions with nutrition. Br J Nutr, 2009. 101 Suppl 1: p. S1-45.

4. Cone, J.B., Inflammation. The American Journal of Surgery, 2001. 182(6): p. 558562.

5. Medzhitov, R., Inflammation 2010: New Adventures of an Old Flame. Cell, 2010. 140(6): p. 771-776.

6. Liantonio, J.J. and B.B. Simmons, NSAIDs and the Geriatric Patient: A Cautionary Tale. Clinical Geriatrics, 2013. 21(5).

7. Albishri, J., NSAIDs and hypertension. Anaesthesia, Pain \& Intensive Care, 2013. 17(2): p. 171-173.

8. Tomisato, W., et al., Role of direct cytotoxic effects of NSAIDs in the induction of gastric lesions. Biochem Pharmacol, 2004. 67(3): p. 575-85.

9. Vane, J.R. and R.M. Botting, Mechanism of action of nonsteroidal anti-inflammatory drugs. Am J Med, 1998. 104(3A): p. 2S-8S; discussion 21S-22S.

10. Turvey, S.E. and D.H. Broide, Innate immunity. Journal of Allergy and Clinical Immunology, 2010. 125(2, Supplement 2): p. S24-S32.

11. Parham, P., The Immune System, Third Edition2009: Taylor \& Francis Group.

12. Moilanen, E., Two Faces of Inflammation: An Immunopharmacological View. Basic \& Clinical Pharmacology \& Toxicology, 2014. 114(1): p. 2-6.

13. Beutler, B., Innate immunity: an overview. Molecular Immunology, 2004. 40(12): p. 845-859.

14. Medzhitov, R., Origin and physiological roles of inflammation. Nature, 2008. 454(7203): p. 428-435.

15. Pecchi, E., et al., Prostaglandins and sickness behavior: Old story, new insights. Physiology \& Behavior, 2009. 97(3-4): p. 279-292.

16. Kean, W.F. and W.W. Buchanan, The use of NSAIDs in rheumatic disorders 2005: a global perspective. Inflammopharmacology, 2005. 13(4): p. 343-70.

17. Sikiric, P., et al., Toxicity by NSAIDs. Counteraction by stable gastric pentadecapeptide BPC 157. Curr Pharm Des, 2013. 19(1): p. 76-83.

18. Bruno, A., S. Tacconelli, and P. Patrignani, Variability in the Response to NonSteroidal Anti-Inflammatory Drugs: Mechanisms and Perspectives. Basic \& Clinical Pharmacology \& Toxicology, 2014. 114(1): p. 56-63.

19. Knights, K.M., A.A. Mangoni, and J.O. Miners, Defining the COX inhibitor selectivity of NSAIDs: implications for understanding toxicity. Expert Rev Clin Pharmacol, 2010. 3(6): p. 769-76.

20. Vane, J.R., Introduction: mechanism of action of NSAIDs. Br J Rheumatol, 1996. 35 Suppl 1: p. 1-3.

21. Funk, C.D., Prostaglandins and Leukotrienes: Advances in Eicosanoid Biology. Science, 2001. 294(5548): p. 1871-1875.

22. Capone, M.L., et al., Pharmacodynamic of cyclooxygenase inhibitors in humans. Prostaglandins \& Other Lipid Mediators, 2007. 82(1-4): p. 85-94.

23. Rao, P.P.N., S.N. Kabir, and T. Mohamed, Nonsteroidal Anti-Inflammatory Drugs (NSAIDs): Progress in Small Molecule Drug Development. Pharmaceuticals, 2010. 3(5): p. 1530-1549.

24. Qandil, A., Prodrugs of Nonsteroidal Anti-Inflammatory Drugs (NSAIDs), More Than Meets the Eye: A Critical Review. International Journal of Molecular Sciences, 2012. 13(12): p. 17244-17274. 
25. Wolfe, M.M., D.R. Lichtenstein, and G. Singh, Gastrointestinal Toxicity of Nonsteroidal Antiinflammatory Drugs. New England Journal of Medicine, 1999. 340(24): p. 1888-1899.

26. Whelton, A., Nephrotoxicity of nonsteroidal anti-inflammatory drugs: physiologic foundations and clinical implications. The American Journal of Medicine, 1999. 106(5, Supplement 2): p. 13S-24S.

27. Pope, J.E., J.J. Anderson, and D.T. Felson, A meta-analysis of the effects of nonsteroidal anti-inflammatory drugs on blood pressure. Arch Intern Med, 1993. 153(4): p. 477-84.

28. Buchanan, W.W. and W.F. Kean, Osteoarthritis IV: Clinical therapeutic trials and treatment. Inflammopharmacology, 2002. 10(1-2): p. 79-155.

29. Hirschowitz, B.I., Nonsteroidal antiinflammatory drugs and the gastrointestinal tract. Gastroenterologist, 1994. 2(3): p. 207-23.

30. Rainsford, K.D., Profile and mechanisms of gastrointestinal and other side effects of nonsteroidal anti-inflammatory drugs (NSAIDs). Am J Med, 1999. 107(6A): p. 35S-36S.

31. Weir, M.R., Renal effects of nonselective NSAIDs and coxibs. Cleve Clin J Med, 2002. 69 Suppl 1: p. SI53-8.

32. Ahuja, M., et al., Topical Ocular Delivery of NSAIDs. The AAPS Journal, 2008. 10(2): p. 229-241.

33. Araújo, J., et al., Nanomedicines for ocular NSAIDs: safety on drug delivery. Nanomedicine: Nanotechnology, Biology and Medicine, 2009. 5(4): p. 394-401.

34. Rives, V., M. del Arco, and C. Martín, Layered double hydroxides as drug carriers and for controlled release of non-steroidal antiinflammatory drugs (NSAIDs): A review. Journal of Controlled Release, 2013. 169(1-2): p. 28-39.

35. Melarange, R., et al., Antiinflammatory and gastrointestinal effects of nabumetone or its active metabolite, 6-methoxy-2-naphthylacetic acid (6MNA). Comparative studies with indomethacin. Dig Dis Sci, 1992. 37(12): p. 1847-52.

36. Database, D.O.D.D.a.D.T. Celecoxib (DB00482 (APRD00373) ). 2013 15/07/2013 [cited 2014 30/01/2014]; Available from: http://www.drugbank.ca/drugs/DB00482.

37. Brown, K., et al., Diaryloxazole and Diarylthiazolealkanoic Acids: Two Novel Series of Non-steroidal Anti-inflammatory Agents. Nature, 1968. 219(5150): p. 164-164.

38. Rosenthale, M.E., et al., Anti-Inflammatory Activity of B-(4,5-Diphenyloxazole-2-YL) Propionic Acid (Wy-21743). Fed. Proc., 1969. 28.

39. Rosenthale, M.E., et al., Anti-inflammatory properties of 4,5-diphenyl-2oxazolepropionic acid (oxaprozin). Agents Actions, 1974. 4(3): p. 151-159.

40. Rainsford, K.D., et al., Recent pharmacodynamic and pharmacokinetic findings on oxaprozin. Inflammopharmacology, 2002. 10(3): p. 185-239.

41. Bozic, B.D., et al., Synthesis, characterization and antiproliferative activity of transition metal complexes with 3-(4,5-diphenyl-1,3-oxazol-2-yl)propanoic acid (oxaprozin). Chem Pharm Bull (Tokyo), 2012. 60(7): p. 865-9.

42. Dutta, S., S. Padhye, and V. McKee, Structural characterization and SOD activity of copper-oxaprozinate. Inorganic Chemistry Communications, 2004. 7(9): p. 1071-1074.

43. Kurowski, M. and $\mathrm{H}$. Thabe, The transsynovial distribution of oxaprozin. Agents Actions, 1989. 27(3-4): p. 458-60.

44. Administration, F.U.S.F.a.D. Daypro Alta ${ }^{\mathrm{TM}}$ (oxaprozin potassium) $600 \mathrm{mg}$ tablet. 2007 03/2007 [cited 2014 31/01/2014]; Available from: http://www.fda.gov/downloads/drugs/drugsafety/ucm088580.pdf.

45. Calvo, P., et al., Improved ocular bioavailability of indomethacin by novel ocular drug carriers. J Pharm Pharmacol, 1996. 48(11): p. 1147-52.

46. Calvo, P., J.L. Vila-Jato, and M.J. Alonso, Comparative in vitro evaluation of several colloidal systems, nanoparticles, nanocapsules, and nanoemulsions, as ocular drug carriers. J Pharm Sci, 1996. 85(5): p. 530-6.

47. Jayaprakash, S., et al., Design and Evaluation of Ketorolac Tromethamine Ocuserts. Indian Journal of Pharmaceutical Sciences, 2000. 62: p. 334-338.

48. Pignatello, R., et al., Eudragit RS100® nanosuspensions for the ophthalmic controlled delivery of ibuprofen. European Journal of Pharmaceutical Sciences, 2002. 16(1-2): p. 53-61. 
49. Pignatello, R., et al., Flurbiprofen-loaded acrylate polymer nanosuspensions for ophthalmic application. Biomaterials, 2002. 23(15): p. 3247-3255.

50. Adibkia, K., et al., Piroxicam nanoparticles for ocular delivery: physicochemical characterization and implementation in endotoxin-induced uveitis. J Drug Target, 2007. 15(6): p. 407-16.

51. Vega, E., et al., Flurbiprofen loaded biodegradable nanoparticles for ophtalmic administration. J Pharm Sci, 2006. 95(11): p. 2393-405.

52. Badawi, A., et al., Chitosan based nanocarriers for indomethacin ocular delivery. Archives of Pharmacal Research, 2008. 31(8): p. 1040-1049.

53. Contreras-García, A., et al., Stimuli-responsive networks grafted onto polypropylene for the sustained delivery of NSAIDs. Acta Biomaterialia, 2011. 7(3): p. 996-1008.

54. Čalija, B., et al., $\mathrm{pH}$-sensitive microparticles for oral drug delivery based on alginate/oligochitosan/Eudragit ${ }^{\circledR}$ L100-55 "sandwich" polyelectrolyte complex. Colloids and Surfaces B: Biointerfaces, 2013. 110(0): p. 395-402.

55. La, S.B., T. Okano, and K. Kataoka, Preparation and characterization of the micelleforming polymeric drug indomethacin-incorporated poly(ethylene oxide)-poly(Bbenzyl L-aspartate) block copolymer micelles. J Pharm Sci, 1996. 85(1): p. 85-90.

56. Giacomelli, C., V. Schmidt, and R. Borsali, Specific interactions improve the loading capacity of block copolymer micelles in aqueous media. Langmuir, 2007. 23(13): p. 6947-55.

57. Marques, J.G., et al., Synthesis and characterization of micelles as carriers of nonsteroidal anti-inflammatory drugs (NSAID) for application in breast cancer therapy. Colloids and Surfaces B: Biointerfaces, 2014. 113(0): p. 375-383.

58. Roullin, V.G., et al., Optimised NSAIDs-loaded Biocompatible Nanoparticles. NanoMicro Letters, 2010. 2(4): p. 247-255.

59. Bédouet, L., et al., Synthesis of hydrophilic intra-articular microspheres conjugated to ibuprofen and evaluation of anti-inflammatory activity on articular explants. International Journal of Pharmaceutics, 2014. 459(1-2): p. 51-61.

60. Koç, F.E. and M. Șenel, Solubility enhancement of Non-Steroidal Anti-Inflammatory Drugs (NSAIDs) using polypolypropylene oxide core PAMAM dendrimers. International Journal of Pharmaceutics, 2013. 451(1-2): p. 18-22.

61. Hasegawa, U., et al., Preparation of Well-Defined Ibuprofen Prodrug Micelles by RAFT Polymerization. Biomacromolecules, 2013. 14(9): p. 3314-3320.

62. Balasubramaniam, J., S. Kant, and J.K. Pandit, In vitro and in vivo evaluation of the Gelrite gellan gum-based ocular delivery system for indomethacin. Acta Pharm, 2003. 53(4): p. 251-61.

63. Andrade-Vivero, P., et al., Improving the loading and release of NSAIDs from PHEMA hydrogels by copolymerization with functionalized monomers. J Pharm Sci, 2007. 96(4): p. 802-13.

64. Corrente, F., et al., Novel pH-sensitive physical hydrogels of carboxymethyl scleroglucan. J Pharm Sci, 2012. 101(1): p. 256-67.

65. Saxena, A., et al., Gelucire Based In Situ Gelling Emulsions: A Potential Carrier for Sustained Stomach Specific Delivery of Gastric Irritant Drugs. BioMed Research International, 2013. 2013: p. 11.

66. Gaudio, P.D., et al., Design of alginate-based aerogel for nonsteroidal antiinflammatory drugs controlled delivery systems using prilling and supercriticalassisted drying. J Pharm Sci, 2013. 102(1): p. 185-94.

67. Sousa, C.T., et al., $\mathrm{pH}$ sensitive silica nanotubes as rationally designed vehicles for NSAIDs delivery. Colloids and Surfaces B: Biointerfaces, 2012. 94(0): p. 288-295.

68. Consola, S., et al., Design of Original Bioactive Formulations Based on SugarSurfactant/Non-steroidal Anti-inflammatory Catanionic Self-Assemblies: A New Way of Dermal Drug Delivery. Chemistry - A European Journal, 2007. 13(11): p. 30393047.

69. Ohmukai, O., Lipo-NSAID preparation. Advanced Drug Delivery Reviews, 1996. 20(23): p. 203-207.

70. Joshi, M. and V. Patravale, Formulation and evaluation of Nanostructured Lipid Carrier (NLC)-based gel of Valdecoxib. Drug Dev Ind Pharm, 2006. 32(8): p. 911-8. 
71. Joshi, M. and V. Patravale, Nanostructured lipid carrier (NLC) based gel of celecoxib. International Journal of Pharmaceutics, 2008. 346(1-2): p. 124-132.

72. Han, F., et al., Investigation of nanostructured lipid carriers for transdermal delivery of flurbiprofen. Drug Dev Ind Pharm, 2008. 34(4): p. 453-8.

73. Attama, A.A., S. Reichl, and C.C. Müller-Goymann, Diclofenac sodium delivery to the eye: In vitro evaluation of novel solid lipid nanoparticle formulation using human cornea construct. International Journal of Pharmaceutics, 2008. 355(1-2): p. 307-313.

74. Sun, K.X., et al., [Preparation of diclofenac sodium liposomes and its ocular pharmacokinetics]. Yao Xue Xue Bao, 2006. 41(11): p. 1094-8.

75. Tsukamoto, T., et al., Preparation of bromfenac-loaded liposomes modified with chitosan for ophthalmic drug delivery and evaluation of physicochemical properties and drug release profile. Asian Journal of Pharmaceutical Sciences, 2013. 8(2): p. 104-109.

76. Ulbrich, W. and A. Lamprecht, Targeted drug-delivery approaches by nanoparticulate carriers in the therapy of inflammatory diseases. J R Soc Interface, 2010. 7 Suppl 1: p. S55-66.

77. Shaji, J. and M. Lal, Nanocarriers for targeting in inflammation. Asian J Pharm Clin Res, 2013. 6: p. 3-12.

78. Hua, S., Targeting sites of inflammation: intercellular adhesion molecule-1 as a target for novel inflammatory therapies. Front Pharmacol, 2013. 4: p. 127.

79. Crielaard, B.J., et al., Drug targeting systems for inflammatory disease: one for all, all for one. J Control Release, 2012. 161(2): p. 225-34.

80. Srinath, P., S.P. Vyas, and P.V. Diwan, Preparation and pharmacodynamic evaluation of liposomes of indomethacin. Drug Dev Ind Pharm, 2000. 26(3): p. 313-21.

81. Palakurthi, S., S.P. Vyas, and P.V. Diwan, Biodisposition of PEG-coated lipid microspheres of indomethacin in arthritic rats. International Journal of Pharmaceutics, 2005. 290(1-2): p. 55-62.

82. Gerweck, L.E., S.V. Kozin, and S.J. Stocks, The $\mathrm{pH}$ partition theory predicts the accumulation and toxicity of doxorubicin in normal and low-pH-adapted cells. $\mathrm{Br} \mathrm{J}$ Cancer, 1999. 79(5-6): p. 838-42.

83. Kim, S.Y., J.C. Ha, and Y.M. Lee, Poly(ethylene oxide)-poly(propylene oxide)poly(ethylene oxide)/poly( $\epsilon$-caprolactone) (PCL) amphiphilic block copolymeric nanospheres: II. Thermo-responsive drug release behaviors. Journal of Controlled Release, 2000. 65(3): p. 345-358.

84. Moghimi, S.M., A.C. Hunter, and J.C. Murray, Long-circulating and target-specific nanoparticles: theory to practice. Pharmacol Rev, 2001. 53(2): p. 283-318.

85. Koning, G.A., R.M. Schiffelers, and G. Storm, Endothelial cells at inflammatory sites as target for therapeutic intervention. Endothelium, 2002. 9(3): p. 161-71.

86. Kinne, R.W., B. Stuhlmuller, and G.R. Burmester, Cells of the synovium in rheumatoid arthritis. Macrophages. Arthritis Res Ther, 2007. 9(6): p. 224.

87. Cox, D., M. Brennan, and N. Moran, Integrins as therapeutic targets: lessons and opportunities. Nat Rev Drug Discov, 2010. 9(10): p. 804-820.

88. Gerlag, D.M., et al., Suppression of murine collagen-induced arthritis by targeted apoptosis of synovial neovasculature. Arthritis Res, 2001. 3(6): p. 357-61.

89. Low, P.S., W.A. Henne, and D.D. Doorneweerd, Discovery and development of folicacid-based receptor targeting for imaging and therapy of cancer and inflammatory diseases. Acc Chem Res, 2008. 41(1): p. 120-9.

90. Koning, G.A., et al., Targeting of angiogenic endothelial cells at sites of inflammation by dexamethasone phosphate-containing RGD peptide liposomes inhibits experimental arthritis. Arthritis Rheum, 2006. 54(4): p. 1198-208.

91. Wang, X., et al., Differential stereospecificities and affinities of folate receptor isoforms for folate compounds and antifolates. Biochem Pharmacol, 1992. 44(9): p. 1898-901.

92. Elnakat, H. and M. Ratnam, Distribution, functionality and gene regulation of folate receptor isoforms: implications in targeted therapy. Adv Drug Deliv Rev, 2004. 56(8): p. 1067-84. 
93. Leamon, C.P. and P.S. Low, Delivery of macromolecules into living cells: a method that exploits folate receptor endocytosis. Proceedings of the National Academy of Sciences, 1991. 88(13): p. 5572-5576.

94. Chandrasekar, D., et al., The development of folate-PAMAM dendrimer conjugates for targeted delivery of anti-arthritic drugs and their pharmacokinetics and biodistribution in arthritic rats. Biomaterials, 2007. 28(3): p. 504-512.

95. Zhou, H.F., et al., Alphavbeta3-targeted nanotherapy suppresses inflammatory arthritis in mice. FASEB J, 2009. 23(9): p. 2978-85.

96. Nasongkla, N., et al., CRGD-functionalized polymer micelles for targeted doxorubicin delivery. Angew Chem Int Ed Engl, 2004. 43(46): p. 6323-7.

97. Timko, M., et al., Magnetite polymer nanospheres loaded by Indomethacin for antiinflammatory therapy. Journal of Magnetism and Magnetic Materials, 2006. 300(1): p. e191-e194.

98. Wissing, S.A., O. Kayser, and R.H. Müller, Solid lipid nanoparticles for parenteral drug delivery. Advanced Drug Delivery Reviews, 2004. 56(9): p. 1257-1272.

99. Trotta, M., et al., Insulin-Loaded SLN Prepared with the Emulsion Dilution Technique: In Vivo Tracking of Nanoparticles after Oral Administration to Rats. Journal of Dispersion Science and Technology, 2011. 32(7): p. 1041-1045.

100. Das, S. and A. Chaudhury, Recent Advances in Lipid Nanoparticle Formulations with Solid Matrix for Oral Drug Delivery. AAPS PharmSciTech, 2011. 12(1): p. 62-76.

101. Pardeike, J., A. Hommoss, and R.H. Müller, Lipid nanoparticles (SLN, NLC) in cosmetic and pharmaceutical dermal products. International Journal of Pharmaceutics, 2009. 366(1-2): p. 170-184.

102. Khan, A.A., et al., Advanced drug delivery to the lymphatic system: lipid-based nanoformulations. International Journal of Nanomedicine, 2013. 8: p. 2733-2744.

103. Severino, P., et al., Current State-of-Art and New Trends on Lipid Nanoparticles (SLN and NLC) for Oral Drug Delivery. J Drug Deliv, 2012. 2012: p. 750891.

104. Mehnert, W. and K. Mäder, Solid lipid nanoparticles: Production, characterization and applications. Advanced Drug Delivery Reviews, 2001. 47(2-3): p. 165-196.

105. Weber, S., A. Zimmer, and J. Pardeike, Solid Lipid Nanoparticles (SLN) and Nanostructured Lipid Carriers (NLC) for pulmonary application: A review of the state of the art. European Journal of Pharmaceutics and Biopharmaceutics, 2013(0).

106. Selvamuthukumar, S. and R. Velmurugan, Nanostructured lipid carriers: a potential drug carrier for cancer chemotherapy. Lipids Health Dis, 2012. 11: p. 159.

107. DeVane, L.C., Principles of pharmacokinetics and pharmacodynamics, in The American Psychiatric Publishing Textbook of Psychopharmacology, 3rd Edition, C.B.N. A.F. Schatzberg, Editor 2004, American Psychiatric Pub: Washington, DC. p. $181-200$.

108. Yun, Y., Y.W. Cho, and K. Park, Nanoparticles for oral delivery: Targeted nanoparticles with peptidic ligands for oral protein delivery. Advanced Drug Delivery Reviews, 2013. 65(6): p. 822-832.

109. Gamboa, J.M. and K.W. Leong, In vitro and in vivo models for the study of oral delivery of nanoparticles. Advanced Drug Delivery Reviews, 2013. 65(6): p. 800-810.

110. Salama, N.N., N.D. Eddington, and A. Fasano, Tight junction modulation and its relationship to drug delivery. Advanced Drug Delivery Reviews, 2006. 58(1): p. 15-28.

111. Kompella, U.B. and V.H.L. Lee, Delivery systems for penetration enhancement of peptide and protein drugs: design considerations. Advanced Drug Delivery Reviews, 2001. 46(1-3): p. 211-245.

112. Shakweh, M., G. Ponchel, and E. Fattal, Particle uptake by Peyer's patches: $a$ pathway for drug and vaccine delivery. Expert Opin Drug Deliv, 2004. 1(1): p. 141-63.

113. Russell-Jones, G.J., Carrier-mediated transport, oral drug delivery, in Encyclopedia of controlled drug delivery, E. Mathiowitz, Editor 1999, John Wiley \& Sons: New York, NY. p. 173-184.

114. Van de Graaff, K.M., Anatomy and physiology of the gastrointestinal tract. Pediatr Infect Dis, 1986. 5(1 Suppl): p. S11-6.

115. Woodley, J.F., Enzymatic barriers for GI peptide and protein delivery. Crit Rev Ther Drug Carrier Syst, 1994. 11(2-3): p. 61-95. 
116. Hamman, J.H., G.M. Enslin, and A.F. Kotze, Oral delivery of peptide drugs: barriers and developments. BioDrugs, 2005. 19(3): p. 165-77.

117. Garnett, M.C. and P. Kallinteri, Nanomedicines and nanotoxicology: some physiological principles. Occup Med (Lond), 2006. 56(5): p. 307-11.

118. Renukuntla, J., et al., Approaches for enhancing oral bioavailability of peptides and proteins. International Journal of Pharmaceutics, 2013. 447(1-2): p. 75-93.

119. Hou, D., et al., The production and characteristics of solid lipid nanoparticles (SLNs). Biomaterials, 2003. 24(10): p. 1781-1785.

120. Chan, P., et al., Synthesis and characterization of chitosan-g-poly(ethylene glycol)folate as a non-viral carrier for tumor-targeted gene delivery. Biomaterials, 2007. 28(3): p. 540-549.

121. van Steenis, J.H., et al., Preparation and characterization of folate-targeted $p E G$ coated PDMAEMA-based polyplexes. Journal of Controlled Release, 2003. 87(1-3): p. 167-176.

122. Hope, M.J., et al., Generation of multilamellar and unilamellar phospholipid vesicles. Chemistry and Physics of Lipids, 1986. 40(2-4): p. 89-107.

123. Malvern-Instruments, Zeta potential: an introduction in 30 minutes. Zetasizer Nano series technical note.

124. Malvern-Instruments, The measurement of zeta potential using an autotitrator: Effect of $\mathrm{pH}$.

125. Malvern-Instruments, Measuring Zeta Potential - Laser Doppler Electrophoresis. Zetasizer Nano Technical note.

126. Connah, M. and M. Kasuba, The use of Zeta Potential in the Pharmaceutical Industry, Malvern Instruments.

127. Woitiski, C.B., et al., Facilitated nanoscale delivery of insulin across intestinal membrane models. International Journal of Pharmaceutics, 2011. 412(1-2): p. 123131.

128. Gan, L.-S.L. and D.R. Thakker, Applications of the Caco-2 model in the design and development of orally active drugs: elucidation of biochemical and physical barriers posed by the intestinal epithelium. Advanced Drug Delivery Reviews, 1997. 23(1-3): p. 77-98.

129. Neves, A.R., et al., Novel resveratrol nanodelivery systems based on lipid nanoparticles to enhance its oral bioavailability. Int J Nanomedicine, 2013. 8: p. 177-87.

130. Maestrelli, F., et al., Improvement of oxaprozin solubility and permeability by the combined use of cyclodextrin, chitosan, and bile components. European Journal of Pharmaceutics and Biopharmaceutics, 2011. 78(3): p. 385-393. 